\title{
Silver and palladium help unveil the nature of a second r-process ${ }^{\star}, \star \star$
}

\author{
C. J. Hansen ${ }^{1,2}$, F. Primas ${ }^{1}$, H. Hartman ${ }^{3,8}$, K.-L. Kratz ${ }^{4}$, S. Wanajo ${ }^{5,6,7}$, B. Leibundgut ${ }^{1}$, K. Farouqi ${ }^{4,2}$, O. Hallmann ${ }^{4}$, \\ N. Christlieb ${ }^{2}$, and H. Nilsson ${ }^{3}$
}

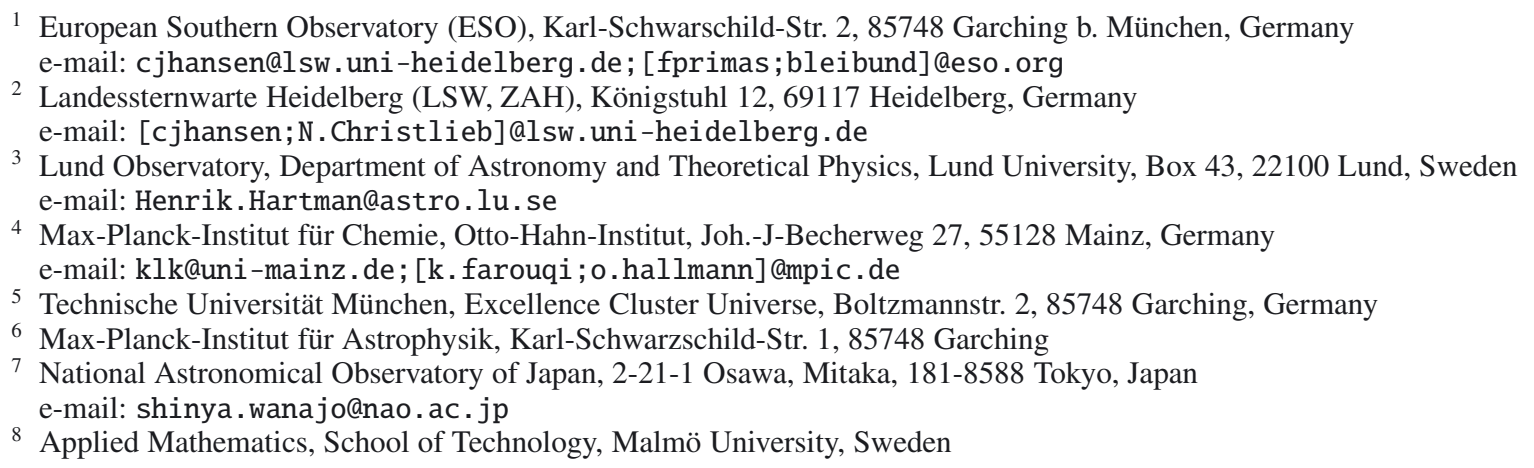

Received 14 December 2011 / Accepted 6 June 2012

\begin{abstract}
Context. The rapid neutron-capture process, which created about half of the heaviest elements in the solar system, is believed to have been unique. Many recent studies have shown that this uniqueness is not true for the formation of lighter elements, in particular those in the atomic number range $38<Z<48$. Among these, palladium (Pd) and especially silver (Ag) are expected to be key indicators of a possible second r-process, but until recently they have been studied only in a few stars. We therefore target Pd and Ag in a large sample of stars and compare these abundances to those of $\mathrm{Sr}, \mathrm{Y}, \mathrm{Zr}, \mathrm{Ba}$, and Eu produced by the slow (s-) and rapid (r-) neutron-capture processes. Hereby we investigate the nature of the formation process of $\mathrm{Ag}$ and $\mathrm{Pd}$.

Aims. We study the abundances of seven elements ( $\mathrm{Sr}, \mathrm{Y}, \mathrm{Zr}, \mathrm{Pd}, \mathrm{Ag}, \mathrm{Ba}$, and $\mathrm{Eu}$ ) to gain insight into the formation process of the elements and explore in depth the nature of the second r-process.

Methods. By adopting a homogeneous one-dimensional local thermodynamic equilibrium (1D LTE) analysis of 71 stars, we derive stellar abundances using the spectral synthesis code MOOG, and the MARCS model atmospheres. We calculate abundance ratio trends and compare the derived abundances to site-dependent yield predictions (low-mass O-Ne-Mg core-collapse supernovae and parametrised high-entropy winds), to extract characteristics of the second r-process.

Results. The seven elements are tracers of different (neutron-capture) processes, which in turn allows us to constrain the formation process(es) of Pd and Ag. The abundance ratios of the heavy elements are found to be correlated and anti-correlated. These trends lead to clear indications that a second/weak r-process, is responsible for the formation of Pd and $\mathrm{Ag}$. On the basis of the comparison to the model predictions, we find that the conditions under which this process takes place differ from those for the main r-process in needing lower neutron number densities, lower neutron-to-seed ratios, and lower entropies, and/or higher electron abundances. Conclusions. Our analysis confirms that Pd and $\mathrm{Ag}$ form via a rapid neutron-capture process that differs from the main r-process, the main and weak s-processes, and charged particle freeze-outs. We find that this process is efficiently working down to the lowest metallicity sampled by our analysis $([\mathrm{Fe} / \mathrm{H}]=-3.3)$. Our results may indicate that a combination of these explosive sites is needed to explain the variety in the observationally derived abundance patterns.
\end{abstract}

Key words. stars: abundances - stars: Population II - supernovae: general - Galaxy: halo - atomic data

\section{Introduction}

The heavy elements beyond the iron-peak are not created in the same way as the lighter elements, many of which form via hydrostatic core or shell burning in the star. These elements are generally created by various neutron-capture processes taking

\footnotetext{
* Based on observations made with the ESO Very Large Telescope at Paranal Observatory, Chile (ID 65.L-0507(A), 67.D-0439(A), 68.B0475(A), 68.D-0094(A), 71.B-0529(A); P.I. F. Primas).

$\star \star$ Appendices are available in electronic form at

http://www . aanda.org
}

place as either the result of mixing in very evolved stars or explosions ${ }^{1}$.

Previous studies have shown that the slow neutron-capture (s-) process can be classified into two sub-processes, namely a weak s-process creating the lighter of the s-process isotopes (Prantzos et al. 1990; Heil et al. 2009; Pignatari et al. 2010), and a main s-process creating heavy isotopes, such as those of barium (Käppeler et al. 1989; Busso et al. 1999; Gallino et al. 2006; Sneden et al. 2008). The sites of the rapid neutron-capture

${ }^{1}$ We disregard proton processes here. 
(r-) processes remain unclear, and the exact conditions under which they operate continue to be investigated. Since the time of Burbidge et al. (1957), it has been evident that an explosive environment is needed to provide the proper conditions for an r-process to happen. After several attempts to make site-dependent predictions of the neutron-capture processes, Kratz et al. (1993) provided a site-independent approach using the so-called waiting point approximation, which is based on the best available nuclear physics to shed light on the r-process. Nevertheless, the conditions are still poorly constrained. A number of sites have been suggested: neutron star mergers (Freiburghaus et al. 1999b; Goriely et al. 2011a,b; Wanajo \& Janka 2012), massive core-collapse supernovae (SNe) (Wasserburg \& Qian 2000; Argast et al. 2004), neutrino-driven winds from core-collapse SNe (Duncan et al. 1986; Meyer 1993; Takahashi et al. 1994; Woosley et al. 1994; Freiburghaus et al. 1999a; Wanajo et al. 2001; Farouqi et al. 2009, 2010; Arcones \& Montes 2011), low-mass SNe from collapsing O-Ne-Mg cores (Wanajo et al. 2003; or iron cores Sumiyoshi et al. 2001). However, no consensus on the formation site has been reached.

Observationally, the discovery of r-process-rich stars which contain a factor of 20-100 more heavy elements than normal Population II halo stars; (see Hill et al. 2002; Sneden et al. 2003; Christlieb et al. 2004; Barklem et al. 2005; Frebel et al. 2007; Hayek et al. 2009; Aoki et al. 2010; Cowan et al. 2011), has offered an important opportunity to study in greater detail the r-process and its characteristics. By comparing light to heavy neutron-capture elements (i.e. $38<Z<50$ vs. $Z>56$ ), some of these studies (Sneden et al. 2000; Westin et al. 2000; Johnson \& Bolte 2002; Christlieb et al. 2004; Honda et al. 2004; Barklem et al. 2005; Honda et al. 2006, 2007; François et al. 2007; Sneden et al. 2008; Kratz et al. 2008b; Roederer et al. 2010) have revealed a departure of the "light" neutron capture elements from the main solar-scaled r-process distribution curve, which was interpreted as an indication of an extra process. This suggests that the r-process may also split into two sub-channels, namely a "weak" and main one (Cowan et al. 1991; Wanajo \& Ishimaru 2006; Ott \& Kratz 2008), which are responsible for the production of the lighter and heavier r-process isotopes, respectively. The nomenclature is used to match the s-process (Käppeler et al. 1989).

The "weak" r-process has received a lot of recent attention, but is still poorly constrained despite the many attempts that have been made to understand this process. Some of the proposed processes are the lighter element primary process (LEPP, Travaglio et al. 2004; Arcones \& Montes 2011), the weak r-process (Kratz et al. 2007; Montes et al. 2007; Farouqi et al. 2009; Wanajo et al. 2011), the $v$ p-process (Fröhlich et al. 2006), and several more processes and comparisons, which can be found in Cowan et al. (2001), Qian \& Wasserburg (2001), and Sneden et al. (2003). These processes can be considered when attempting to explain the abundances of the lighter heavy elements, which have been found to deviate from the solar-scaled r-process pattern ${ }^{2}$. Palladium and silver are among these lighter heavy elements. Silver was studied for the first time by Crawford et al. (1998) more than a decade ago in a small sample of metal-poor stars. They applied a different hyperfine split oscillator strength from the one we adopt here, which together with the higher solar Ag abundance helps us to explain the low silver abundances they derive. A few years later, Johnson \& Bolte (2002) studied both $\mathrm{Pd}$ and $\mathrm{Ag}$ in a sample that is the only other relatively large sample where both Pd and Ag were analysed. Hence, we

\footnotetext{
${ }^{2}$ Solar-scaled r-process abundance: $N_{\mathrm{r}}=N_{\odot}-N_{\mathrm{s}}$.
}

compare our results to theirs. Hansen \& Primas (2011) presented the first results of an analysis of $\mathrm{Ag}$ and $\mathrm{Pd}$ in a large sample (55 stars) that demonstrated the need for an extra production channel. Here, we extend the study to the entire sample (71 stars) and compare our derived $\mathrm{Ag}$ and $\mathrm{Pd}$ abundances to those of five other heavy elements, namely Sr, Y, Zr, Ba, and Eu. We furthermore wish to explore the nature of the second r-process in depth by investigating the trends of two particular tracer elements, palladium and silver. We characterise and constrain the fundamental parameters of the formation of these elements by means of a detailed comparison to yield predictions from several of the above-mentioned astrophysical sites and objects. Silver and palladium are important for two reasons. First, silver is predicted to be a good tracer of the weak r-process since nearly $80 \%$ of its solar system abundance is predicted (Arlandini et al. 1999; Sneden et al. 2008; Lodders et al. 2009) to have come from the r-process, and more than $71 \%$ of the r-process is estimated to originate from the weak r-process (Kratz et al. 2008b; Farouqi et al. 2009; Roederer et al. 2010). For comparison, only 54\% of palladium is created by the r-process (Arlandini et al. 1999). Second, these two elements had only been studied in a small number of stars $(<20)$ until Hansen \& Primas (2011), whereas many other neutron-capture elements such as Ba have been studied in hundreds of stars (e.g. Reddy et al. 2006; Barklem et al. 2005; François et al. 2007; Roederer 2009). A study of palladium and silver provides astrophysical information on a poorly studied part of the periodic table.

The paper is organised as follows: Sect. 2 describes the observations and data, Sect. 3 outlines the stellar parameter determination, Sect. 4 presents new atomic data and calibration of the line list, Sect. 5 presents the abundance analysis, and Sects. 6 and 7 provide the results and discussions of our abundance and model comparisons, respectively. Finally, our conclusions can be found in Sect. 8.

\section{Observations and data reduction}

Our sample consists of a mixture of dwarf and giant stars, which were observed at high resolution $(R>40000)$. The dwarfs were observed in the years 2000-2002 with the UltraViolet Echelle Spectrograph at the Very Large Telescope, UVES/VLT, Dekker et al. (2000) for a project targeting beryllium, which requires high signal-to-noise $(\mathrm{S} / \mathrm{N})$ data of the near-ultraviolet (near-UV) particularly the Be doublet at $313 \mathrm{~nm}$ (Primas 2010). Similarly high quality data are also needed to detect silver and palladium $(328.6,338.3 \mathrm{~nm}$, and $340.4 \mathrm{~nm}$, respectively). The spectra cover the wavelength ranges $\sim 305-680 \mathrm{~nm}$ (in some cases up to $1000 \mathrm{~nm}$ ), including the wavelength gaps between the CCD detectors. All of our UVES spectra have a $S / N>100$ per pixel at $320 \mathrm{~nm}$. The dwarf spectra were reduced with the UVES pipeline (v. 4.3.0). The pipeline performs a standard echelle spectrum data reduction. It starts with bias subtraction, removes bad pixels due to e.g. cosmic ray hits, and locates the orders. Then a background subtraction is followed by flat field division, order extraction, and wavelength calibration, and finally the orders are merged. We tested the quality of the data products against a manual data reduction carried out in $\mathrm{IRAF}^{3}$ because previous versions of the pipelines had problems with the order merging. However, this pipeline performs very well and the reduced data were compatible with manually reduced data. Finally,

3 IRAF is distributed by the National Optical Astronomy Observatory, which is operated by the Association of Universities of Research in Astronomy, Inc., under contract with the National Science Foundation. 
the reduced spectra were radial velocity corrected/shifted via cross correlation, coadded, and had their continua normalised (in IRAF).

The spectra of the giants were instead extracted from public data archives of the VLT and the Keck telescopes. In both cases, the spectra were observed with the high-resolution spectrographs available on both sites, i.e. UVES (Dekker et al. 2000) on the VLT and HIgh REsolution Spectrometer HIRES (Vogt et al. 1994) on Keck. The wavelength coverage of HIRES spans $300-1000 \mathrm{~nm}$, which is very similar to the wavelength range of UVES but might have gaps above $620 \mathrm{~nm}$. Only spectra of high and comparable (to the dwarfs') quality were added to the sample. The giant spectra extracted from the respective archives had already been reduced, and were carefully inspected, radial velocity shifted, coadded, and continua normalised in IRAF.

\section{Sample}

The final stellar sample consists of 42 dwarf and 29 giant field stars, belonging to the Galactic halo, the thick, and the thin disks. The sample spans a broad parameter range exceeding $2000 \mathrm{~K}$ in temperature, 4 dex in gravity, and 2.5 dex in metallicity. Such a sample composition allows us to explore the chemical evolution of the Galaxy, as well as test the different chemical signatures of different stellar evolutionary stages. This in turn can shed light on the importance of mixing and non-local thermodynamic equilibrium (NLTE) effects.

Our sample includes some of the most well-known r-process enhanced giant stars including CS 31082-001 (Hill et al. 2002), which we compare to CS 22892-052 (Sneden et al. 2003), and BD +173248 (Cowan et al. 2011). We note that only one r-process enhanced metal-poor dwarf star has been found and observed so far (Aoki et al. 2010), which is not included here. Furthermore, silver lines can be detected in giants of all the metallicities studied here, but can only be detected in dwarfs with $[\mathrm{Fe} / \mathrm{H}]>\sim-2.0$. This may introduce a small sample bias towards metal-poor r-process enhanced giants. No carbonenhanced stars were included in our sample.

\section{Stellar parameters}

We followed different methods to determine the optimal set of stellar parameters. With such a large sample, we faced some difficulties in applying the same method to the determination of the stellar parameters for the entire data-set. The effective temperature of most of our stars was derived from colour- $T_{\text {eff }}$ calibrations to which we applied the necessary band-filter and colour corrections. In this respect, we tested several different colour calibrations from Alonso et al. (1996), Alonso et al. (1999), Ramírez \& Meléndez (2005), Masana et al. (2006), Önehag et al. (2009), and Casagrande et al. (2010), who make use of both $(V-K)$ and $(b-y)$ colour indices. In the end, we chose the calibration of Alonso et al. $(1996,1999)$ because these lead to temperature predictions that generally fall in the middle of the range shown in Fig. 1. The temperature has a large influence on the derived stellar abundances. Hence, we wished to avoid systematic effects in the abundances by over-/under-estimating the temperature, and therefore selected an intermediate temperature scale. The photometry was from 2 MASS $(K)$ and Johnson $V$ (the $V-K$ was taken from Cutri et al. 2003) and the parallax was taken from the Hipparcos catalogue (Perryman et al. 1997).

Our final effective temperatures are based only on the $(V-K)$ colour index. Among the indices, we considered it to be the most

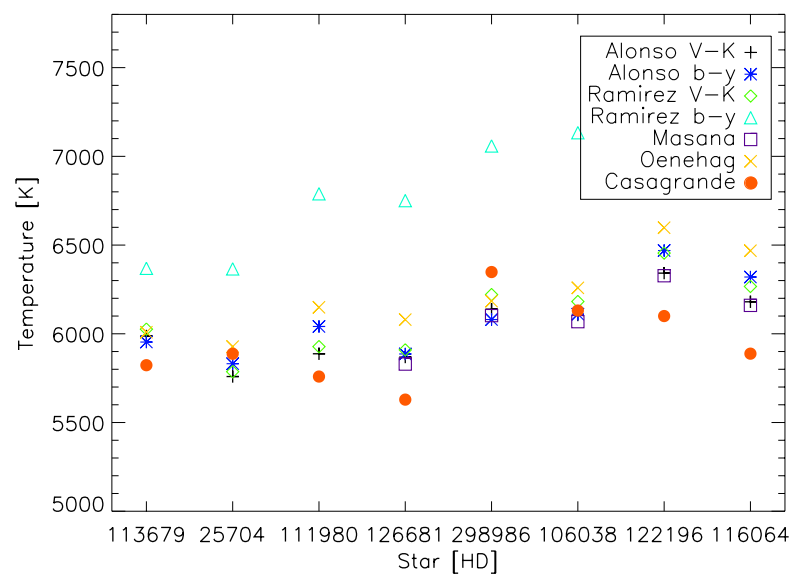

Fig. 1. Effective temperatures derived for eight stars (of different metallicity, from higher to lower as one moves from left to right along the $x$-axis) with seven different colour- $T_{\text {eff }}$ calibrations (see figure legend).

metallicity-independent one (Alonso et al. 1999), since infrared magnitudes are less affected by reddening ( $K$ is the only infra-red magnitude that is available for all our sample stars). Additionally, the temperatures derived for the dwarfs based on this colour are in good agreement with those determined via $\mathrm{H}_{\beta}$ line fitting (Nissen et al. 2007). We note, however, that the $(b-y)$ colour tends to predict slightly higher temperature values than $(V-K)$.

The reddening corrections, $E(B-V)$, were mostly derived from the Schlegel dust maps ${ }^{4}$ (Schlegel et al. 1998) and corrected according to Bonifacio et al. (2000) if they exceeded $0.1 \mathrm{mag}$. For a few stars, we took the corresponding $E(B-V)$ values from the literature (Nissen et al. 2002, 2004, 2007). We applied the formula of Alonso et al. (1996) of $E(V-K)=$ $2.72 E(B-V)$, which corresponds to the average of those of Ramírez \& Meléndez (2005), Kinman \& Castelli (2002), and Nissen et al. (2002). A filter conversion of -0.04 from Bessell (2005, 2MASS to Johnson) transformed the $K$ magnitudes from the 2MASS to the Johnson system, and brought both magnitudes to the Johnson scale leading to:

$V-K_{0, \mathrm{Johnson}}=V_{\mathrm{Johnson}}-K_{2 \mathrm{MASS}}-0.04-2.72 E(B-V)$.

Having both magnitudes on the Johnson scale, we converted $V-K$ from Johnson to TCS (Observatorio del Teide), which can be done by applying the following relation from Alonso et al. (1994)

$(V-K)_{\mathrm{TCS}}=0.05+0.994(V-K)_{\mathrm{Johnson}}$.

This last part of the filter conversion - Johnson to TCS - corresponds on average to +0.04 mag. We keep all transformations for the sake of accuracy.

In the case of stars (typically, from the disk) found to have unrealistically large $E(B-V)$ values we decided to derive their temperatures spectroscopically. The gravity was calculated from Hipparcos parallaxes by applying the classical formula

$\log \frac{g}{g_{\odot}}=\log \frac{M}{M_{\odot}}+4 \log \frac{T_{\text {eff }}}{T_{\text {eff } \odot}}-4 V_{0}+0.4 B C+2 \log \pi+0.12$,

where $M$ is the mass, $V_{0}$ is the dereddened apparent magnitude, $B C$ is the bolometric correction ${ }^{5}$, and $\pi$ is the parallax. Stellar

\footnotetext{
${ }^{4}$ http://spider.ipac.caltech.edu/staff/jarrett/irsa/ dust.html

5 Adopted from Nissen et al. (1997).
} 
masses were taken from the literature (Nissen et al. 2002, 2004, 2007). On the basis of Alonso et al. (1995), we calculated the $\mathrm{BC}$ for each of our stars. For the few stars for which no parallax was available, we constrained their gravities by enforcing ionisation equilibrium between $\mathrm{Fe}$ I and $\mathrm{Fe} \mathrm{II}^{6}$. In general, the Fe I and II abundances are in good agreement, although we note that for stars labelled with an "a" or "c" in Table B.1 the parallax was neglected (either owing to their large uncertainties or wideranging Fe I and II abundances when the gravity was derived from the parallax). The metallicity was derived from Fe I equivalent widths (EWs) and is in good agreement with previous studies. The microturbulence was determined by requiring that all Fe I lines yield the same abundance regardless of their EW. The final values and adopted methods are presented in Appendix B.

\subsection{Error estimates for the stellar parameters}

The largest source of uncertainty in estimating the temperature is the dereddening of the colour indices, e.g. applying overestimated reddening values from the Schlegel dust maps to stars close to the Galactic plane. These can easily translate into uncertainties of several hundred Kelvin in the derived temperature. Disregarding these extreme cases, we found that the general uncertainty in the reddening values is usually $0.05 \mathrm{mag}$. Combining these values of 0.05 mag with the uncertainty due to the Johnson2MASS transformation led to typical uncertainties of the order of $100-150 \mathrm{~K}$. A slight magnitude-temperature offset was found between giants and dwarfs owing to the stronger colour dependence of the dwarfs' temperature compared to that of the giants. Similar uncertainties were found for the excitation temperatures.

Since all stellar parameters to some extent are interdependent, we also tested the influence of gravity and metallicity on the temperature. For instance, an uncertainty of \pm 0.15 dex in metallicity has a negligible effect on the temperature (the uncertainty is usually a few Kelvin). An uncertainty of \pm 0.2 dex in gravity causes an uncertainty in the temperature of $< \pm 1-10 \mathrm{~K}$. Finally, the microturbulent velocity is found to have a negligible impact on the temperature.

The main uncertainty in the gravity comes from the uncertainty in the parallax, which is on average $\pm 1.0^{\prime \prime}$ (Perryman et al. 1997). This translates into $\lesssim 0.2$ dex in $\log g$. A change of $\pm 100 \mathrm{~K}$ in temperature only causes a gravity uncertainty of \pm 0.04 dex. By altering the gravity by \pm 0.25 dex, the Fe II abundance is affected by \pm 0.15 dex, whereas the Fe I abundance remains basically the same.

The metallicity is based on EW measurements for which Fe I and Fe II lines provided consistent results, usually agreeing to within 0.1 dex. Since our derived metallicities closely match those found in the literature (most of our stars are well-studied Galactic halo and disk stars), our typical adopted uncertainty in the metallicity is \pm 0.1 dex ( \pm 0.15 dex in only a few cases).

For the microturbulence velocity, we estimated uncertainties of the order of $0.15 \mathrm{~km} \mathrm{~s}^{-1}$, stemming from the uncertainty in the $[\mathrm{Fe} / \mathrm{H}]$ and the uncertainty in the Fe EW measurements (which is of the order of $\pm 2 \mathrm{~m} \AA$, as tested via repeated independent measurements).

\footnotetext{
${ }^{6}$ In total, we have 13 stars for which no reliable information on either their $(V-K)$ colour, parallax, or reddening correction, $E(B-V)$, is available. Hence, we resort to spectroscopically derived stellar parameters, i.e. excitation temperatures and gravities constrained via $\mathrm{Fe} \mathrm{I} / \mathrm{Fe}$ II ionisation equilibrium (see also letter " $a$ " and "c" in Appendix B).
}

\section{Atomic data and line lists}

This section is divided into two. The first part presents the newly calculated $\log g f$ values of silver, and the second part describes the adjustments and calibrations carried out on the line lists. We first note that similar calculations are not necessary for palladium. This element has six naturally occurring stable isotopes $(102,104,105,106,108,110)$, of which only four are accessible to the r-process. ${ }^{105} \mathrm{Pd}$ is the only odd-mass isotope with nuclear spin 5/2 for which hyperfine splitting exists. The effect on the oscillator strength is, however, minor, since this isotope only contributes $22.33 \%$ of its solar elemental abundance. Hence, we continue focusing only on the hyperfine structure (hfs) of silver.

\subsection{Atomic data}

This section focuses on the derivation of the hfs of the resonance lines in Ag I.

Silver has two stable isotopes with mass numbers 107 and 109 , respectively. The nuclear spin is $I=1 / 2$ for each of the isotopes. As a consequence, each fine structure level is split into two hyperfine levels. The resonance lines in Ag I connect the lower $5 \mathrm{~s}$ level to the $5 \mathrm{p}$ levels.

The isotopic and hyperfine structures commonly used in abundance studies of the Ag resonance lines are those given in Ross \& Aller (1972). They derived $\log g f$ values for the different hyperfine and isotopic components using the experimental studies of the relative hfs pattern conducted by Jackson \& Kuhn (1937) and Crawford et al. (1949). These are intensity measurements of different components studied by interferometric experiments. Ross \& Aller (1972) label four components, i.e. two hyperfine components for each isotope. The expected number of components are three for each of the isotopes 107 and 109 (see Tables 1 and A.1). The uncertainty in the old intensity measurements resulted in a misinterpretation and misidentification of the components.

We derive new hyperfine transition components based on several experimental measurements of the hfs from more recent studies, using the theory of the addition of angular momenta to derive the hyperfine components. We also derive experimental oscillator strengths, $\log g f$ values, for the different components. The transition energies are derived from unresolved Fourier transform spectroscopy (FTS) measurements.

\section{Hyperfine structure components}

The splitting due to the hfs of a level is given by

$\Delta E_{\mathrm{hfs}}=\frac{1}{2} A_{\mathrm{hfs}}[F(F+1)-J(J+1)-I(I+1)]$,

where $A_{\mathrm{hfs}}$ is the hyperfine magnetic dipole constant. For nuclei with larger spin, the electric quadrupole moment can be significant, but for nuclei of $\operatorname{spin} I=1 / 2$, as for $\mathrm{Ag}$, only the magnetic dipole is non-zero (Cowan 1981). The quantum numbers $I, J$, and $F$ are those related to the nuclear spin, total angular momentum of the electrons, and the total angular momentum with the nuclear spin taken into account, respectively. This expression assumes that the coupling among the electrons, resulting in a total angular momentum $J$, is much stronger than the coupling to the nuclear angular momentum $I$. The interaction between $I$ and $\boldsymbol{J}$ are coupled to a moment $\boldsymbol{F}$.

\footnotetext{
${ }^{7}$ http://www. tracesciences.com/pd.htm
} 
C. J. Hansen et al.: Silver and palladium help unveil the nature of a second r-process

Table 1. Model parameters for the silver resonance lines, assuming an isotopic ratio of $51.84 \%$ for isotope 107 and $48.16 \%$ for isotope 109 .

\begin{tabular}{lccccc}
\hline \hline Isotope & Lower level & Upper level & Flow-Fup & $\begin{array}{c}\lambda_{\text {air }} \\
{[\AA]}\end{array}$ & Reduced log gf \\
\hline 107 & ${ }^{2} \mathrm{~S}_{1 / 2}$ & ${ }^{2} \mathrm{P}_{1 / 2}$ & $0-1$ & 3382.891 & -1.221 \\
107 & ${ }^{2} \mathrm{~S}_{1 / 2}$ & ${ }^{2} \mathrm{P}_{1 / 2}$ & $1-0$ & 3382.884 & -1.221 \\
107 & ${ }^{2} \mathrm{~S}_{1 / 2}$ & ${ }^{2} \mathrm{P}_{1 / 2}$ & $1-1$ & 3382.885 & -0.920 \\
109 & ${ }^{2} \mathrm{~S}_{1 / 2}$ & ${ }^{2} \mathrm{P}_{1 / 2}$ & $0-1$ & 3382.894 & -1.253 \\
109 & ${ }^{2} \mathrm{~S}_{1 / 2}$ & ${ }^{2} \mathrm{P}_{1 / 2}$ & $1-0$ & 3382.886 & -1.253 \\
109 & ${ }^{2} \mathrm{~S}_{1 / 2}$ & ${ }^{2} \mathrm{P}_{1 / 2}$ & $1-1$ & 3382.887 & -0.952 \\
\hline & & & & total log gf & -0.334 \\
107 & ${ }^{2} \mathrm{~S}_{1 / 2}$ & ${ }^{2} \mathrm{P}_{3 / 2}$ & $0-1$ & 3280.684 & -0.909 \\
107 & ${ }^{2} \mathrm{~S}_{1 / 2}$ & ${ }^{2} \mathrm{P}_{3 / 2}$ & $1-1$ & 3280.678 & -1.210 \\
107 & ${ }^{2} \mathrm{~S}_{1 / 2}$ & ${ }^{2} \mathrm{P}_{3 / 2}$ & $1-2$ & 3280.678 & -0.511 \\
109 & ${ }^{2} \mathrm{~S}_{1 / 2}$ & ${ }^{2} \mathrm{P}_{3 / 2}$ & $0-1$ & 3280.686 & -0.941 \\
109 & ${ }^{2} \mathrm{~S}_{1 / 2}$ & ${ }^{2} \mathrm{P}_{3 / 2}$ & $1-1$ & 3280.679 & -1.242 \\
109 & ${ }^{2} \mathrm{~S}_{1 / 2}$ & ${ }^{2} \mathrm{P}_{3 / 2}$ & $1-2$ & 3280.680 & -0.543 \\
\hline \multicolumn{5}{r}{} \\
\hline
\end{tabular}

Notes. The transition strengths (reduced $\log g f$ ) given are not the true $\log g f$, but were adjusted for the natural isotopic ratio. For studies treating the isotopes using individual abundances, the data in Table A.1 should be used.

The energy splitting for a given level can thus be derived from the hyperfine constant. The hyperfine constants $A_{\mathrm{hfs}}$ for the $5 \mathrm{p}$ levels were measured by Carlsson et al. (1990) by observing quantum beats. The splitting of the $5 \mathrm{~s}$ level is an order of magnitude larger and was measured by Dahmen \& Penselin (1967). From the energy splittings, the relative wavelengths for the transitions can be derived.

The intensity ratios for the transitions between the different hyperfine components can be derived using the expressions for the addition of angular momenta (e.g. Cowan 1981), where the decay in each channel is proportional to

$A \propto(2 F+1)\left(2 F^{\prime}+1\right)\left\{\begin{array}{ccc}J & I & F \\ F^{\prime} & 1 & J^{\prime}\end{array}\right\}^{2}$,

and the prime is for the lower level. From the hyperfine constants of the $5 \mathrm{~s}$ and $5 \mathrm{p}$ levels, the hyperfine pattern with relative intensities and splitting can be derived. This gives the relative intensities and positions of the hyperfine components for one isotope, but not the relative shift between the isotopes.

We used the interferometric observations of Jackson \& Kuhn (1937) to derive the shift between the two isotopes. The resolved components in their measurements were, with the aid of the predicted hfs for each isotope, used to derive the isotopic shift. We used the resolved components $\left(F_{\mathrm{u}}-F_{1}\right.$ : $\left.1-0\right)$ to establish the isotopic shift, which are $0.026 \mathrm{~cm}^{-1}$ and $0.022 \mathrm{~cm}^{-1}$ for the $5 \mathrm{~s}^{2} \mathrm{~S}_{1 / 2}-5 \mathrm{p}{ }^{2} \mathrm{P}_{1 / 2}$ and $5 \mathrm{~s}^{2} \mathrm{~S}_{1 / 2}-5 \mathrm{p}{ }^{2} \mathrm{P}_{3 / 2}$, respectively. The resulting structure for the two resonance lines are shown in Fig. 2.

The absolute wavelengths of the different components were derived from the centre of gravity of the resonance lines measured by Pickering \& Zilio (2001), who used a hollow cathode discharge and Fourier Transform Spectrometer. The hyperfine and isotopic structure are too small to be resolved in the Doppler broadened line profiles.

\section{Transition strengths}

The derivation of the line structure due to isotopic and hyperfine structure above give the relative intensities. To use the transitions for quantitative studies, we need the absolute values, i.e. the oscillator strengths $(\log g f)$, which can be derived from the radiative lifetime of the upper levels.
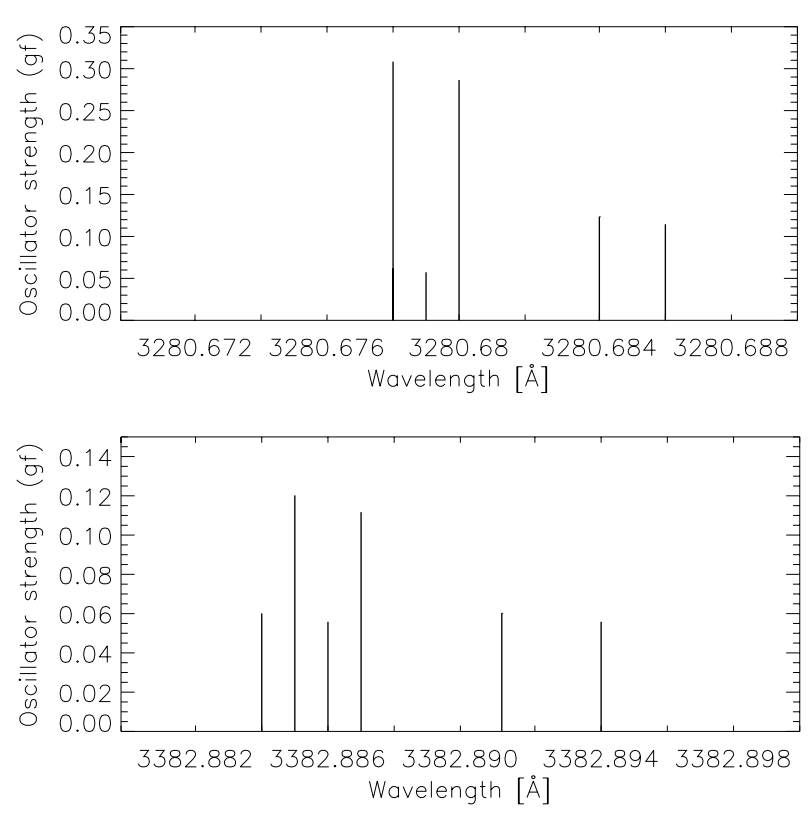

Fig. 2. Hyperfine and isotopic structure of the resonance lines of Ag, calculated using a natural isotopic abundance.

The lifetimes for the upper levels of the resonance transitions, $5 p^{2} \mathrm{P}_{1 / 2,3 / 2}$ were measured using a laser induced fluorescence technique by Carlsson et al. (1990). Since there is only one decay channel per level, the transition rates $(A)$ are simply given by the inverse of the lifetime as $A=1 / \tau$.

The absolute transition rates can, combined with the relative intensities of the hyperfine components for a given fine structure transition as discussed above, give the $\log g f$ value for the individual hyperfine components according to

$g f=1.499 \times 10^{-14} \lambda^{2} g A$,

where $\lambda$ is given in $\mathrm{nm}$ and $g$ is the statistical weight. These are reported in Table A.1.

The hyperfine and isotopic structures of $\mathrm{Ag}$ is rather small and cannot be resolved in the stellar spectrum. The contribution from the different isotopes can thus rarely be measured. To 


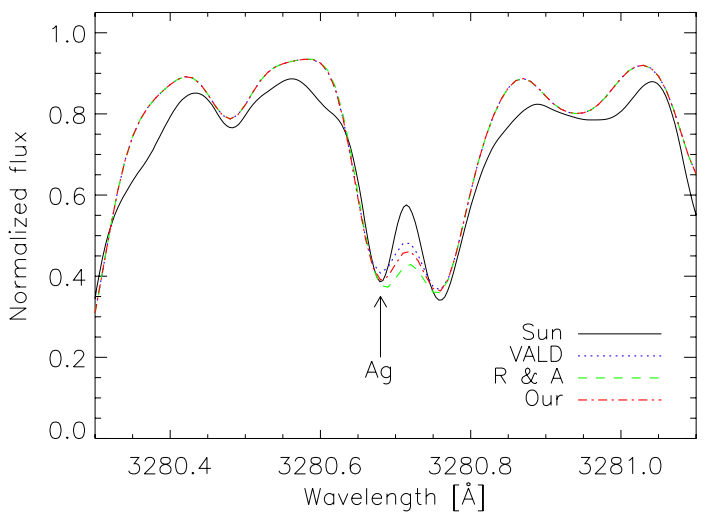

Fig. 3. The Kitt Peak solar spectrum with spectrum synthesis computed with different line lists overplotted: VALD's $\log g f$ without hfs (dotted blue line); our most recent $\log g f$ values (dash-dotted red line); and the old hfs (only two levels) values from Ross \& Aller (1972) (R \& A, dashed green line).

handle the different isotopes in the stellar spectrum, it is usually assumed that the isotope ratio is the same as the natural abundance: $51.84 \%$ for isotope 107 and $48.16 \%$ for isotope 109 . It is convenient to derive the contribution to the $\mathrm{Ag}$ absorption lines from the different isotopes, normalising to the isotopic ratio. The line parameters for a natural abundance mix of isotopes is given in Table 1. It should be noted, however, that the true $\log g f$ is an atomic parameter for each isotope, which is independent of the isotopic ratio, and the values in Table 1 are to be used only with a fixed isotopic ratio and for a total $\mathrm{Ag}$ abundance. For a strict treatment of the individual abundances for the two isotopes, the values in Table A.1 should be used.

Figure 3 shows the effect of including hyperfine splitting with zero, two or three hfs levels. If we had adopted the $\log g f$ value available from VALD (the Vienna Atomic Line Database ${ }^{8}$, Kupka 2000) without hfs, all the Ag abundances would have been overestimated. This effect is even more pronounced in the cool metal-rich stars, where the silver lines are stronger. In dwarf stars such as the Sun, the new hfs predicted $\log g f$ values can lead to a difference of $\lesssim+0.2$ dex in the derived silver abundances, compared to the results based on Ross \& Aller (1972) values (see Fig. 3). Hence, neglecting hfs would lead to overestimated silver abundances.

\section{Silver isotopes}

Based on measurements of the visual and near-infrared Ag I and II lines (Elbel \& Fischer 1962), silver is predicted to show a relatively small isotopic shift, which would barely affect the spectral line at our spectral resolution. We carried out a test for the nearUV lines with natural isotopic abundance (which is $~ 48 / 52 \%$ for $109 / 107 \mathrm{Ag}$ ) and compared this to two other test cases with ratios of $25 / 75 \%$ and $1 / 99 \%$ for the $109 / 107 \mathrm{Ag}$ isotopes, respectively. The actual change in the synthetic spectrum was less than the width of the plotted line. Hence, the change in isotopic fraction could be seen in neither our high quality spectra nor the high-resolution Kitt Peak spectrum of the Sun.

\subsection{Line list}

We now focus on the silver and palladium lines and their atomic data, since these elements are the ones that have been studied

\footnotetext{
${ }^{8}$ http://vald.astro.univie.ac.at/ vald/php/vald.php
}

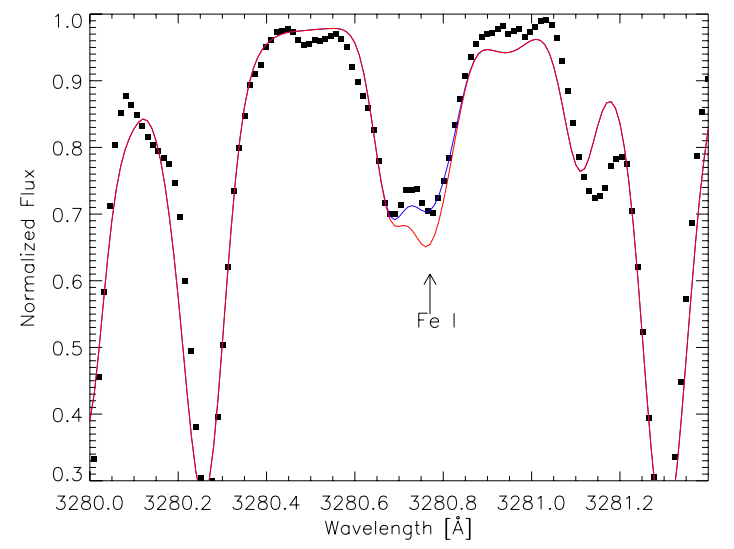

Fig. 4. Effect of a wrong $\log g f$ of the blending Fe line (marked by an arrow) shown for HD 121004. The $\log g f$ of Fe I transition (red line) over-predicts the Fe line strength, resulting in an underestimation of the $\mathrm{Ag}$ abundance. The synthesis using our adjusted $\mathrm{Fe} \mathrm{I} \log g f$ value is shown in blue.

the least. The line list for the $\mathrm{Sr}, \mathrm{Y}, \mathrm{Zr}, \mathrm{Ba}$, and Eu lines is not reported here. They include the most commonly used transitions of these elements, and can be found in Table A.3.

In general, all atomic data were taken from VALD (Kupka 2000), and we cross-checked excitation potentials and oscillator strengths $\left(\log g f\right.$ ) against the NIST $^{9}$ (National Institute of Standards and Technology) compilation and recent literature, in order to get the most up-to-date line list and best possible abundances.

From VALD, we excluded all weak lines ${ }^{10}$, i.e. lines with excitation potential higher than $4 \mathrm{eV}$ and $\log g f$ values smaller than -4 dex. These weak lines have no significant influence on the continuum, thus do not affect the derivation of the $\mathrm{Ag}$ abundances. We note that the same approach was followed by Johnson \& Bolte (2002), which we adopted to be able to make a direct comparison to their (the only other) large available sample.

The silver lines are situated at $3280.7 \AA$ and $3382.9 \AA$ and the palladium line used in this study falls at $3404.58 \AA$. In this near-UV region, the molecular lines $(\mathrm{OH}$ and especially $\mathrm{NH}$ ) make a significant contribution to the spectrum, and all molecular line information was taken from Kurucz's database ${ }^{11}$. In addition, we note that this wavelength region suffers from unidentified transitions. Therefore, one predicted line from Kurucz - the $3382.96 \AA$, Fe I line - was included in our final list in order to produce a satisfactory synthetic spectrum.

For the $3280.7 \AA$ line, the red wing is severely affected by blends from the $\mathrm{Zr}$ II and Fe I transitions. By synthesising the region around the blue silver line using the derived metallicities of the stars, we found that the blending Fe line (3280.76 $\AA$ ) in most cases is overpredicted (red line in Fig. 4). Because our sample covers a large range of stellar parameters, we ran several syntheses, for a large number of stars spanning our entire parameter space with different $\log g f$ values for this line. In the end, we constrained the value of its transition probability so that it gives a reasonable fit to the entire sample. We thus altered the

\footnotetext{
${ }_{9}$ http://physics.nist.gov/PhysRefData/ASD/lines_form. html

${ }^{10}$ By adjusting the VALD "extract stellar" search the minimum $\log g f$ around the silver lines found is -3.4 dex, whereas using the VALD "extract all" yields a factor of two more lines reaching minimum $\log g f$ values of -9.7 .

${ }^{11}$ http://kurucz.harvard.edu/molecules.html
} 


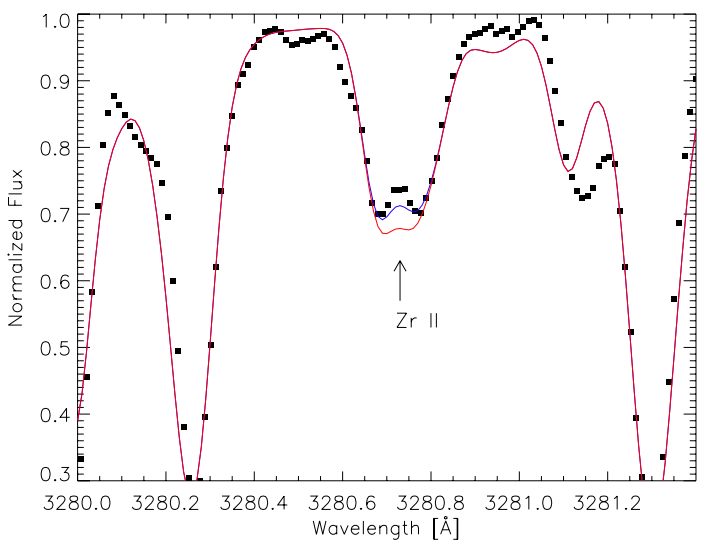

Fig. 5. Spectrum of HD 121004 with the results of two spectral syntheses with different $\log g f$ values for $\mathrm{Zr}$; in blue we plot the results for $-1.5 \mathrm{dex}$ and in red for $-1.1 \mathrm{dex}$. This demonstrates that a reduction in this zirconium line's $\log g f$ value was necessary to obtain a better synthesis and the correct silver abundances.

Fe I line $\log g f$ value from -2.231 dex to -2.528 dex. An example of this procedure is provided in Fig. 4 for the star HD 121004. The value listed in the VALD database $(-2.231)$ could be found in neither NIST nor the Fe line list of Fuhr et al. (1988).

Furthermore, we note that with this change we were also able to derive consistent solar abundances from both silver lines. Both solar spectra, the one observed with $\mathrm{UVES}^{12}$ and the Kurucz Solar Flux Atlas ${ }^{13}$ yielded silver abundances that differed by $\sim 0.3$ dex, with the bluer of the two lines giving the lowest silver abundance. The Kitt Peak solar spectrum ${ }^{14}$, which has the highest resolution $(R \sim 840000)$, also yields different abundances, of the order of 0.19 dex. The alteration of the Fe $\log g f$ to -2.528 dex led to an agreement between the two Ag lines/abundances within 0.04 dex of the two solar silver abundances and yielded a value of $0.93 \pm 0.02$ dex. This is in good agreement with the previous solar photospheric abundances summarised in Asplund et al. (2009, where $\log \epsilon(\mathrm{Ag})_{\odot}=$ 0.94 dex).

The synthesis of this region requires one more change to provide an acceptable fit. Based on equivalent width measurements of $\mathrm{Zr}$ II lines in the optical (see Sect. 6), we first determined the $\mathrm{Zr}$ abundance of each sample star, and used those values when synthesising the Ag line at $3280 \AA$. We noticed a similar feature as for the above-mentioned Fe line: the $\mathrm{Zr}$ abundance derived from the $\mathrm{Zr}$ II line in the red wing of the Ag line was always overestimated by $\sim 0.4$ dex (in all sample stars) when using the $\mathrm{Zr}$ abundance derived from the $\mathrm{Zr}$ optical lines. We then reduced the $\mathrm{Zr} \log g f$ of the $3280.735 \AA$ by 0.4 dex and obtained an overall much better fit (see the blue line in Fig. 5).

There are two additional important blends that contribute to the region around $3280.7 \AA$, namely that of Mn I and NH; however, for neither of these lines are changes needed to their atomic data, but they can be properly synthesised once we determined their abundances from other spectral lines/regions.

The $3382.9 \AA$ silver line has a strong Fe blend in its red wing (3382.985 $\AA$ ). This line is taken from the line list of Moore et al. (1966), because it was not found in either VALD or

\footnotetext{
${ }^{12} R \sim 85000$, http://www.eso.org/observing/dfo/quality/ UVES/pipeline/solar_spectrum.html

${ }^{13} R \sim 500000$, http: //kurucz.harvard. edu/sun.html

14 ftp://nsokp.nso.edu/pub/atlas/fluxatl/
}

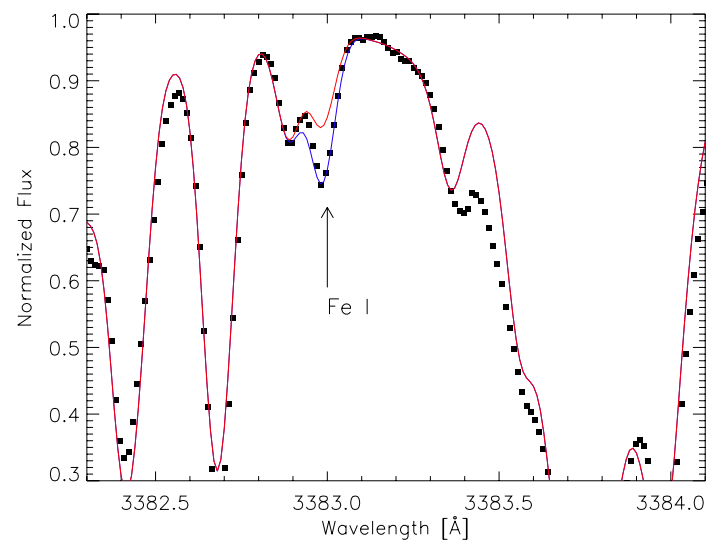

Fig. 6. Spectrum of HD 121004 (dots) to which two syntheses are fitted. The red indicates that the $\log g f$ value is too low, while the blue shows the properly adjusted $\log g f$ for the blending Fe I line.

NIST. However, Moore et al. (1966) only provide the excitation potential of this line, and we had to adjust the $\log g f$ empirically to obtain acceptable fits for this wavelength region. We adopted a $\log g f$ value of $-3.28 \pm 0.1 \mathrm{dex}$, which provides a good fit to the vast majority of our 71 sample stars.

The palladium line list was partially based on the line list published in Johnson \& Bolte (2002) and partly on VALD. The list required few (negligible) empirical adjustments and the solar value obtained from synthesising the line in the Kitt Peak solar spectrum was $\log \epsilon(\mathrm{Pd})_{\odot}=1.52 \mathrm{dex}$. As previously noted in Hansen \& Primas (2011), this value compares very well to the solar photospheric abundance of Pd summarised in Asplund et al. (2009), $\log \epsilon(\mathrm{Pd})_{\odot}=1.57 \mathrm{dex}$.

For $\mathrm{Ba}$ and $\mathrm{Eu}$, we used the hfs calculated relative oscillator strengths from McWilliam (1998) and Ivans et al. (2006), respectively. To derive accurate abundances, we applied a weighting to the lines from which we synthesised the abundances. For barium, we assigned the $5853 \AA$ line the highest weight (3) since this line is clean, and the $4554 \AA$ line has an intermediate weight (2) owing to the weak blends. Only when neither of the two aforementioned lines were detectable was the $4934 \AA$ line used (with weight 1 - otherwise it was given a weight 0 ) owing to the severe blends, yielding consistently lower abundances. Furthermore, we note that the $4554 \AA$ line tends to yield higher abundances $(\sim 0.1-0.15 \mathrm{dex})$ than the $5853 \AA$ line due to the presence of blends. Similarly, we assign weights to the Eu lines: $4129 \AA$ was given the highest weight (3) since it is clean, $4205 \AA$ an intermediate weight (2) owing to the weak blends, and the $6645 \AA$ line (weight 1 or 0 ) is only used when the two blue lines are neither detectable nor observed. The $4205 \AA$ Eu line yields abundances that on average 0.1 dex higher than those of the $4129 \AA$ line, while the abundances of the $6645 \AA$ line agree with the $4129 \AA$ derived ones. However, the $6645 \AA$ line is weak and generally only provides upper limits for our stars.

\section{Abundance analysis}

The abundances were calculated based on MARCS model atmospheres $^{15}$ (Gustafsson et al. 2008), which were interpolated to match the stellar parameters derived for our stars using the code written by Masseron (2006). Additionally, the 1D LTE synthetic

15 See http://www .marcs. astro.uu.se/ for model atmospheres in radiative and convective scheme (MARCS models). 
spectrum code MOOG (Sneden 1973, version 2009 including treatment of scattering) was applied to derive the stellar abundances. To date, neither NLTE corrections nor three-dimensional (3D) model effects have been studied for Ag or Pd. However, NLTE corrections can be found in the literature for $\mathrm{Sr}, \mathrm{Zr}, \mathrm{Ba}$, and Eu and we briefly comment on these when we discuss our results.

Owing to the severe line blanketing affecting the near$\mathrm{UV} /$ blue part of the spectra of all stars, blending plays a major role, thus spectrum synthesis is required to derive accurate abundances of $\mathrm{Ag}$ and Pd. Since hfs is substantial for the $\mathrm{Ba}$ and Eu abundances, we also derived their abundances via spectrum synthesis. For the other elements that we studied (Sr, Y, $\mathrm{Zr}$, and $\mathrm{Fe}$ ), we measured equivalent widths mostly in the redder parts of the spectra to avoid line blends. We measured most equivalent widths manually, by fitting Gaussian line profiles in IRAF (splot task), except for iron for which we used Fitline (François et al. 2003), due to the large number of Fe lines available in our spectra ${ }^{16}$.

\subsection{Correlation with stellar parameters?}

To ensure that our abundances are pure tracers of formation and evolution processes, and unaffected by spurious analytical effects and method biases, it is important to carefully investigate the trends of the derived abundances with temperature, gravity, and microturbulence.

Figure 7 shows that no trend with any of the three parameters is found, but it is evident that there is an abundance difference between dwarfs and giants. Non-local thermodynamic equilibrium effects could be one possible explanation of this difference; other possibilities could be mixing effects (Salaris et al. 2000; Korn 2008; Lind et al. 2008), microturbulent velocity, an incorrect treatment of the $T-\tau$ relation in the model atmospheres of giants, or unknown line blends in the spectra (Lai et al. 2008). This abundance difference cannot be explained by differences in the stellar evolutionary stages (cf. Preston et al. 2006).

The comparison of the $\mathrm{Pd}$ and $\mathrm{Ag}$ abundances to $[\mathrm{Fe} / \mathrm{H}]$ can be found in Hansen \& Primas (2011), where flat trends with metallicity were found. This means that the abundances are not biased by the stellar parameters or the methods applied to determine these, and our abundances can be seen as pure tracers of the formation processes. This allows us to apply the abundances as direct indicators of the chemical evolution of the Galaxy.

\subsection{Error estimation}

The final error in the derived abundances stems from uncertainties in the stellar parameters, the synthesis/equivalent width measurements, and the continuum placement. The stellar parameter uncertainties are $\left(T_{\text {eff }} / \log g /[\mathrm{Fe} / \mathrm{H}] / \xi\right): \pm 100 \mathrm{~K} / 0.2-$ $0.25 \mathrm{dex} / 0.1-0.15 \mathrm{dex} / 0.15 \mathrm{~km} \mathrm{~s}^{-1}$ (cf. Sect. 3.1). Their effect on the abundances was constrained by running different models in which each parameter was varied by its corresponding uncertainty, one at a time.

Furthermore, since we synthesised both Pd and Ag transitions, we needed to include the uncertainty in the continuum placement (about $\pm 0.05 \mathrm{dex}$ ) and the possible incompleteness of

\section{The abundances are calculated as:}

$[\mathrm{A} / \mathrm{B}]=\log (\mathrm{A} / \mathrm{B})-\log (\mathrm{A} / \mathrm{B})_{\odot}$, where $\log \epsilon(A)=\log \left(\frac{N_{\mathrm{A}}}{N_{\mathrm{H}}}\right)+12$,

where $N_{\mathrm{A}}$ and $N_{\mathrm{H}}$ are the number densities of absorbing atoms of element $\mathrm{A}$ and hydrogen, respectively. We adopted a scale where the number of $\mathrm{H}$ atoms is set to $10^{12}$.
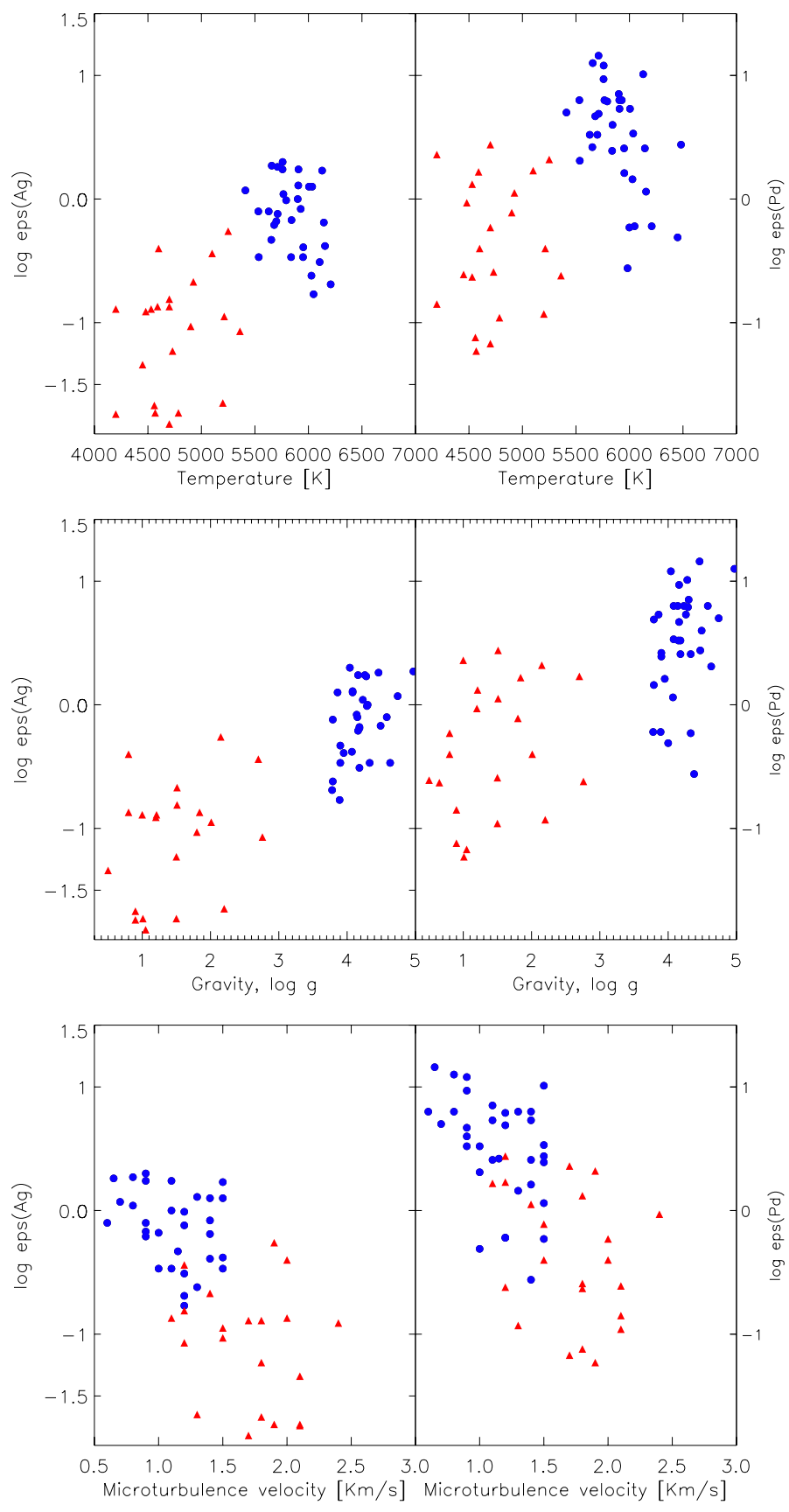

Fig. 7. Abundances of $\mathrm{Ag}$ (left) and Pd (right) compared to stellar parameters. They show a clear division between the dwarfs and the giants. No trends could be fitted owing to the very large $\chi^{2}$.

stellar model atmospheres, the synthetic code, and the line list (i.e. missing atomic data), which all together sums up to an uncertainty of \pm 0.1 dex. Adding all three contributions in quadrature yields uncertainties of the order of \pm 0.2 dex and \pm 0.25 dex in the $\mathrm{Pd}$ and $\mathrm{Ag}$ abundances, respectively. The average error in the equivalent width measurements of $\mathrm{Sr}$ and $\mathrm{Y}$ is around $2.5 \mathrm{~m} \AA$ and slightly larger for $\mathrm{Zr}(\sim 4 \mathrm{~m} \AA)$. These errors were incorporated into the total uncertainty in the abundances shown in the figures in Sect. 6.

Propagating the uncertainties in the heavy element abundances derived from equivalent width measurements and stellar parameters resulted in abundance errors of $0.1-0.3$ dex. Details can be found in Tables C. 1 and C.2. 
Table 2. Elements and the process that they trace at solar metallicity.

\begin{tabular}{lccc}
\hline \hline Elements & Formation process [1] & Reference & r-process fraction [8] \\
\hline $\mathrm{Sr}$ & $85 \%$ s-process (weak s-process) & {$[1,3,6]$} & $2.7 \%$ \\
$\mathrm{Y}$ & $92 \%$ s-process (in part weak s-process) & {$[1,3,6]$} & $5 \%$ \\
$\mathrm{Zr}$ & $83 \%$ s-process (low weak s-process) & {$[1,6]$} & $9.2 \%$ \\
$\mathrm{Pd}$ & $54 \%$ r-process (some (< 67\%) weak r-process) & {$[1,2]$} & $46.9 \%$ \\
$\mathrm{Ag}$ & $80 \%$ r-process (mainly (> 71\%) weak r-process) & {$[1,2,5]$} & $77.9 \%$ \\
$\mathrm{Ba}$ & $81 \%$ s-process, (main s-process) & {$[1,4,7]$} & $11.3 \%$ \\
$\mathrm{Eu}$ & $94.2 \%$ r-process (main r-process) & {$[1]$} & $94 \%$ \\
\hline
\end{tabular}

References. [1] Arlandini et al. (1999), [2] Farouqi et al. (2009), [3] Heil et al. (2009), [4] Lodders et al. (2009), [5] Montes et al. (2007), [6] Pignatari et al. (2010), [7] Sneden et al. (2008), and [8] Bisterzo et al. (2011) for a comparison to more recent r-process fractions.

\section{Indications of a second r-process}

To characterise the formation process of Pd and Ag, we compare their abundances to those of various different elements that trace the weak/main s-process and the main r-process. This comparison allows us to detect either similarities or differences between the yet unidentified formation process of Pd and $\mathrm{Ag}$ and the known formation processes of the elements we compare to. For this purpose, we selected the following tracer elements, which at solar metallicity are created by the process we have listed in Table 2. This means that a correlation of Ag with $\mathrm{Ba}$ around solar metallicity would indicate that $\mathrm{Ag}$ had a common formation process to $\mathrm{Ba}$, which in this case would be the main s-process. However, at low metallicity this picture changes: Sr, Y (and Zr) could be created by charged particle freeze-outs (Kratz et al. 2008b; Farouqi et al. 2009), and Ba mainly by the main $\mathrm{r}$-process. We find indications that $\mathrm{Zr}$ also receives weak r-process contributions at low $([\mathrm{Fe} / \mathrm{H}]<-2.5)$ metallicities, which agrees with Farouqi et al. (2009; see also Sect. 7).

\subsection{Chemical evolution trends of $\mathrm{Sr}-\mathrm{Eu}$}

We first compare the elemental abundances of $\mathrm{Sr}-\mathrm{Eu}$ with $\mathrm{Fe}^{17}$ to follow the chemical evolution of these elements, and detect the onset of the various formation processes. We also compare our derived abundances to other studies from the literature, which include measurements for some or all of the elements studied here. The following five large abundance studies were chosen: Johnson \& Bolte (2002, J02), Barklem et al. (2005, B05), François et al. (2007, F07), Bonifacio et al. (2009, B09), and Roederer $\left(2009, \mathrm{R} 09^{*}\right)$. The last (R09*) is a compilation of previous studies by Edvardsson et al. (1993), Fulbright (2000), Nissen \& Schuster (1997), and Stephens \& Boesgaard (2002). As mentioned in Sect. 2, we include and compare with some r-process enhanced stars. These are: $\mathrm{BD}+17^{\circ} 3248$ (Cowan et al. 2002), CS 22892-052 (Sneden et al. 2003), and CS 31082-001 (Hill et al. 2002, included in our sample). These are clearly labelled in the figures.

Starting with the lightest element, Sr, we see that down to $[\mathrm{Fe} / \mathrm{H}]=-2.5,[\mathrm{Sr} / \mathrm{Fe}]$ presents a relatively clean and flat trend with a mean value around 0.14 dex (see Fig. 8). Below this metallicity, the scatter becomes dominant. Only three stars deviate from this picture (HD 175179, HD 195633, and G005-040), for which only upper limits were attainable from near-UV lines (no spectra covering the wavelength range 3800-4800 ̊ were available in the ESO archive).

\footnotetext{
$\overline{17 \text { All abundances are available in Tables C.1, C.2 }}$
}

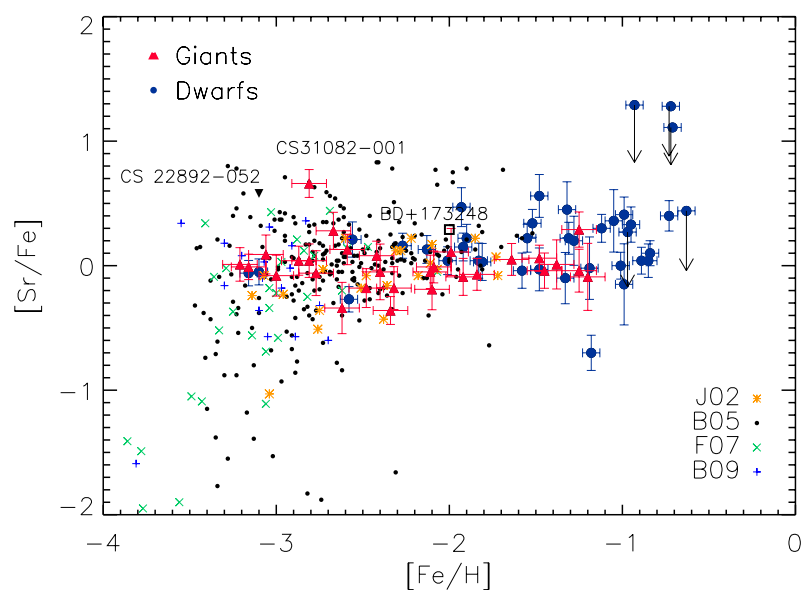

Fig. 8. $[\mathrm{Sr} / \mathrm{Fe}]$ as a function of $[\mathrm{Fe} / \mathrm{H}]$ for the entire sample, compared to Johnson \& Bolte (2002, J02 - orange asterisk), Barklem et al. (2005, B05 - black dots), the "First Stars" giants François et al. (2007, F07 - green $\times$ ), and dwarfs Bonifacio et al. (2009, B09 - purple +), respectively. The dwarfs from our sample are shown as filled blue circles, while filled red triangles represent our giants. Three very enhanced stars are shown and labelled in this and the following figures: BD $+17^{\circ} 3248$ (Cowan et al. 2002, open black square), CS 22892-052 (Sneden et al. 2003, filled black triangle), and CS 31082-001 (Hill et al. 2002, also analysed in this study, hence the red triangle). Arrows indicate upper limits to the abundances. A flat trend of $[\mathrm{Sr} / \mathrm{Fe}]$ is seen down to $[\mathrm{Fe} / \mathrm{H}] \sim-2.5$, below which the scatter becomes dominant.

The trend for yttrium is also seen to be flat down to $[\mathrm{Fe} / \mathrm{H}]=$ -2.5 dex (Fig. 9). We find the same increase in star-to-star scatter of $[\mathrm{Y} / \mathrm{Fe}]$ with decreasing $[\mathrm{Fe} / \mathrm{H}]$ as detected in Roederer et al. (2010). However, the average Y abundance is sub-solar. In general the abundance predictions of the $\mathrm{Sr} / \mathrm{Y}$-ratio from $\mathrm{SN}$ models are found to be very high, most likely due to incorrect solar scaled residuals ${ }^{18}$. A too-low solar abundance of $\mathrm{Y}$ could have explained this, but this does not seem to be the case, since the solar photospheric and meteoritic Y abundance agree to within 0.04 dex (Asplund et al. 2009), making this a trustworthy value.

The zirconium abundance distribution is also flat and found to have a mean value of 0.2 dex down to a metallicity of at least -2.5 dex (see Fig. 10). The scatter in $[\mathrm{Zr} / \mathrm{Fe}]$ below $[\mathrm{Fe} / \mathrm{H}]=$ -2.5 is less pronounced than for $[\mathrm{Sr} / \mathrm{Fe}]$, which may be due to there being fewer $\mathrm{Zr}$ abundance determinations at low metallicities compared to, e.g., Sr. One can see from Table A.3, that the

18 The Sr/Y-ratio can be correctly predicted by the high-entropy wind models (Farouqi et al. 2009), where these residual assumptions are not considered. 


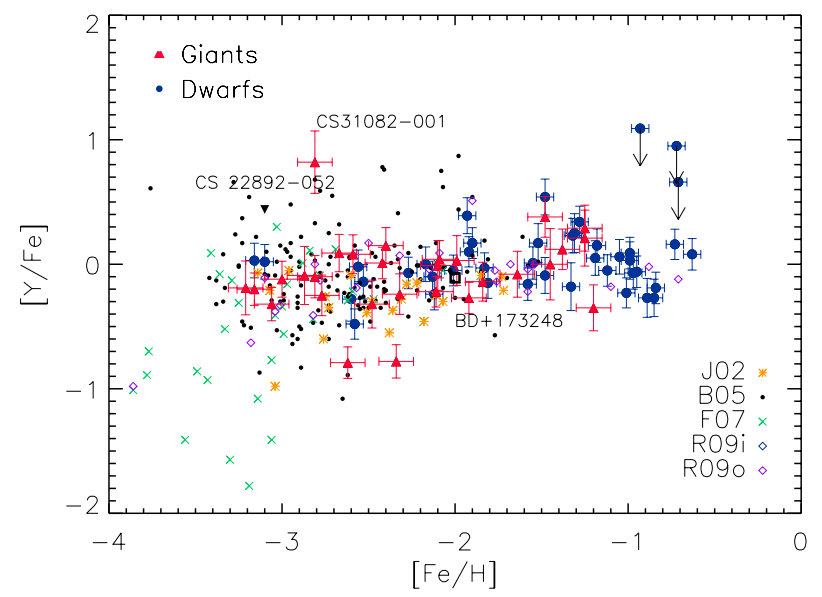

Fig. 9. $[\mathrm{Y} / \mathrm{Fe}]$ vs. $[\mathrm{Fe} / \mathrm{H}]$ similar comparison samples as in Fig. 8, but also including a fourth sample - Roederer $(2009$, R09i/o) - shown as blue/purple open diamonds indicating stars belonging to the inner/outer halo, respectively. The enhanced stars agree with the other comparison samples as well as our sample. However, CS 31082-001 is seen to be particularly enhanced in $\mathrm{Y}$. $[\mathrm{Y} / \mathrm{Fe}]$ shows almost no variation with metallicity down to $[\mathrm{Fe} / \mathrm{H}] \sim-2.5$ dex.

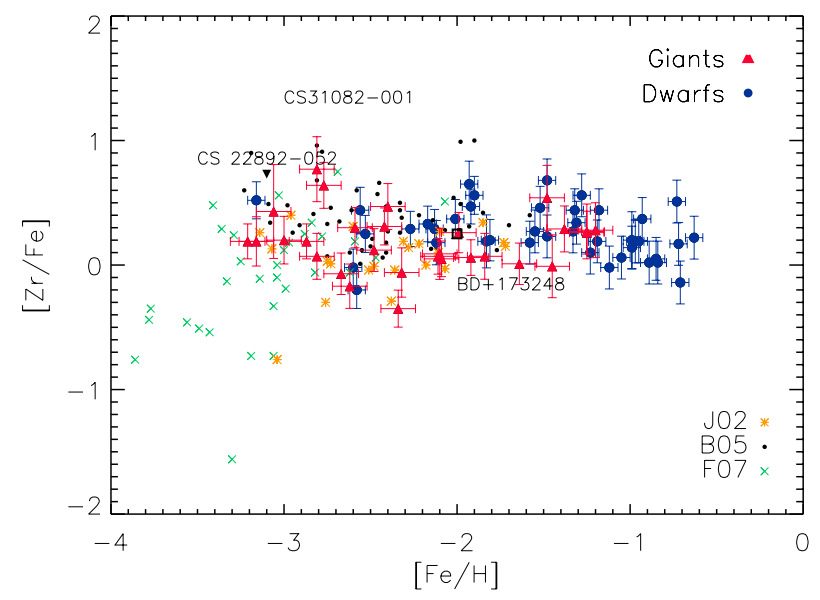

Fig. 10. $[\mathrm{Zr} / \mathrm{Fe}]$ as a function of $[\mathrm{Fe} / \mathrm{H}]$. $\mathrm{Zr}$ does not vary much with metallicity. Symbols and colour are the same as in Fig. 8.

$\mathrm{Zr}$ lines are intrinsically much weaker than, e.g., the $\mathrm{Sr}$ and $\mathrm{Ba}$ resonance lines.

Figure 11 shows the evolutionary trend of $[\mathrm{Ba} / \mathrm{Fe}]$ vs. $[\mathrm{Fe} / \mathrm{H}]$ which is characterised by a large scatter ( $>2$ dex) below a metallicity of $[\mathrm{Fe} / \mathrm{H}]=-2.0$. The large scatter can be interpreted as an indication of different yields from one enrichment event to another, creating an inhomogeneous interstellar medium (ISM). However, it could also point towards several formation processes being at work and releasing very different elemental ratios into the ISM. Even when correcting the derived $\mathrm{Ba}$ abundances for NLTE effects (see Andrievsky et al. 2009), the scatter is far in excess of any possible uncertainty stemming from observations and model assumptions. It is therefore a possible indication that different formation processes are at play. Figure 12 shows a large spread in the europium abundances.

The evolutionary trends of both $[\mathrm{Pd} / \mathrm{Fe}]$ and $[\mathrm{Ag} / \mathrm{Fe}]$ relative to $[\mathrm{Fe} / \mathrm{H}]$ were previously presented in Hansen \& Primas (2011) and were found to be flat and scattered, similarly to the other five elements discussed above. Here, we thus decided to show new plots of Pd and Ag abundances, relative to their neighbouring elements (see following sub-sections).

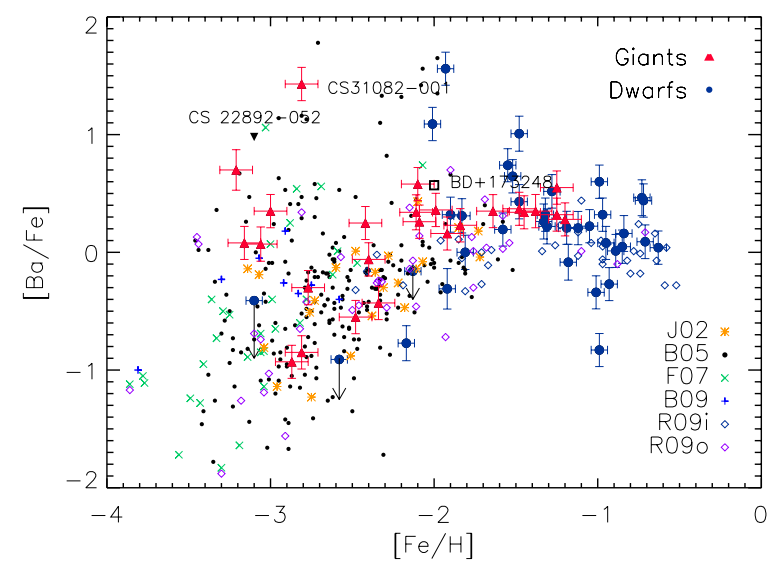

Fig. 11. $[\mathrm{Ba} / \mathrm{Fe}]$ plotted vs. $[\mathrm{Fe} / \mathrm{H}]$. Below $[\mathrm{Fe} / \mathrm{H}] \sim-2.0$, a very large scatter in all samples is seen. The very large scatter is indicative of a poorly mixed ISM. Symbols and colour coding as in Fig. 8.

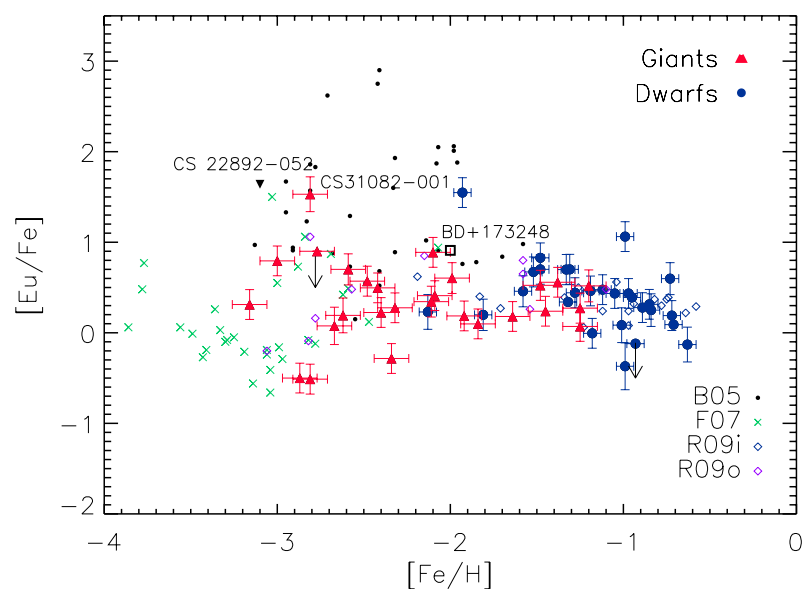

Fig. 12. $[\mathrm{Eu} / \mathrm{Fe}]$ as a function of metallicity. A very large scatter is seen at all metallicities (also within the various samples). Symbols and colour coding as in Fig. 8.

We note that, in general, the r-process enhanced stars follow the overall trends, but fall on the upper abundance envelope as one would expect from their enhancements. For CS 31082001 , we re-derived all abundances and found them to agree very well with the results of Hill et al. (2002). The only exception is yttrium, which we propose is caused by uncertainties in the continuum placement $( \pm 0.1 \mathrm{dex})$ and the profile fitted. The $\mathrm{Y}$ lines to which we fit Gaussian profiles are very sensitive to the exact shape and broadening of the profile, and we can only reproduce the observed spectral line by fitting much broader line profiles to the Y lines than the surrounding spectral lines. The offset in line profile between the $\mathrm{Y}$ lines and the nearby other spectral lines introduces an 0.3 dex abundance offset in our Y abundance. We can attribute our higher $\mathrm{Y}$ abundance compared to that derived in Hill et al. (2002) to a combination of uncertainties and offsets.

The star-to-star abundance scatter revealed by all the elemental trends discussed here points to a rather inhomogeneous ISM below a metallicity of -2.5 (see Sect. 6.4 for further discussion). Below this metallicity, the varying abundances indicate that the stars have been affected by different productions (or processes) from various nucleosynthetic events. The main contribution at these low metallicities must come from primary processes, since the sites of the secondary processes (the s-processes) have not yet had enough time to both reach the evolutionary stages where they yield s-process contributions and 

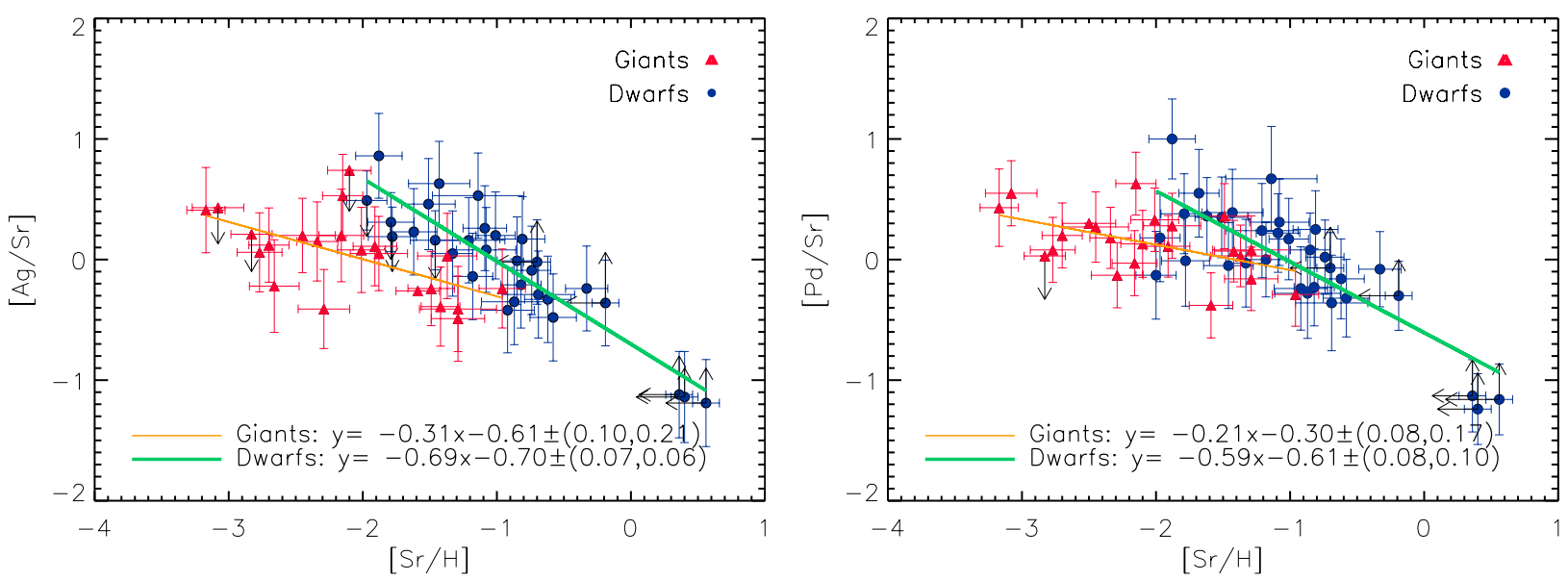

Fig. 13. $[\mathrm{Ag} / \mathrm{Sr}]$ (left $)$ and $[\mathrm{Pd} / \mathrm{Sr}]($ right $)$ as a function of $[\mathrm{Sr} / \mathrm{H}]$ is shown here for both dwarfs (filled blue circles) and giants (filled red triangles). An anti-correlation is seen in this figure, which is strongest for the dwarfs (see the slopes in the figure). The values given in parenthesis are the uncertainties in the linear fits: the first number is the error in the slope, the second number is the uncertainty in the intersection with the $y$-axis.

have their yields incorporated into later generations of stars. This is why any monitoring of the r-process is carried out most efficiently below $[\mathrm{Fe} / \mathrm{H}]=-2.5$. From Figs. $8-11$, the s-process might start around $[\mathrm{Fe} / \mathrm{H}]=-2.5$ dex, since we see a change in the abundance behaviour (trend flattening/lower scatter) at this metallicity. Unfortunately, our data do not allow us to identify the metallicity for the onset of the weak s-process, a problem that we discuss further in Sect. 6.3.

\subsection{Correlations and anti-correlations}

We now turn to a different set of abundance plots, of the type $[\mathrm{A} / \mathrm{B}]$ vs. $[\mathrm{B} / \mathrm{H}]$ (where $\mathrm{A}$ and $\mathrm{B}$ are two of the neutron-capture elements under investigation), to see whether and how they (anti-) correlate with each other. This is determined by the abundance trends to which we fit lines. The slopes determine the anti-/correlation. The fitting of linear trends has been made to all points (stars) taking their uncertainties into consideration, and the uncertainties in the fits are expressed in the figures in parentheses. These plots are powerful diagnostics for constraining formation processes and can help us to identify similarities and differences among the neutron-capture elements. If $\mathrm{A}$ and $\mathrm{B}$ correlate (i.e. the $[\mathrm{A} / \mathrm{B}]$ ratio is flat across the spanned values of $[\mathrm{B} / \mathrm{H}]$ ), it means that they grow in the same way (constant ratio) and that they are most likely created by the same process. If they anti-correlate (e.g. [A/B] decreases with increasing $[\mathrm{B} / \mathrm{H}])$, this is usually interpreted in terms of their having different amounts of $\mathrm{A}$ and $\mathrm{B}$, hence different processes being responsible for their formation. To define our terminology, the strengths of the correlations can be described as follows; a weak/mild anticorrelation is stated for slopes between -0.25 and -0.5 and a strong anti-correlation is assigned to negative slopes around or steeper than -0.5 . We choose hydrogen $(\mathrm{H})$ as our reference element because we wish to focus only on the formation processes of elements A and B. Had we selected iron instead, the interpretation of the plots would have become more complex because of the different sites contributing to the formation of iron.

In the following, there are two important factors to bear in mind, namely the difference between dwarfs and giants and that below $[\mathrm{Fe} / \mathrm{H}]<-2$ dex the silver lines could only be detected in giant stars. The giants might have been affected by NLTE or mixing effects, whereas the inclusion of the dwarfs may affect our constraints on the formation processes. The giants could be affected by almost pure r-process yields, whereas the dwarfs might carry a mixture of $r$ - and s-process yields. Therefore, we need to test the purity of the r-process as we do in Sect. 7. Furthermore, it is very important to look for differences in the behaviour of the $\mathrm{Ag}$ and $\mathrm{Pd}$ abundance ratios in dwarf and giant stars (see Sect. 6.4).

Now focusing on the formation process of $\mathrm{Pd}$ and $\mathrm{Ag}$, we start by comparing these two elements to Sr, Y, and Zr, which may be formed by the weak s-process elements or charged particle freeze-out (depending on metallicity).

In general, Figs. 13-15 have one common feature, i.e. they all clearly show that the elements plotted in each graph anticorrelate. Although these anti-correlations are characterised by slightly different (negative) slopes, all of these plots agree that neither $\mathrm{Pd}$ nor $\mathrm{Ag}$ are formed by the same mechanism that produced $\mathrm{Sr}$, Y, or $\mathrm{Zr}$ (i.e. weak s-process or charged particle freeze-outs). However, these negative slopes do not merely differ randomly between the elements, but there seems to be a clear decreasing trend (i.e. the slopes become shallower) going from $\mathrm{Sr}$ to $\mathrm{Y}$ and then to $\mathrm{Zr}$. The slopes derived by fitting the data-points in $[\mathrm{Ag}, \mathrm{Pd} / \mathrm{Zr}]$ are between -0.37 and $-0.18 \pm 0.07$, which thus indicate that there is only a mild anti-correlation. We interpret this as an indication that $\mathrm{Zr}$ may be produced (at least in part) by the same formation process producing $\mathrm{Pd}$ and $\mathrm{Ag}$.

When comparing Ag to Pd (see Fig. 16), it becomes difficult to draw a firm conclusion about the exact trend of their abundance ratio $[\mathrm{Ag} / \mathrm{Pd}]$ as a function of $[\mathrm{Pd} / \mathrm{H}]$. Despite the slopes overplotted on the graph being indicative of a very mild anticorrelation, they may be misleading especially since they take into account giants and dwarfs separately. If one were to ignore these slopes and consider the entire sample as a whole, we could argue that we find a flat $[\mathrm{Ag} / \mathrm{Pd}]$ trend, especially when considering the associated error-bars and excluding upper limits. The latter is also supported by our earlier finding of an almost 1:1 linear slope between $[\mathrm{Ag} / \mathrm{H}]$ vs. $[\mathrm{Pd} / \mathrm{H}]$ (Hansen \& Primas 2011), which strongly indicates a common origin for these two elements.

If we now consider how $\mathrm{Ag}$ and $\mathrm{Pd}$ compare to $\mathrm{Ba}$ (Fig. 17), which is the most representative tracer of the main s-process, we see that both $\mathrm{Ag}$ and Pd strongly anti-correlate with Ba, which excludes the main s-process as one of the possible production channels responsible for the formation of $\mathrm{Ag}$ and $\mathrm{Pd}$. At low metallicity $([\mathrm{Fe} / \mathrm{H}]<-2.5 \mathrm{dex}), \mathrm{Ba}$ is created by the main r-process, which indicates that $\mathrm{Pd}$ and $\mathrm{Ag}$ are also not created by 

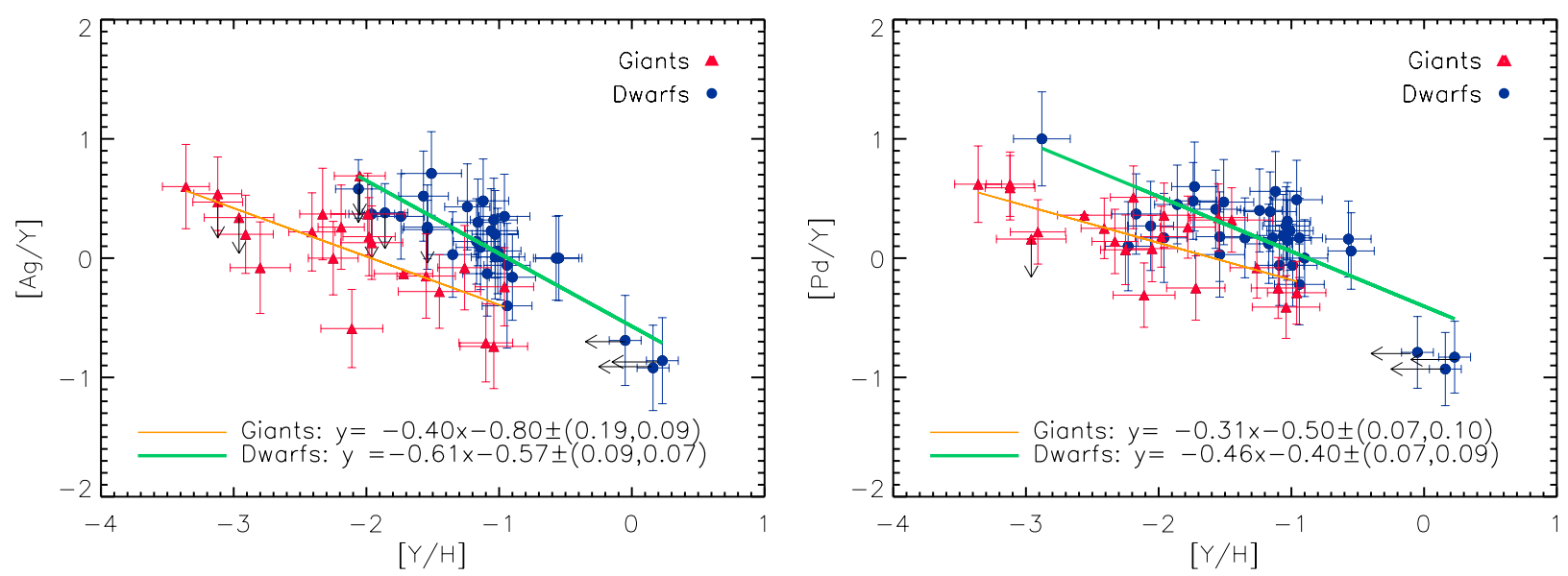

Fig. 14. Left: $[\mathrm{Ag} / \mathrm{Y}]$ as a function of $[\mathrm{Y} / \mathrm{H}]$. Right: $[\mathrm{Pd} / \mathrm{Y}]$ vs. $[\mathrm{Y} / \mathrm{H}]$. Legend is described in Fig. 13 and shown in the figure. Anti-correlations between the weak s-process element $\mathrm{Y}$ and $\mathrm{Ag}$ and $\mathrm{Pd}$ are seen in this figure.
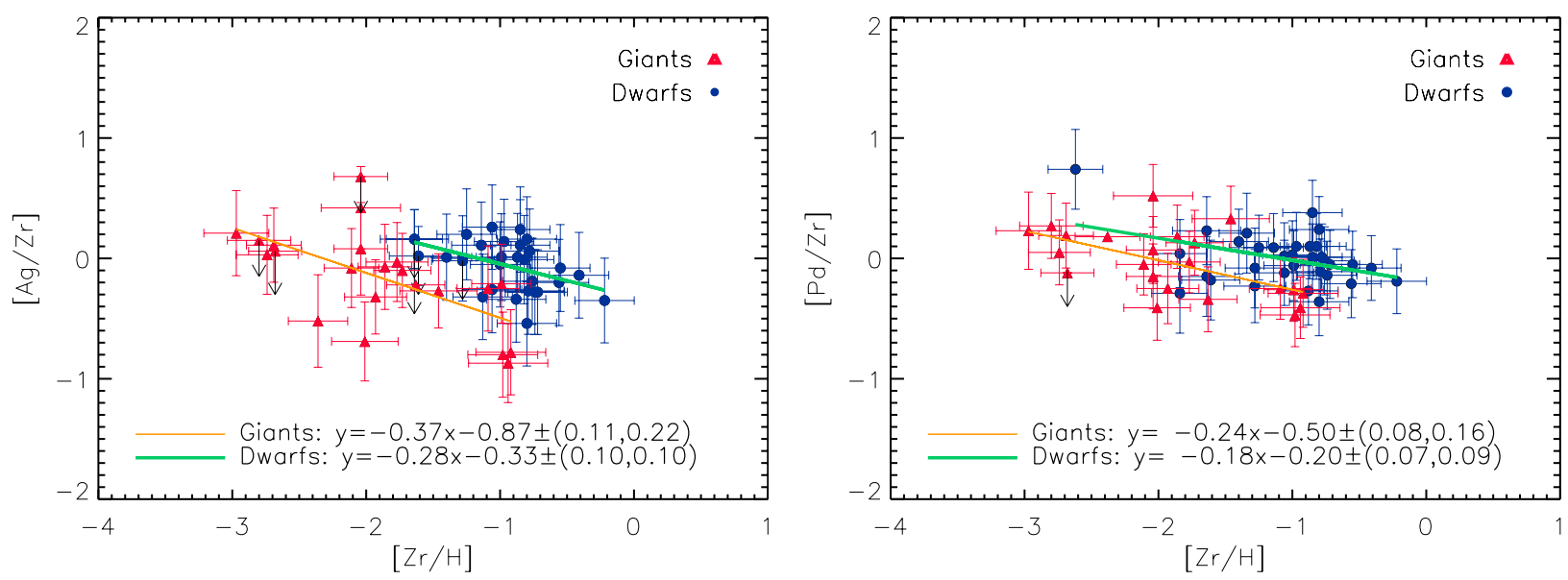

Fig. 15. $[\mathrm{Ag} / \mathrm{Zr}]$ and $[\mathrm{Pd} / \mathrm{Zr}]$ vs. $[\mathrm{Zr} / \mathrm{H}]$ to the left and right, respectively. The trend of the fitted line is only slightly negative, which could be interpreted as a slight correlation, but the abundances clump. Upper limits to the abundances are indicated by arrows. The formulas of the lines fitted are given in the lower left corner for giants and dwarfs, respectively.

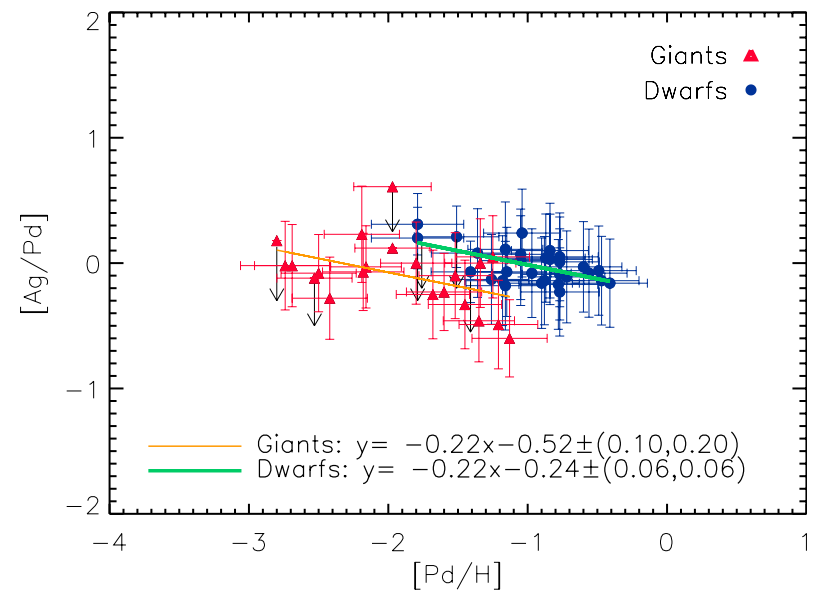

Fig. 16. An almost flat trend (correlation) is seen in the figure showing $[\mathrm{Ag} / \mathrm{Pd}]$ as a function of $[\mathrm{Pd} / \mathrm{H}]$, which is indicative of a similar origin of $\mathrm{Ag}$ and $\mathrm{Pd}$.

the main r-process, although we compare them to Eu to confirm this finding. Finally, Fig. 18 shows that strong anti-correlations of $\mathrm{Ag}$ and $\mathrm{Pd}$ are found with $\mathrm{Eu}$, which means that the process forming Pd and Ag cannot be the main r-process. We cannot, however, exclude that $\mathrm{Ag}$ and $\mathrm{Pd}$ are partly produced by the main r-process.

Therefore, the formation process of $\mathrm{Pd}$ and $\mathrm{Ag}$ is neither a charged particle freeze-out, a weak, main s-process, nor a main r-process. Both $\mathrm{Ag}$ and $\mathrm{Pd}$ are seen to form at extremely low metallicity $([\mathrm{Fe} / \mathrm{H}]<-3)$. These results, combined with the predictions of Montes et al. (2007), Kratz et al. (2008a), and Farouqi et al. (2009), indicate that their formation process must be of primary and likely r-process nature, but we need to resort to model comparisons in order to characterise this second r-process.

As mentioned at the beginning of this sub-section, one needs to keep in mind two caveats when discussing these abundances: i) we derived all abundances based on 1D LTE model atmospheres and spectral syntheses; ii) we were able to track the evolution of Ag down to the lowest metallicities only with giant stars. We adopted the former approach because NLTE corrections are available for only some of the elements investigated here, namely Sr (e.g. Belyakova \& Mashonkina 1997; Andrievsky et al. 2011; Bergemann et al. 2012), Zr, Ba (e.g. Andrievsky et al. 2009), and to some extent Eu. However, no NLTE corrections have been calculated for our two key elements $\mathrm{Pd}$ and $\mathrm{Ag}$, and only a few for $\mathrm{Y}$ and $\mathrm{Zr}$ (Velichko et al. 2010). Because we use primarily [A/B] ratios (where A can be either $\mathrm{Ag}$ or $\mathrm{Pd}$, and $\mathrm{B}$ is one of the other neutron-capture elements), we decided to keep a 1D LTE consistency across all 

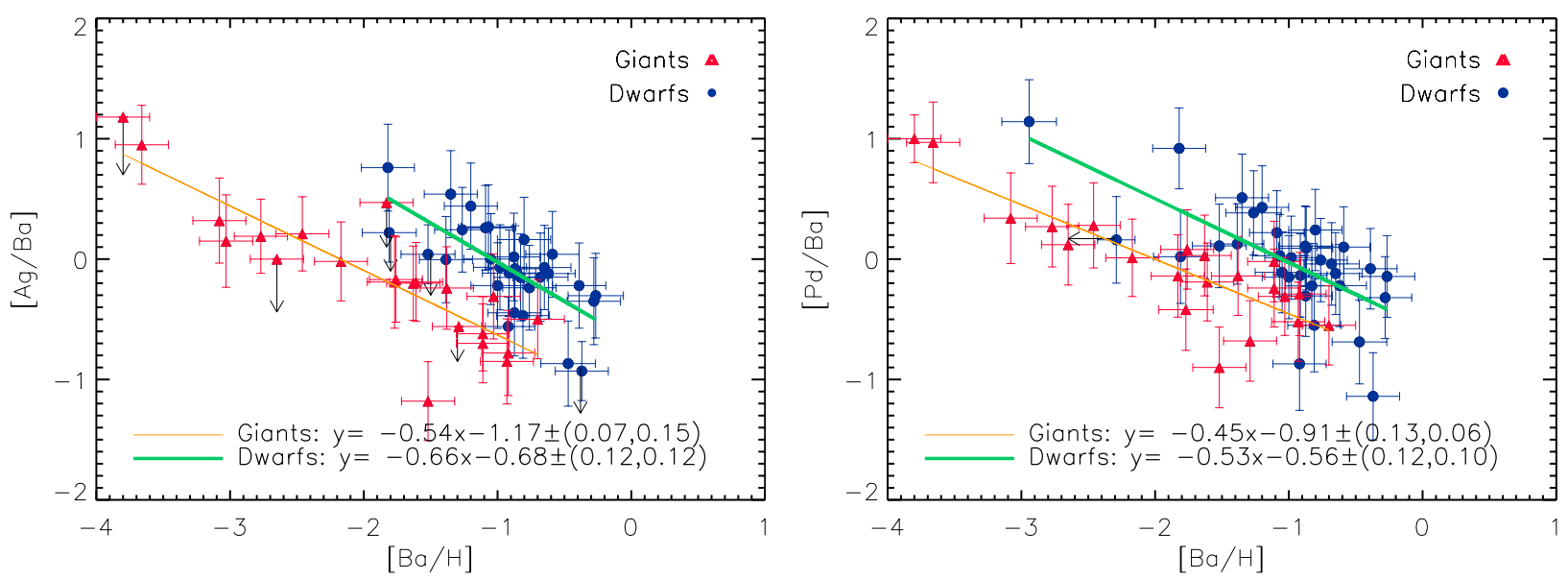

Fig. 17. A strong anti-correlation is seen in this plot of $[\mathrm{Ag} / \mathrm{Ba}]$ vs. $[\mathrm{Ba} / \mathrm{H}]$ and $[\mathrm{Pd} / \mathrm{Ba}]$ vs. $[\mathrm{Ba} / \mathrm{H}]$. Silver and palladium are therefore not main s-process elements.
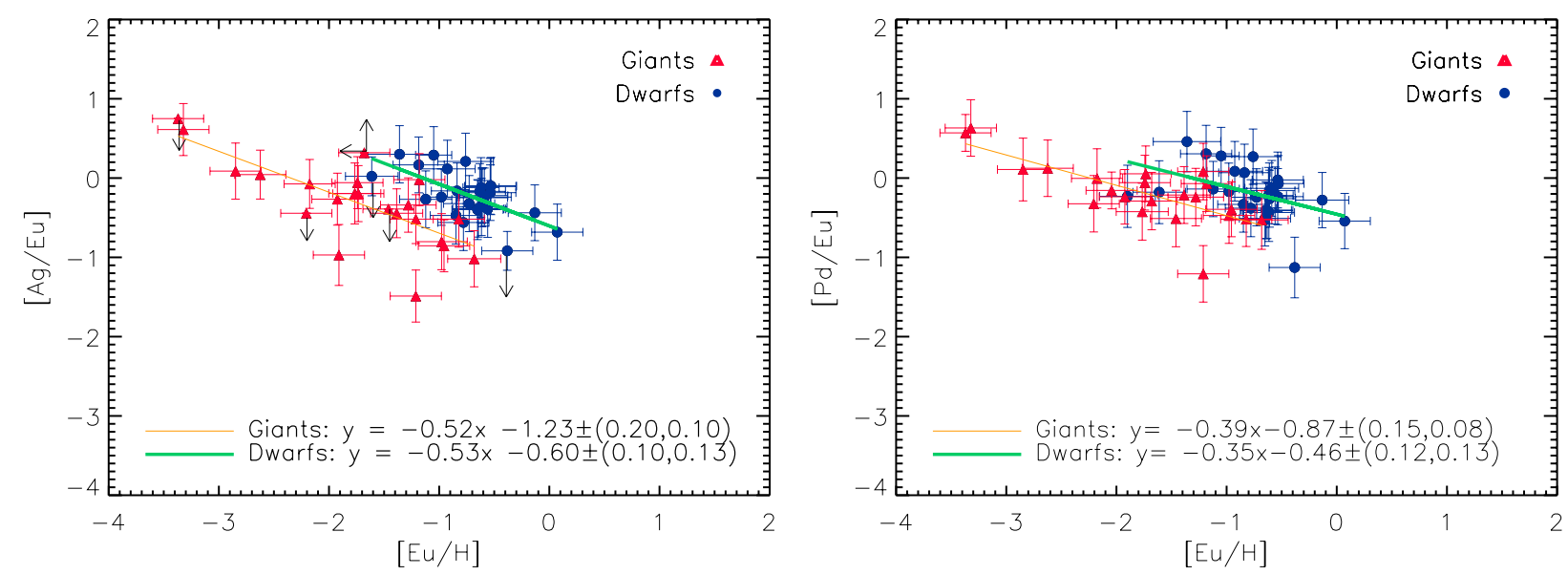

Fig. 18. To the left: $[\mathrm{Ag} / \mathrm{Eu}]$ plotted as a function of $[\mathrm{Eu} / \mathrm{H}]$, showing a clear and strong anti-correlation. To the right: $[\mathrm{Pd} / \mathrm{Eu}] \mathrm{vs}$. [Eu/H]. This means that $\mathrm{Ag}$ and Eu are not synthesised by the same process, nor are Pd and Eu. Silver and palladium are therefore not produced by the main r-process.

ratios, instead of correcting only some elements. We are, however, fully aware of the importance of NLTE corrections, and that would ideally be a better way to proceed, were NLTE corrections to become available for all elements. As for the latter, dwarfs and giants show in general very similar trends (see Figs. 13-18), with the dwarfs having higher abundance values than the giants at similar metallicities. However, the overall good agreement between dwarfs and giants suggests that the process creating $\mathrm{Ag}$ and Pd is likely to be the same at all metallicites.

\subsection{Formation processes and transitions around $\mathrm{Zr}$}

Zirconium and strontium clearly share a common formation process at low metallicities down to and even slightly below $[\mathrm{Zr} / \mathrm{H}]=-3$ (see the flat correlation for giants in Fig. 19). A similar trend is found when comparing yttrium to zirconium and yttrium to strontium. However, at higher $[\mathrm{Fe} / \mathrm{H}]$ and $[\mathrm{Sr} / \mathrm{H}]$ abundances above -1 dex, we find an anti-correlation between $\mathrm{Sr}$ and $\mathrm{Zr}$ for the dwarfs. At higher metallicities, this can indicate differences in the formation process - or a difference between the process primarily responsible for the formation of the two elements.

Zirconium and barium seem to have different origins, as shown in Fig. 20 (Zr; e.g. charged particle freeze-out or weak r-process vs. Ba; main r-process origin at low metallicities).
These findings confirms those of Farouqi et al. (2009) and Kratz et al. (2008a, see their Fig. 4), who found a low-entropy chargedparticle freeze-out process to be the primary formation process of $\mathrm{Sr}, \mathrm{Y}$, and $\mathrm{Zr}$ at low metallicity. Here, we find indications of $\mathrm{Zr}$ being created in a slightly different way from $\mathrm{Sr}$ and Y. Similar trends are also seen for $[\mathrm{Sr} / \mathrm{Ba}]$ and $[\mathrm{Y} / \mathrm{Ba}]$ ratios, where the giants show clear anti-correlations. The trends for giants were already reported by e.g. François et al. (2007). For the dwarfs, this trend is less pronounced and they have a greater scatter in the abundances. From the dwarfs' trends, we might conclude that around $[\mathrm{Ba} / \mathrm{H}]=-2$ the s-process yields from asymptotic giant branch stars are no longer negligible formation sites of $\mathrm{Ba}$, and that the larger scatter is evidence of multiple formation sources. Comparing the giant abundances of $\mathrm{Zr}$ to Eu shows that like $\mathrm{Pd}$ and $\mathrm{Ag}, \mathrm{Zr}$ is not produced by the main r-process at higher metallicites (see Fig. 20), although we note that $\mathrm{Zr}$ and Pd follow a weaker anti-correlation with Eu than Ag does.

In the solar system, $\mathrm{Zr}$ appears to have been partly produced by the weak and main s-processes (as well as there being a minor contribution from the weak/second r-process), owing to the correlations (and only mild anti-correlation) of $\mathrm{Zr}$ with $\mathrm{Sr}, \mathrm{Pd}, \mathrm{Ag}$, and $\mathrm{Ba}$. At low metallicities, the s-process contribution to $\mathrm{Sr}$, $\mathrm{Y}$ (and $\mathrm{Zr}$ ) is substituted with a charged particle freeze-out creation. These statements are confirmed in Sect. 7. This means that 


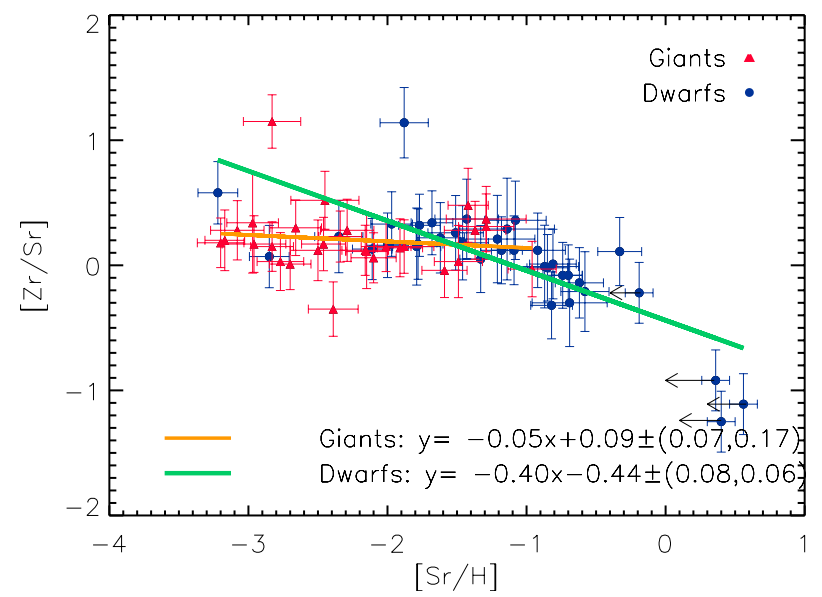

Fig. 19. $\mathrm{Zr}$ and $\mathrm{Sr}$ correlate in the metal-poor giants which indicates a similar formation process of these two elements. This is in agreement with the findings of Farouqi et al. (2009) and Kratz et al. (2008b). At higher metallicities $([\mathrm{Sr} / \mathrm{H}]>-1)$ the formations of $\mathrm{Sr}$ and $\mathrm{Zr}$ differ. The upper limits are due to the before mentioned lacking visual spectra of the three stars (see text).

$\mathrm{Zr}$ may represent a transition in the periodic table around atomic number 40 from the weak s-process/charged particle freeze-out process (depending on metallicity) to the weak r-process. This second r-process could be responsible for the creation of elements in the atomic number range 40-50. However, this process would cease to create elements somewhat below barium. This upper limit is uncertain owing to the lack of elements investigated (observationally in large samples) in the range 48-55. We note that a natural end to the weak r-process from a nuclear physics point of view would be around the element tin because of the bottle neck occurring at $N=82$, beyond which many more neutrons are needed to continue the fusion.

\subsection{Discussion}

This section highlights our findings and addresses key points mentioned in the previous sections, namely, scatter and inhomogeneities, the presented abundance trends, and differences between dwarfs and giants (possibly NLTE effects).

The consistently large scatter or ISM inhomogeneity seen at metallicities below $[\mathrm{Fe} / \mathrm{H}]<-2.5$ dex is found in the majority of the abundance trends. Many of the large abundance studies have found similar large star-to-star scatters at these low metallicities (e.g. Barklem et al. 2005; Preston et al. 2006; François et al. 2007; Bonifacio et al. 2009). A NLTE study of the latter carried out by Andrievsky et al. (2009), confirmed that the scatter in Ba was so large even after applying the NLTE corrections to the abundances, that they could not assume that the ISM is homogeneous. However, the very low star-to-star scatter of $\alpha$ and iron-peak element abundances provides a counter argument to this statement (Cayrel et al. 2004; Preston et al. 2006), since these elements would suggest that the ISM is very well mixed.

Our findings seem to favour an inhomogeneous early $([\mathrm{Fe} / \mathrm{H}]<-2.5)$ ISM for the reasons that follow. Considering all these (alpha, iron-peak, and neutron-capture) abundances above $[\mathrm{Fe} / \mathrm{H}]=-2.5$, all star-to-star scatters are much smaller and the ISM seems to be well-mixed. This implies that single (or a few) nucleosynthetic events such as SNe exhibit smaller effects on the stellar abundances at higher metallicity (Ishimaru \& Wanajo 1999). However, this is not the case below -2.5 dex in metallicity, where we may be witnessing the effects of very different
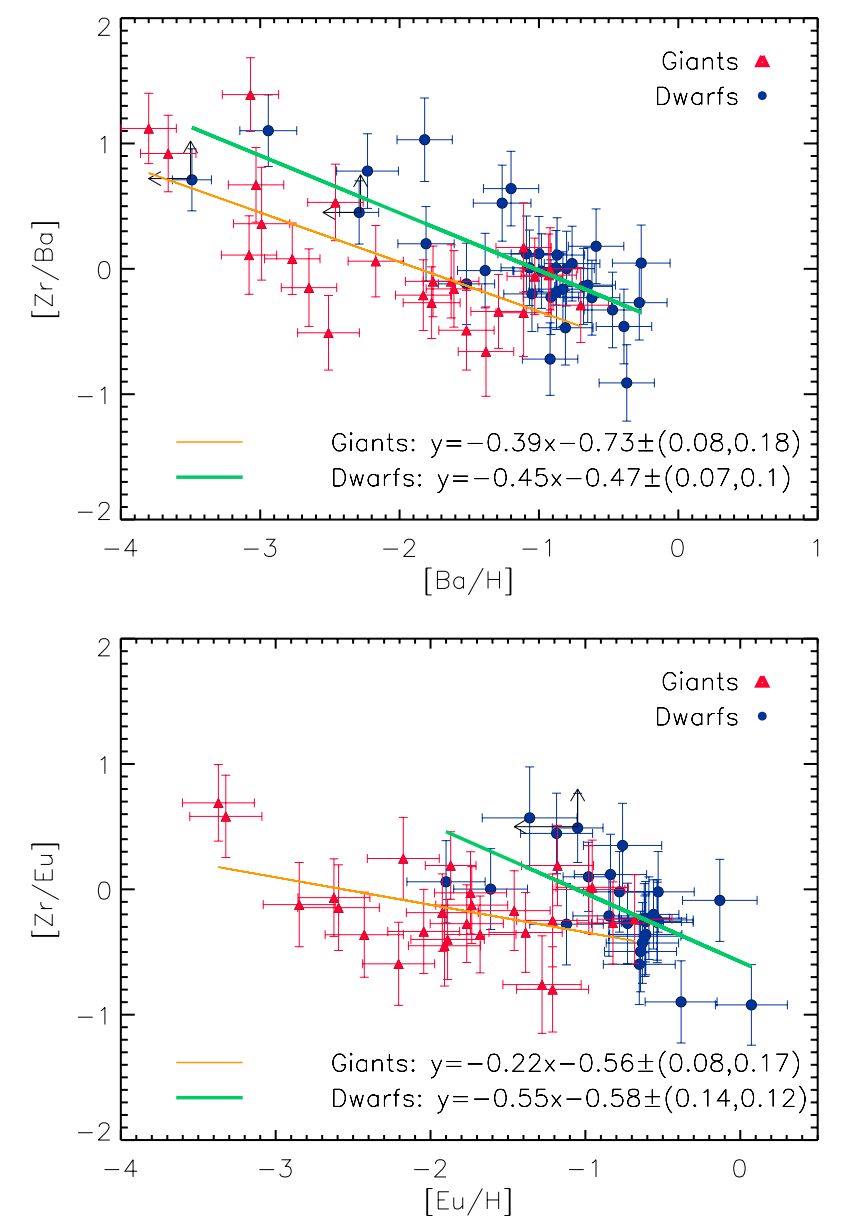

Fig. 20. Top: $[\mathrm{Zr} / \mathrm{Ba}]$ vs. $[\mathrm{Ba} / \mathrm{H}]$ showing anti-correlations. The clumping visible at higher $[\mathrm{Ba} / \mathrm{H}]$ abundances may be indicative of some common formation (s-) process for $\mathrm{Zr}$ and Ba. Bottom: $[\mathrm{Zr} / \mathrm{Eu}]$ vs. $[\mathrm{Eu} / \mathrm{H}]$, showing a clear, strong anti-correlation over the entire range of $[\mathrm{Eu} / \mathrm{H}]$ values for the dwarfs. This resembles the behaviour seen for $[\mathrm{Ag} / \mathrm{Eu}]$ vs. $[\mathrm{Eu} / \mathrm{H}]$.

(single?) exploding $\mathrm{SNe}$ (this was also suggested by Johnson \& Bolte 2002). Owing to the different supernova features their yields will vary: we refer to Heger \& Woosley (2002); Wanajo et al. (2003); Kobayashi et al. (2006); Izutani et al. (2009); Farouqi et al. (2009), and Wanajo et al. (2011) who discuss the impact that various parameters such as peak temperature, mass-cut, and entropy have on the $\mathrm{SN}$ yields. The $\alpha$-elements are mainly yielded by type II SNe and produced in one process only; they do not show this kind of scatter in their abundance pattern. The neutron-capture elements, on the contrary, seem to have several underlying formation processes, even for the same element, which may help explain the variations in the star-tostar scatters. The exact site of the neutron-capture elements is yet not known, as we have seen in the previous sections, different neutron-capture elements might be created via different channels (Johnson \& Bolte 2002; Farouqi et al. 2009). Hence, the lack of one dominating source could cause a larger scatter compared to that of the $\alpha$-elements. Furthermore, the different supernovae that create the neutron-capture elements could, due to their differing nature, lead to different neutron-capture processes, i.e. a main and a second r-process, which would help us to explain the scatter. Simply put, the inhomogeneity could in part be explained by several sources/sites yielding different amounts of the neutron-capture elements, whereas the alpha-elements are dominated by SNe II which yield relatively similar amounts of 
these elements. In contrast to the suggested range of one single r-process (Kratz et al. 2008b; Roederer et al. 2010), which is needed to explain the different abundance patterns of HD 122563 and CS22892-052, we confirm that no other group of elements be it $\alpha$, odd-Z, or Fe-peak show this kind of scatter in abundances when these originate from only one process. Furthermore, on the basis of our findings, we see that two r-processes (or primary processes) are likely needed to fully explain the correlations and the scatter.

The differences between these processes are clearly evident in Fig. 18, where the strong anti-correlation between $\mathrm{Ag}$ and $\mathrm{Eu}$, as well as that between $\mathrm{Pd}$ and $\mathrm{Eu}$, can be seen. Europium is created by the main r-process, a process that requires very high neutron number densities to produce Eu (typically around $10^{26-28} \mathrm{~cm}^{-3}$, Kratz et al. 2007), whereas the lighter isotopes of e.g. Pd can be created in environments with neutron number densities that are lower by several orders of magnitude. It is impossible to create Eu in environments with such low neutron densities (Kratz et al. 2007; Farouqi et al. 2009; Wanajo et al. 2011). This suggests that the properties of the formation sites for the heavy and the light r-process elements differ, or that the processes themselves are different. We note that Fig. 16 indicates that the process creates both $\mathrm{Ag}$ and $\mathrm{Pd}$ at almost the same rate (see also Hansen \& Primas 2011). The second r-process seems to operate effectively at all metallicities down to $[\mathrm{Fe} / \mathrm{H}]=-3.3$. This process, or its production site, must be less efficient than the main r-process. For $[\mathrm{Eu} / \mathrm{H}]>-3$, the $[\mathrm{Ag} / \mathrm{Eu}]$ is below zero and rapidly decreases with increasing Eu (see Fig. 18). However, at the lowest metallicities and europium abundances $([\mathrm{Eu} / \mathrm{H}]<-3)$ the amount of $\mathrm{Ag}$ is at the same level or slightly higher than the $\mathrm{Eu}$ abundance, as can be seen from the $[\mathrm{Ag} / \mathrm{Eu}]$ abundance being larger than zero. This could indicate that the second r-process is more efficient at low $[\mathrm{Eu} / \mathrm{H}]$. We cannot rule out that $\mathrm{Ag}$ and $\mathrm{Pd}$ both receive small contributions from the main r-process, since this process is generally $([\mathrm{Eu} / \mathrm{H}]>-3)$ predominant in the ISM gas.

Figures 13 and 17 show anti-correlations of $\mathrm{Ag}$ and $\mathrm{Pd}$ compared to $\mathrm{Sr}$ and $\mathrm{Ba}$. At high metallicities, $[\mathrm{Fe} / \mathrm{H}] \sim>-1$, the s-process is far more prevalent in the ISM than the second (weak) r-process (e.g. $[\mathrm{Ag} / \mathrm{Ba}]<0)$. However, the same figures show abundance ratios of around 0 in the range $[\mathrm{Fe} / \mathrm{H}]=-2.5$ to -1.0 . This could indicate that the s-process and the second r-process have some features or sites in common (e.g. super AGB stars), but this has yet to be confirmed.

Another important outcome of this study is the discovery of $\mathrm{Zr}$ as a "transition" element. Figures 13 to 15 show a gradual increase in the slopes of $\mathrm{Ag}$ and $\mathrm{Pd}$ compared to $\mathrm{Sr}, \mathrm{Y}$, and $\mathrm{Zr}$; i.e. an indication of the growing similarities in their formation processes. Within the uncertainties in the slopes, Ag and Pd almost correlate/show a very weak anti-correlation with Zr. When $\mathrm{Ag}$ and $\mathrm{Pd}$ are compared to each other (Fig. 16), an almost 1:1 correlation is seen. This could be the first observational evidence that at higher metallicities $([\mathrm{Fe} / \mathrm{H}]>-2.5), \mathrm{Sr}$ and $\mathrm{Y}$ are weak s-process products, as claimed by Heil et al. (2009) and Pignatari et al. (2010) (at lower metallicities Sr and Y might be created by charged particle freeze-outs; Farouqi et al. 2010; Wanajo et al. 2011). Zirconium should mainly be an s-process element (in the solar inventory), which also receives considerable contributions from a type of weak r-process. This r-process is responsible for the main production of $\mathrm{Pd}$ and $\mathrm{Ag}$. The transition from chargedparticle freeze-out or weak s- (Sr, Y) to "weak" r-process (Pd, $\mathrm{Ag})$ takes place around $\mathrm{Zr}(\mathrm{Z}=40)$, hence the name transition element. Moreover, the figures showing $[\mathrm{Ag} / \mathrm{Ba}]$ and $[\mathrm{Ag} / \mathrm{Eu}]$ yield anti-correlations (both strong, see Figs. 17, 18), suggesting that the formation processes differ. The strong anti-correlation with $\mathrm{Ba}$ shows that this process is not a main s-process and the anti-correlation with Eu demonstrates the differences between the main and the second r-process.

Finally, we turn the discussion to the differences between dwarfs and giants. Unfortunately, a full NLTE analysis is not yet available, owing to incomplete and complicated model atoms of these heavy elements. However, on the basis of previous studies of some of the heavy elements such as $\mathrm{Sr}$ and $\mathrm{Ba}$ (Belyakova \& Mashonkina 1997; Andrievsky et al. 2011, 2009), the NLTE corrections can be relatively large for low-gravity metal-poor stars. The Sr II abundance may need a correction of the order of $\sim\langle 0.2\rangle$ dex (Andrievsky et al. 2011), while the Zr II abundance corrections are lower and generally between 0.08 dex and 0.17 dex according to Velichko et al. (2010). These corrections are very dependent on the spectral line, the stellar parameters, and therefore vary from star to star. Additionally, it is insufficient to correct only one of the elemental abundances in the abundance ratios we have discussed so far. A detailed NLTE study would be needed, but is beyond the scope of this paper. Any estimate of the behaviour of the NLTE corrections of e.g. silver would be very speculative at this point, although we note from Fig. 17 that the $[\mathrm{Ag} / \mathrm{Ba}]$ ratio of the giants would need an NLTE correction of $\sim+0.5$ dex, as estimated from the offset in the figure between the giants and the dwarfs. We note that a fraction of this estimated value would be due to the NLTE correction of $\mathrm{Ba}$.

\section{A comparison to supernova yields}

To gain information on the formation site and process of our sample's abundance patterns, we compare these to two different models. The first model (model I $a+b$ ) we focus on is that of high-entropy winds (HEW) (Farouqi et al. 2009, 2010) triggered by type II SN explosions, whereas the second model (model II) is tied to low-mass electron-capture $\mathrm{SNe}$ (arising from collapsing O-Ne-Mg cores, Wanajo et al. 2011). In the last case, the neutrino interactions are taken into account.

To ensure that the abundance-to-model prediction comparison is as informative and complete as possible, we selected eight stars distributed in the following way. To probe abundance patterns that include Ag, two dwarf and six giant stars were singled out, where the giant sub-sample includes stars with special patterns such as r-rich stars. Furthermore, the selection was carried out, so that the stars cover a wide range of stellar parameters, especially metallicity. By considering stars with a wide range of $[\mathrm{Ba} / \mathrm{Eu}]$ ratios, we include stars with mixed as well as pure r-process abundance patterns (see the black diamonds in Fig. 21). The stars selected are: CD-453283 and HD 106038 (dwarfs with mixed r- and s-process patterns), BD+42621 and CS 22890-024 (giants; pure r-process tracers), HD 122563 and HD 88609 (r-poor giants), and CS 31082-001 and HD 115444 (r-rich giants). The dwarf star CD-453283 has a very high europium abundance $([\mathrm{Eu} / \mathrm{Fe}]=0.78)$, which is the highest $\mathrm{Eu}$ abundance measured for the dwarf stars in our sample. Over all, this star is overabundant in elements heavier than $\mathrm{Zr}$.

Farouqi et al. (2009, 2010) explored a large parameter space especially in entropy, where the values were varied between 20 and $275 k_{\mathrm{B}}$ /baryon, and the electron fractions, $Y_{\mathrm{e}}$, cover the range from 0.4 to 0.49 . The wind velocity adopted is $7500 \mathrm{~km} \mathrm{~s}^{-1}$. The output is neutron-to-seed ratios and corresponding yields/summed abundances. For further information we refer to Farouqi et al. (2009, 2010). Owing to the lack of wellconstrained (3D) supernova explosion parameter output, it remains unknown whether a high entropy or a low $Y_{\mathrm{e}}$ is more likely 


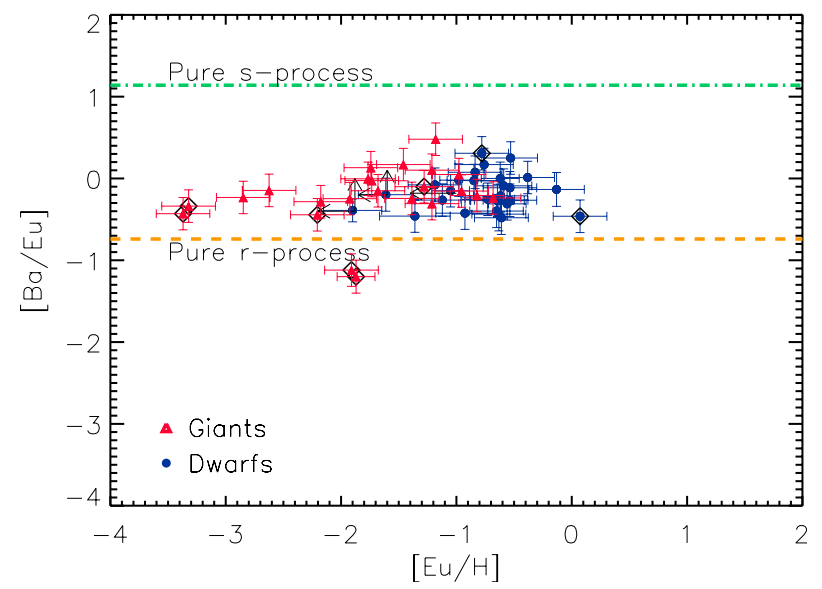

Fig. 21. Barium vs. Europium. The purity of the process is estimated based on the numbers from Arlandini et al. (1999). The black diamonds indicate the $[\mathrm{Ba} / \mathrm{Eu}]$ abundance of the eight stars shown in Figs. 25 and 27.

to happen in an actual explosion. Therefore, we carry out two different comparisons when contrasting the HEW model predictions. In model Ia) $Y_{\mathrm{e}}$ is fixed and chosen so that the value reproduces the observationally derived abundances, while the entropy, $S$, is varied. In model $\mathrm{Ib}$ ) the entropy is fixed, while $Y_{\mathrm{e}}$ is varied. The latter case enables a more direct comparison to the yield calculations of Wanajo et al. (2011).

\section{Model la}

The free parameters of the HEW models are entropy, $S \sim T^{3} / \rho$, $Y_{\mathrm{e}}$, and $V_{\text {exp }}$. All parameters are correlated and define the free neutrons per heavy seed nucleus $\left(Y_{\mathrm{n}} / Y_{\text {seed }}\right)$. Neutrino interactions have only been taken into consideration in terms of their estimated impact on the final value of $Y_{\mathrm{e}}$. The model predictions with a fixed $Y_{\mathrm{e}}$ of 0.45 are in good agreement with the derived abundances, for both the intermediate (125 $k_{\mathrm{B}}$ /baryon) and high (200 $k_{\mathrm{B}}$ /baryon) entropy (see Fig. 22). Different values of $Y_{\mathrm{e}}$ were tested in addition to 0.45 . We note that for $Y_{\mathrm{e}}=$ 0.48 , the abundances of elements heavier than $\mathrm{Zr}$ are underestimated, whereas $Y_{\mathrm{e}}=0.4$ predicts too high abundances for these elements. The estimates of 0.42 closely reproduce the observed abundances, but only the intermediate values of entropies agree well with observations - not the high entropies.

Figure 22 helps us to constrain the entropy value and/or intervals that provide enough neutron-captures to activate an r-process. Our empirical comparison to the abundances derived for HD 106038 confirms the findings of Farouqi et al. (2009), who found two entropy intervals $110<S<150$ and $150<S<$ 287 to provide enough neutrons for a weak and a main r-process, respectively. Figure 22 shows indeed that the entropies needed to create $\mathrm{Pd}$ and $\mathrm{Ag}$ are likely between 100 and $150 k_{\mathrm{B}}$ /baryon. At very high entropies $(S \sim 275)$, no lighter elements $(\mathrm{Sr}-\mathrm{Ag})$ are efficiently produced, since the fusion continues far past these elements owing to the high neutron flux.

In Fig. 25, we extend the comparison between HEW model predictions and derived stellar abundances for eight stars. For simplicity, we perform a comparison for only four entropies $\geq 125 k_{\mathrm{B}}$ /baryon. Additionally, we provide in all graphs the $[\mathrm{Fe} / \mathrm{H}]$ and the $[\mathrm{Ba} / \mathrm{Eu}]$ ratios we derived for each star. The $[\mathrm{Ba} / \mathrm{Eu}]$ ratio is especially important, because it indicates the purity of the r-process (see Fig. 21). According to Arlandini et al. (1999), $\mathrm{Ba}$ is a $81 \%$ s-process product, while $\mathrm{Eu}$ is a

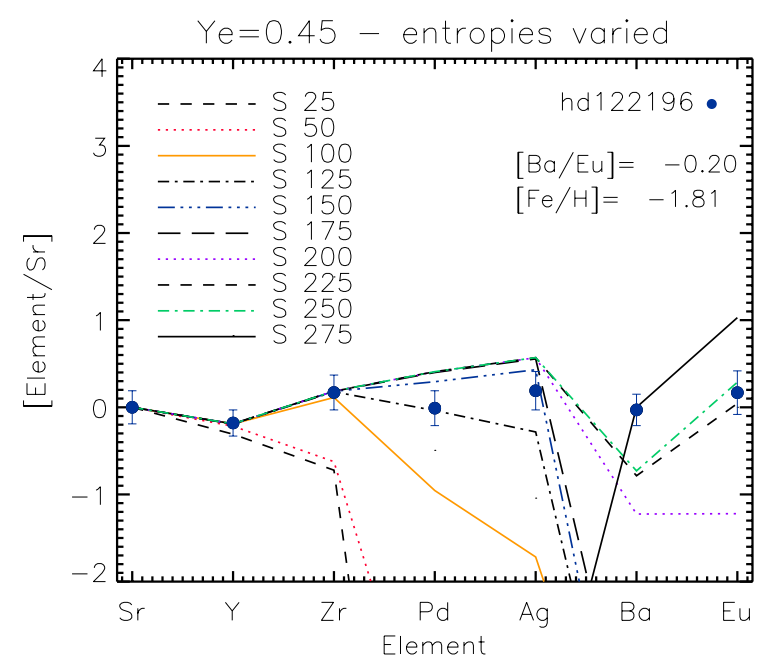

Fig. 22. Comparison of the abundances of light neutron-capture elements as derived in HD 122196 to the yields [X/Sr] produced by HEW models. The latter are computed with $Y_{\mathrm{e}}=0.45$ and varying entropies $S$ (all values listed are given in $k_{\mathrm{B}}$ /baryon units). The metallicity $([\mathrm{Fe} / \mathrm{H}])$, and $[\mathrm{Ba} / \mathrm{Eu}]$ ratio for the star are shown as well.

94\% r-process product (both in the Sun), hence, the smaller the ratio, the stronger the r-process influence is. The r-process is accepted as being pure if $[\mathrm{Ba} / \mathrm{Eu}]<-0.74$ dex Arlandini et al. (1999), which agrees with the value (-0.738) from Arndt et al. 2011. However, stars such as HD 122563 (Honda et al. 2006) provide observationally derived upper limits to this range $(\sim-0.2 \mathrm{dex})$, while a pure s-process would be found above 1.14 dex. Furthermore, the metallicity is also an important indicator of the predominant formation process, and is therefore included in the figures. Below $[\mathrm{Fe} / \mathrm{H}]=-2.5$ dex, we generally expect to see r-process yields. In Figs. 25-27 we have scaled all our derived abundances to $\mathrm{Sr}$, since we detect this element in most stars and the element is produced/predicted at most of the entropies and electron fractions considered here ${ }^{19}$. We note that plotting [element/Sr] ensures that [Sr/Sr] corresponds to zero for all lines.

Within the error bars, the observationally derived abundances agree well with the model predictions calculated with $S=125$ and $S=175 k_{\mathrm{B}} /$ baryon, although, in a few cases a model with $S=150$ would have provided closer agreement (see Fig. 25). The neutron-to-seed ratio related to these models are in the range $Y_{\mathrm{n}} / Y_{\text {seed }}=4-24 k_{\mathrm{B}} /$ baryon. From the same figure, it is furthermore evident that the heavy elements $(\mathrm{Ba}, \mathrm{Eu})$ need much higher entropies to be produced. In general, we find that the entropy interval facilitating the occurrence of the main r-process is 200-275 $k_{\mathrm{B}}$ /baryon, which is in good agreement with Farouqi et al. (2009). However, we find a slight increase in the difference between the weak $(125<S<175)$ and the main $(200<S<275)$ r-process.

Additionally, these two different processes must be r-processes since they are observed in very metal-poor stars and show patterns similar to those in the pure r-process stars.

\section{Model lb}

If we now vary the electron fractions, $Y_{\mathrm{e}}$, in the HEW model predictions, allowing these to run from $S=2 k_{\mathrm{B}} /$ baryon to a differing final entropy, we see as shown in Fig. 23 that the

${ }_{19}$ Unfortunately, $\mathrm{Fe}$ is not predicted in these models. 


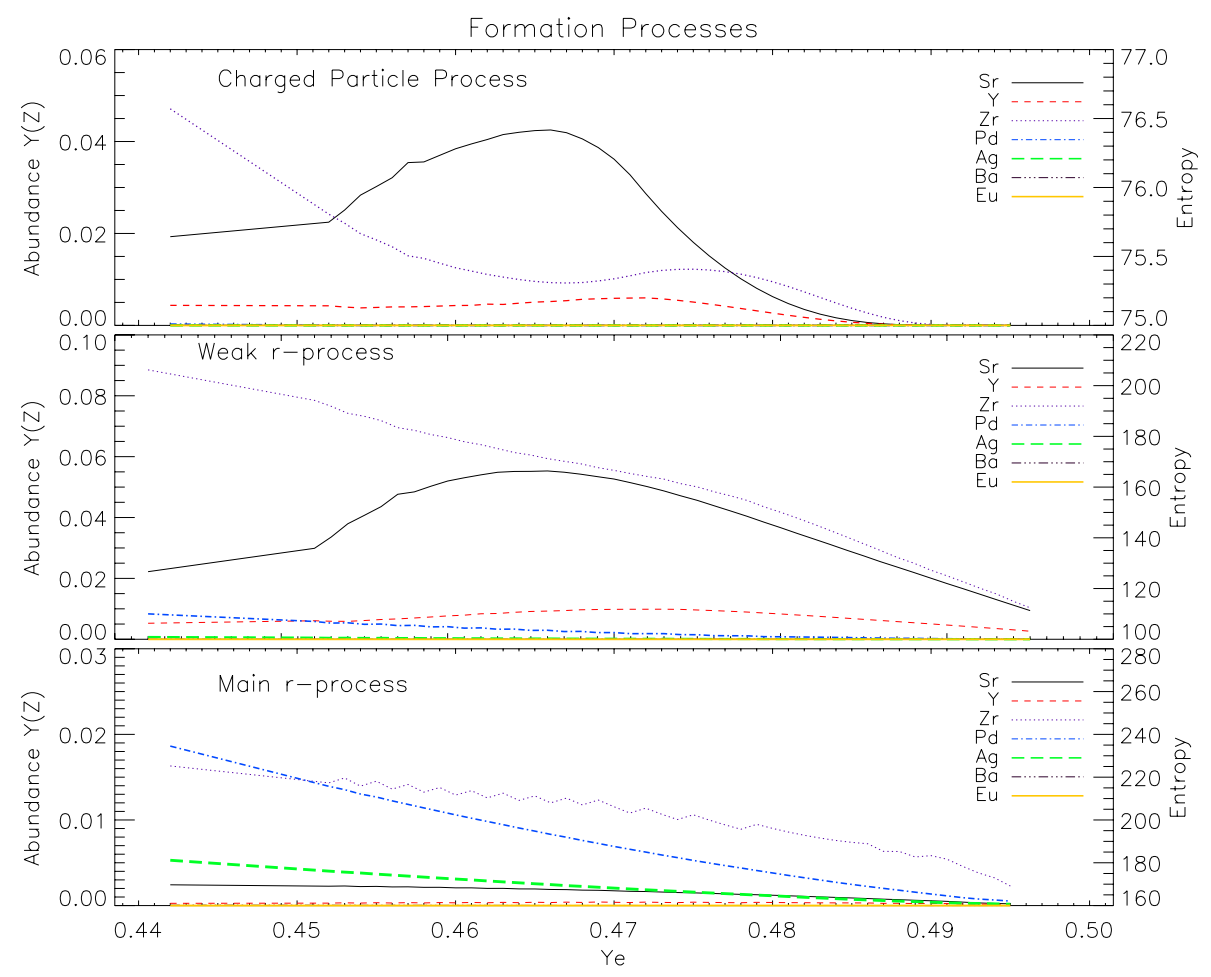

Fig. 23. HEW abundance, $Y(Z)$, predictions as a function of $Y_{\mathrm{e}}$ for the three different processes. These predicted abundances are the accumulated abundances summed over entropy for a given nucleus (see Farouqi et al. 2010, for further description). Note that the $y$-axis on the right-side of all three panels indicate the entropy intervals for each of the three processes. Every element is identified by a different colour - see legend. charged particle freeze-out, both the weak and main r-processes relate to different entropy ranges in the following way: $50<$ $S<100$ corresponds to a charged particle freeze-out for a $Y_{\mathrm{e}}$ of 0.45 , or generally speaking, this process takes place when the neutron-to-seed ratio is less than one. For the representation of this process, we adopted the mid-range value $S=75 k_{\mathrm{B}} /$ baryon - a value that always falls below a neutron-to-seed ratio of one. The weak r-process exists at $125<S<175$ (for $Y_{\mathrm{e}}=0.45$ ) or in general in the neutron-to-seed ratio from 1 to 15 (where 15 is reached at $S=155$ ), the predictions shown in Fig. 23 are made with a neutron-to-seed ratio of 5. Finally, the main r-process operates when the neutron-to-seed ratio is above 16 , and here we have shown a ratio of 30 as the representation of the main r-process (for a $Y_{\mathrm{e}}=0.45$, this corresponds to an entropy of $\sim 200-$ similar to what we found in the previous section). The yields in percentage for two different electron fractions can be found in Table 3.

In the HEW predictions with $Y_{\mathrm{e}}=0.45$ and low entropy $(S<$ 50 ), mainly iron group elements are produced owing to a very low neutron-capture efficiency at these low entropies. Therefore, we disregard this part of the entropy range to ensure that we produce and consider only heavy elements. Furthermore, not all material will necessarily be ejected in the explosion, and some fall back is to be expected.

In the uppermost panel of Fig. 23 (the charged particle plot), we see that $\mathrm{Sr}$ peaks at a $Y_{\mathrm{e}}$ of 0.47 , i.e. an environment that is not very neutron-rich, whereas the $\mathrm{Zr}$ yield rapidly increases in a more neutron-rich environment and receives contributions from both the charged particle process, the weak r-process, and a smaller contribution from the main r-process (note the different $y$-axes in Fig. 23). This was also seen in Sect. 6. However, the contribution from the main r-process was too small to be detected in the abundances. Palladium is according to the HEW predictions created by both the weak and the main r-process, but as for $\mathrm{Zr}$ the contribution from the main r-process is difficult to see in the observationally derived abundances (from which we found weak anti-correlations between $\mathrm{Pd}$ and $\mathrm{Eu}$ and $\mathrm{Zr}$ and
Table 3. Percentage of each element created, according to the HEW predictions by three different processes.

\begin{tabular}{lccc}
\hline \hline Element & $\begin{array}{c}\text { Ch. part. } \\
Y_{\mathrm{n}} / Y_{\text {seed }}<1\end{array}$ & $\begin{array}{c}\text { Weak } \mathrm{r} \\
1<Y_{\mathrm{n}} / Y_{\text {seed }}<15\end{array}$ & $\begin{array}{c}\text { Main } \mathrm{r} \\
Y_{\mathrm{n}} / Y_{\text {seed }}>15\end{array}$ \\
\hline$Y_{\mathrm{e}}=0.442$ & & & \\
$\mathrm{Sr}$ & 97.6 & 2.2 & 0.1 \\
$\mathrm{Y}$ & 96.7 & 3.3 & 0.3 \\
$\mathrm{Zr}$ & 81.6 & 18.2 & 0.2 \\
$\mathrm{Pd}$ & 4.6 & 85.3 & 10.2 \\
$\mathrm{Ag}$ & 0.7 & 82.5 & 16.8 \\
$\mathrm{Ba}$ & 0 & 0 & 100 \\
$\mathrm{Eu}$ & 0 & 0 & 100 \\
$Y_{\mathrm{e}}=0.493$ & & & \\
$\mathrm{Sr}$ & 97.6 & 2.3 & 0.1 \\
$\mathrm{Y}$ & 94.4 & 5.5 & 0.1 \\
$\mathrm{Zr}$ & 72.2 & 27.6 & 0.2 \\
$\mathrm{Pd}$ & 0.4 & 72.1 & 27.5 \\
$\mathrm{Ag}$ & 0 & 63.4 & 36.6 \\
$\mathrm{Ba}$ & 0 & 1.7 & 98.3 \\
$\mathrm{Eu}$ & 0 & 0 & 100 \\
\hline
\end{tabular}

Notes. The listed processes are: charged particle freeze-out process (Ch. part.), weak r-process (weak r), and main r-process (main r). These fractional yields (abundances) are functions of the electron fractions, $Y_{\mathrm{e}}$.

Eu). Silver needs considerably more neutron-enriched environments than $\mathrm{Zr}$ and $\mathrm{Pd}$, which we see from the slowly increasing slope, with decreasing $Y_{\mathrm{e}}$, in the main r-process plot. The heavy elements ( $\mathrm{Ba}$ and $\mathrm{Eu}$ ) need even more neutrons (lower $Y_{\mathrm{e}}$ 's) than $\mathrm{Pd}$ and $\mathrm{Ag}$ to be produced. Comparing Model $1 \mathrm{~b}$ yields to r-rich and r-poor stars (CS 31082-001 and HD 122563, respectively), we see the increasing need for neutrons with growing atomic mass (see Fig. 24). In the case of $Y_{\mathrm{e}}=0.49$, the lighter elements can be correctly reproduced by a charged particle freeze-out or a weak r-process, although, $\mathrm{Ba}$ and Eu require a main r-process entropy to be modelled in HD 122563. The 

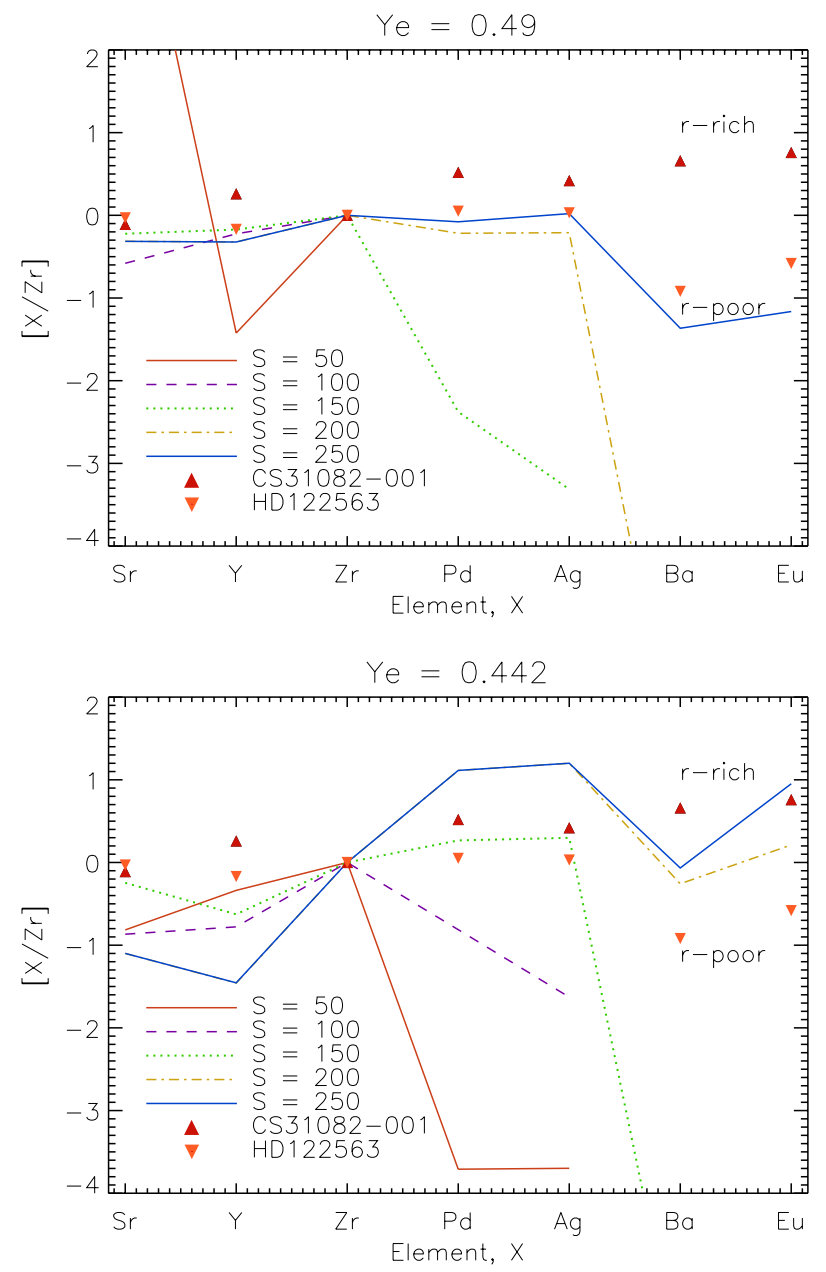

Fig. 24. HEW predictions with a neutron-poor environment $\left(Y_{\mathrm{e}}=0.49\right.$; upper plot) and a neutron-rich environment $\left(Y_{\mathrm{e}}=0.442\right.$; lower plot) compared to the r-poor star HD 122563 and r-rich star CS 31082-001.

environment is overall too neutron-poor (or limited to medium entropy) to describe the abundances of a r-rich star (see Fig. 24). In the $Y_{\mathrm{e}}=0.442$ case, the lighter to intermediate mass elements are within the $0.2-0.25$ dex uncertainties correctly reproduced by a weak r-process in both stars, whereas $\mathrm{Ba}$ and $\mathrm{Eu}$ are seen to need a main r-process and possibly an even larger neutron-toseed ratio/lower $Y_{\mathrm{e}}$ to be correctly reproduced. The need for two different processes at work is again expressed by these models and r-poor/r-rich stellar abundance patterns. The weak r-process cannot create $\mathrm{Ba}$ and $\mathrm{Eu}$ and the main r-process overproduces the intermediate elements ( $\mathrm{Pd}$ and $\mathrm{Ag}$ ). Moreover, it is also unable to correctly account for the lighter elements $(\mathrm{Sr}-\mathrm{Zr})$, where a charged particle freeze-out is needed.

\section{Model II}

The second type of model, which is connected to the lowmass $\mathrm{SN}$ of a collapsing $\mathrm{O}-\mathrm{Ne}-\mathrm{Mg}$ core are the two-dimensional (2D) models of Wanajo et al. (2011). Neutrino interaction is included in the model, and the explosion is obtained selfconsistently without any free parameters. The yields are calculated using post-processing networks, in which the output quantities from the supernova explosion, such as temperature, density, and $Y_{\mathrm{e}}$ are applied as inputs without free parameters in contrast to the parametrised HEW models. Typical input values are entropy, $S \sim 10-20 k_{\mathrm{B}} /$ baryon (much lower than in the HEW predictions), and $Y_{\mathrm{e}} \sim 0.4$ to 0.5 . The scenario allows neutron-captures to take place in the neutron-rich clumps of matter, which are convectively transported to the outer layers. Wanajo et al. (2011) found that there is little production of elements heavier than $\mathrm{Zr}$ in these conditions but suggest a possible reduction in the minimal electron fraction $Y_{\mathrm{e}, \mathrm{min}}$ below their original value of $Y_{\mathrm{e}, \mathrm{org}}=0.4$ (because of the limitation in the resolution and the two-dimensionality of the model). The impact that lower $Y_{\mathrm{e}, \min }$ values have on the yields is explored in Wanajo et al. (2011). To test this, as in their work, the additional amount of neutron-capture elements created in the neutron-rich clumps with an artificially reduced $Y_{\mathrm{e}, \text { min }}$ value is added to their original yields

$Y_{Z}\left(Y_{\mathrm{e}, \min }\right)=\frac{Y_{Z}\left(Y_{\mathrm{e}, \mathrm{org}}\right) M+\Sigma_{i} Y_{Z}(i) \Delta M_{i}}{M+\Sigma_{i} \Delta M_{i}}$,

where $\Delta M_{i}$ is the relative mass of the elements to be ejected at an $Y_{\mathrm{e}}$ lower than $Y_{\mathrm{e}, \mathrm{org}}=0.4$, and this mass is set to $2 \times 10^{-5} M_{\odot}$. The subscript $i$ runs over $Y_{\mathrm{e}}$ from $Y_{\mathrm{e}, \text { org }}$ down to $Y_{\mathrm{e} \text {,min }}$ in steps of $\Delta Y_{\mathrm{e}}=0.005 . M$ is the total mass of the ejecta calculated for the higher (original) $Y_{\mathrm{e}, \mathrm{org}}$, and this mass is $1.136 \times 10^{-2} M_{\odot} . Y_{Z}$ is the predicted yield (mole fraction) of the element with atomic number $Z$.

Yields calculated with lower $Y_{\mathrm{e}, \text { min }}$ were necessary in order to obtain considerable amounts of Pd.

With electron fractions as small as $Y_{\mathrm{e}, \min }=0.2$, Wanajo et al. (2011) found that the Ba and Eu abundances in HD 122563 could be correctly reproduced. These calculated yields are compared to the observationally derived abundances for one metal-poor giant in Fig. 26, in which we first constrain the $Y_{\mathrm{e}, \mathrm{min}}$ values. For simplicity, calculated yields have been plotted in steps of 0.05. From this figure, it is clear that the predictions calculated with an electron fraction of 0.395 and 0.35 fail to produce sufficiently large amounts of any element heavier than zirconium. Hence, these models are not be considered any further.

The usual seven heavy elements are seen to be created at different values of $Y_{\mathrm{e}, \mathrm{min}}$, where the heavier ones require a lower $Y_{\mathrm{e}, \mathrm{min}}$ because they need more neutrons to be produced. For the $\mathrm{Sr}$ to $\mathrm{Zr}$ abundances, the model predictions calculated with a $Y_{\mathrm{e}, \mathrm{min}}$ in the range from 0.35 to 0.395 show a good agreement (i.e. agree within the abundance uncertainty $\sim 0.15-0.3 \mathrm{dex}$ ) to several of the dwarf stars, Pd and Ag seem to start being produced in the proper amounts starting from values of 0.3 down to 0.2 (in $Y_{\mathrm{e}, \mathrm{min}}$ ). Similar element- $Y_{\mathrm{e}, \mathrm{min}}$ relations are found for the giants, though the $Y_{\mathrm{e} \text {,min }}$ values seem to be shifted slightly towards higher values (see Figs. 27 and 28). Bins of the required $Y_{\mathrm{e}, \mathrm{min}}$ values needed to create these seven heavy elements are for the sake of clarity shown in Fig. 28.

This difference in the electron fraction needed to produce Eu in dwarf and giant stars, could be due to the different behaviour of $\mathrm{Eu}$ in dwarfs and giants, or that the giants need large NLTE abundance corrections. Since Eu is heavier than $\mathrm{Ba}$, it seems unlikely that it would need fewer neutrons (larger $Y_{\mathrm{e}, \min }$ ) to form than $\mathrm{Ba}$ does. To compare the more extreme cases, we compare stars with strong r-process enhancements and/or depletions (r-rich: CS 31082-001 and HD 115444, r-poor: HD 122563 and HD 88609) to these model predictions as well as the HEW predictions. From Fig. 27, it becomes clear that the O-Ne-Mg core-collapse SNe may be the site for stars with weak enhancements as seen in both HD 122563 and HD 88609 (i.e. r-poor), although it is clear that the site is insufficiently neutron-rich to produce such large amounts of the heavy elements ( $\mathrm{Ba}$ and $\mathrm{Eu}$ ) and does not in general support the conditions needed for a main r-process. 
C. J. Hansen et al.: Silver and palladium help unveil the nature of a second r-process
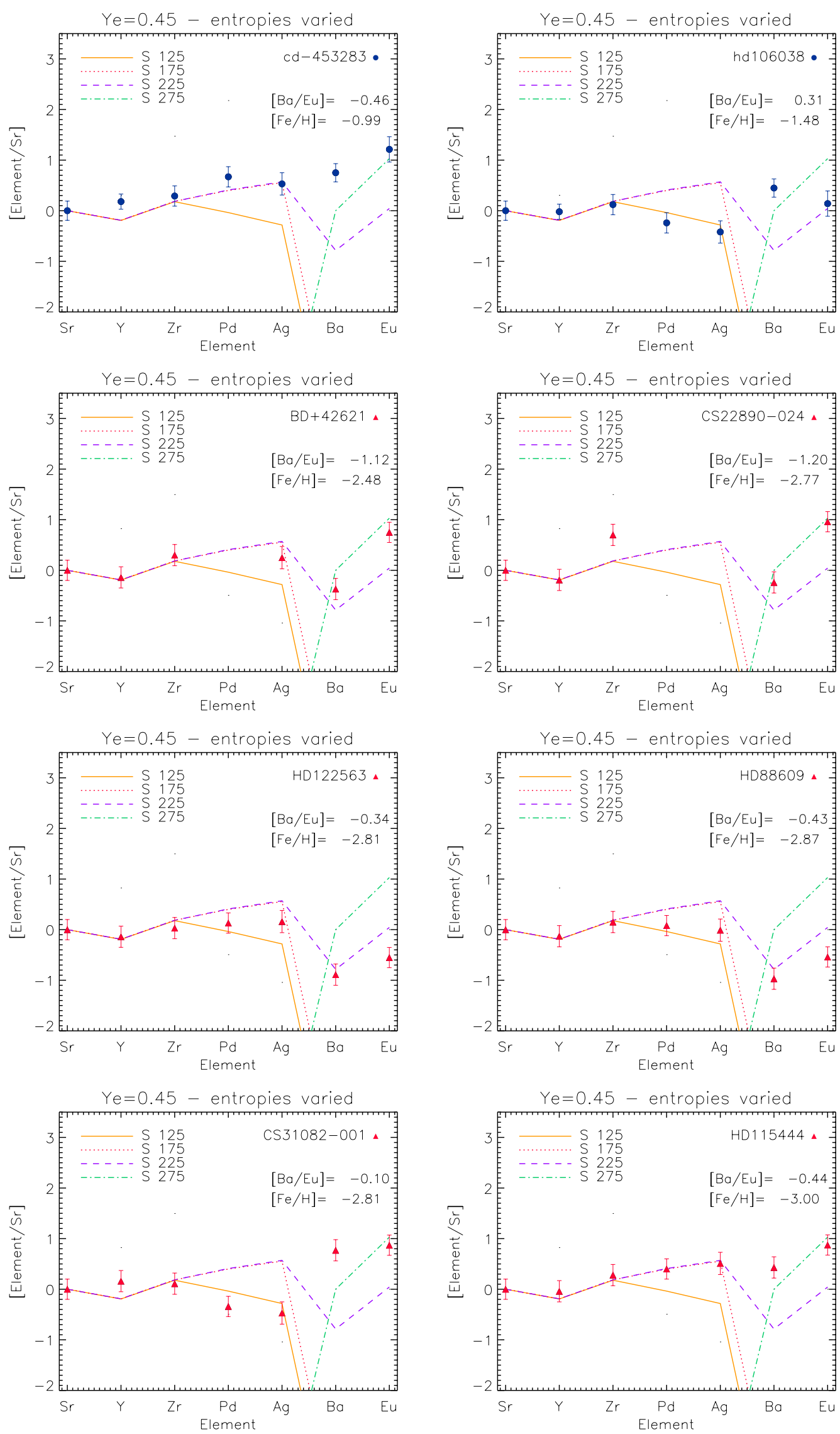

Fig. 25. HEW model yield predictions (computed assuming $Y_{\mathrm{e}}=0.45, V_{\mathrm{expan}}=7500 \mathrm{~km} \mathrm{~s}^{-1}$ and four different entropies, $S$, see legend) compared to eight different stars ( 2 dwarfs, in blue; 6 giants, in red). 


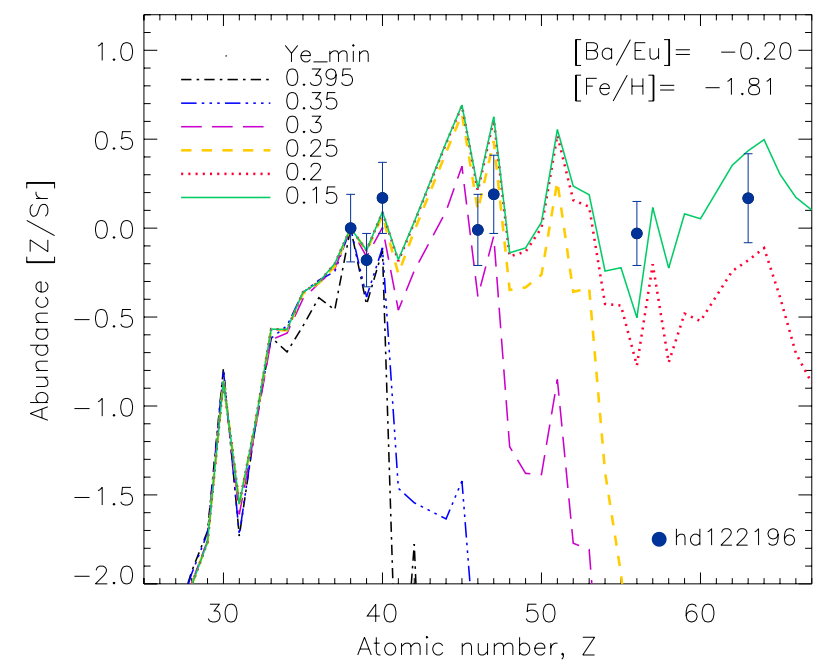

Fig. 26. HD 122196 (dwarf star) compared to different yields calculated as a function of $Y_{\mathrm{e}, \min }$. The highest electron fractions (0.395 and 0.35) are seen to have too few free neutrons to create the heavier elements $(\mathrm{Ag}-\mathrm{Eu})$.

\subsection{Discussion of yield predictions}

For the seven elements here scrutinised, both models provide satisfactory agreement with the derived abundances within their associated uncertainties in more than $60 \%$ of the stars. However, neither the HEW predictions nor the $\mathrm{O}-\mathrm{Ne}-\mathrm{Mg} \mathrm{SNe}$ model alone can provide an agreement with these seven abundances applying only one set of input parameters. Since very different entropies or electron fractions are needed, two different processes seem necessary. The faint $\mathrm{O}-\mathrm{Ne}-\mathrm{Mg} \mathrm{SNe}$ could very well be the formation site of these elements in stars such as HD 122563 and HD 88609 , i.e. stars that are relatively speaking enhanced only in the lighter elements or generally speaking r-poor. However, these supernovae are not the formation site of abundance patterns of stars such as CS 31082-001, which is also enhanced in main r-process elements such as Eu (r-rich). The entropy or neutron-richness in the ejecta from $\mathrm{O}-\mathrm{Ne}-\mathrm{Mg} \mathrm{SNe}$ are too low to facilitate a main r-process similar to that in the parametrised HEW winds explored here. Another possibility for creating the r-poor stars would be a HEW with multiple (medium+high) entropies and low electron fractions $\left(Y_{\mathrm{e}} \leq 0.442\right)$ - if possible - or a fairly high entropy and an electron fraction of 0.49 cf. Fig. 24.

Wanajo et al. (2011) showed the effect that higher dimensional models have on the predicted yields, since 1D models could not create trans-iron elements below $\mathrm{Zr}$, whereas 2D models could (in their Fig. 3). It is therefore important to consider $3 \mathrm{D}$ models before attempting to constrain the r-process site. The stellar atmospheres as well as synthesis codes may also need updates on the input physics, namely improvements in the 1D, LTE assumptions. With the current model predictions at hand, we may need to assume that there are several different sites and/or progenitors to explain the diverse abundances patterns we derive from stellar observations. On the basis of our model comparison, we cannot draw strong conclusions about the weak r-process site, but the O-Ne-Mg core-collapse SNe seem promising. However, from the abundance pattern of HD 122563 and HD 88609, the presence of Eu indicates that the ISM must have been pre-enriched by main r-process material, or that their gas was ejected from an object that allowed both the weak and main $r$-processes to coincide in the same object. For both processes the HEW winds are possible sites, since they offer the conditions needed $^{20}$, but it remains unknown whether the high entropies are physically feasible. These statements depend very much on the efficiency of the mixing in the ISM at metallicities around and below about $[\mathrm{Fe} / \mathrm{H}]=-2.5$. Therefore, we need to investigate the abundance patterns of stars in the metallicity range -3.3 to -5 dex (i.e. below the metallicities of our sample), to determine how these patterns that reflect more pristine gases behave and compare to the model predictions, before we can address in detail the mixing efficiency in the early Galaxy. Unfortunately, these abundance patterns cannot be contrasted, since we would need very high-quality spectra $(S / N>100$ at $3200 \AA)$, which would in turn require observations of very long durations for these single faint stars, in order to derive the crucial abundances, such as those of Pd and Ag.

Here as well as in previous studies, it has become evident that knowing the precise values of $Y_{\mathrm{e}}\left(Y_{\mathrm{e}, \mathrm{min}}\right)$ is essential to accurately predicting the ejected abundance patterns. The work of Winteler et al. (2012) showed that magneto-rotational corecollapse SN jets can reproduce the solar abundance pattern for $120<A<210$. Moreover, Arcones \& Montes (2011) argue that neutrino-driven winds, either proton- or neutron-rich, stemming from core-collapse $\mathrm{SNe}$ can create nuclei in the range $65<A<115$. Despite the different sites suggested, both studies illustrated the importance of knowing $Y_{\mathrm{e}}$. This in turn translates into understanding the neutrino interactions and their effect on the electron fraction (Arcones \& Montes 2011).

From the comparison of our derived abundances to the HEW model predictions, we learn that we only weakly detect process contributions of the order of $10 \%$ or less, but we need to assume that there has been more than a $10-15 \%$ contribution to see the effect of the process in our abundance ratios (cf. Table 3 ). Alternatively, our abundance ratios might actually be sensitive down to and below a process contribution of $10 \%$, since some of the estimated fractional process contributions might have been slightly overestimated. This contribution would change drastically depending on the $Y_{\mathrm{e}}$, which is affected by the other free input parameters as well as the estimated importance of neutrino interactions.

\section{Summary and conclusion}

\section{Summary}

Based on the correlations and anti-correlations of $\mathrm{Sr}, \mathrm{Y}, \mathrm{Zr}$, $\mathrm{Pd}, \mathrm{Ag}, \mathrm{Ba}$, and $\mathrm{Eu}$, it has become evident with time that the formation of the heavy elements is not a straightforward process to model, and that the previously believed solar-scaled universal r-process pattern only provides good estimates for the heaviest elements, not the elements in the atomic mass range from 38 to 47. At low metallicities $([\mathrm{Fe} / \mathrm{H}]<-2.5)$, several studies combined with this have shown that at least four different neutroncapture processes (s- and r-processes with both weak and main components) are needed to explain the observationally determined abundances. Starting with the lighter element Sr, which can in part be produced via charged particle freeze-out, this element might also receive a considerable contribution from a weak

${ }^{20}$ In their current state, the HEW model predictions allowing for a large range of parameters, especially the large span in entropies, can reproduce several different abundance patterns of all neutron-capture elements. Hence, assuming that these physical conditions are viable in one single site, they explain the patterns with one "continuous broad range r-process" (Kratz et al. 2008b; Farouqi et al. 2009; Roederer et al. 2010). 
C. J. Hansen et al.: Silver and palladium help unveil the nature of a second r-process
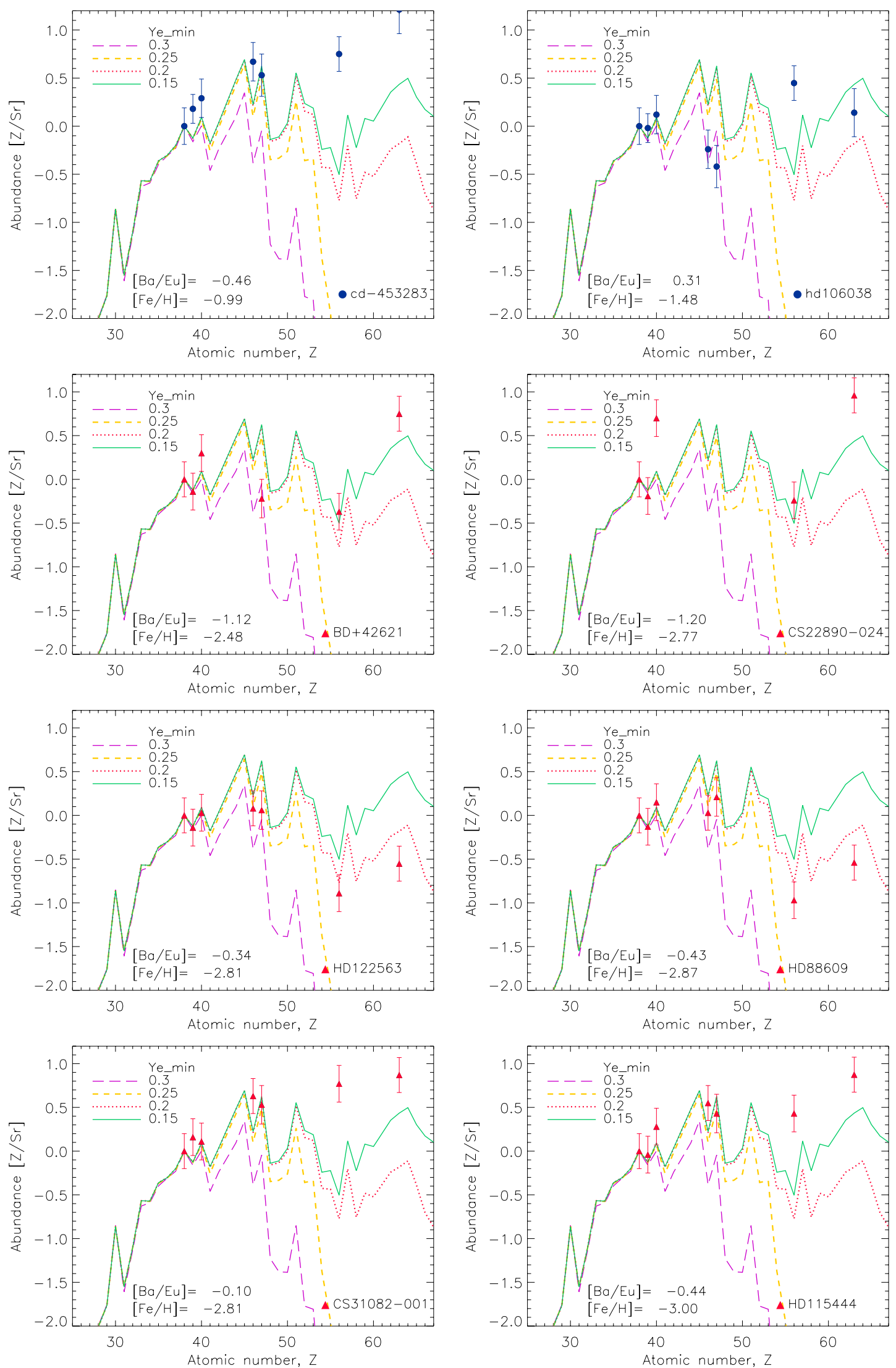

Fig. 27. O-Ne-Mg SN model yields with 0.05 step sized decreasing $Y_{\mathrm{e}, \text { min }}$ starting from $\sim 0.3$ to 0.15 compared to observationally derived abundances of dwarf stars (blue, top) and giants (red, bottom). The abundances were normalised to Sr. These model predictions fit the pattern of the r-poor star (HD 122563) better than that of the r-rich star (CS 31082-001). 
A\&A 545, A31 (2012)

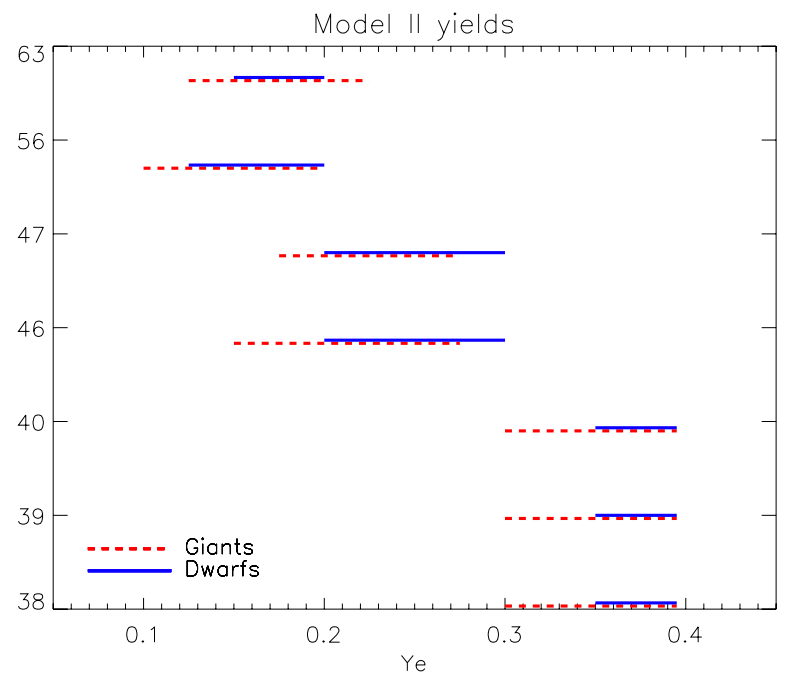

Fig. 28. Overview of the yields vs. electron fraction. Blue represents the dwarfs and red the giants. The atomic number is shown on the $y$-axis.

s-process at higher metallicities. Zirconium has shown similarities to both $\mathrm{Sr}$ and $\mathrm{Ag}$, which indicates that $\mathrm{Zr}$ is created by multiple processes, since the formations of $\mathrm{Sr}$ and $\mathrm{Ag}$ differ. Silver is created by a second/weak r-process. Its formation process clearly deviates from the main r-process responsible for forming Eu and to some extent $\mathrm{Ba}$ at low metallicity. The picture becomes even more differentiated when we try to understand the formation of $\mathrm{Ba}$. To date, an inexplicably large star-to-star scatter is found for the Ba abundances both under LTE and NLTE assumptions. No single process can explain all of these results despite the possibility that some scatter may be caused by the process occurring at various sites, in the case of the extremely metal-poor stars. Two processes might be needed to fully explain the formation of $\mathrm{Ba}$.

From the comparison to model predictions, we see that despite the different physics and parameter space investigated, the 2-3 dex star-to-star scatter in the stellar abundances cannot be explained by e.g. NLTE corrections, stellar parameter influence or sample biases, which confirms the need for at least two neutron-capture processes yielding heavy elements at very low metallicities (below -2.5 dex in $[\mathrm{Fe} / \mathrm{H}]$ ). Some scatter can arise from the different amount each site produces, and it seems necessary to have a combination of various sites to explain the individual abundance patterns that the different (r-poor vs. r-rich) stars show. One possible explanation could be that massive supernovae facilitate high-entropy winds, which create some amount of intermediate elements (in the atomic number interval range 40-50) via a weak r-process, combined with yields of the heaviest main r-process elements. According to Woosley et al. (1994), different entropies can be found within one supernova, giving rise to various entropies and processes in the exploding winds. Another site contributing to the weak r-process elements Pd and $\mathrm{Ag}$ could be the $\mathrm{O}-\mathrm{Ne}-\mathrm{Mg} \mathrm{SNe}$, which are predicted to be very common. However, according to Wanajo et al. (2011), this kind of SN cannot facilitate a main r-process, hence cannot produce $\mathrm{Eu}$, and it seems unlikely to be the dominant r-process site, which was indicated in Ishimaru et al. (2004). However, 3D models might change this picture, though it seems unlikely that these supernovae would ever reach the proper conditions to create a full main r-process. Nevertheless, we still need several sites and r-processes to explain the abundances of $\mathrm{Ag}-\mathrm{Eu}$.

\section{Conclusion}

We have found that in our observed sample of stars both dwarfs and giants show on average the same correlation/anti-correlation at all metallicities, thus we feel confident that the correlation trends combined with the large star-to-star scatter confirm the presence of two different r-processes. A second/weak r-process creating $\mathrm{Zr}-\mathrm{Ag}$ (generally elements in the atomic number range 40-50), and a main r-process producing the very heavy elements. The second r-process seems to work in intermediate entropy, and neutron number density environments, and its path possibly lies closer to stability than that of the main r-process. However, on the basis of previous studies and the current state of the model predictions, we cannot disregard the possibility that our suggested second different r-process is in fact a lower end of a continuous broad-range main r-process. Many of the physical parameters differ between the weak and the main r-process, by many orders of magnitude, and we therefore need stronger constraints on what suffices and/or is necessary to define an individual process.

In addition, it seems that several sites are needed to explain the diverse stellar abundance pattern coming from r-poor and r-rich stars. Possible formation sites are the high-entropy winds of $\mathrm{SNe}$ and neutron-rich ejecta of electron-capture $(\mathrm{O}-\mathrm{Ne}-\mathrm{Mg})$ $\mathrm{SNe}$. The yields from these objects will be mixed in the ISM, which makes tracing the original site a challenging task; furthermore, these objects are unlikely to be the only sites, and we still do not know their frequency or mixing ratios.

Acknowledgements. This work was supported by Sonderforschungsbereich SFB 881 "The Milky Way System" (subproject A5) of the German Research Foundation (DFG). The authors are grateful to the referee for comments and criticism. C.J.H. thanks ESO for the support, L. Casagrande for providing temperature estimates and C. Sneden for spectra. Furthermore, C.J.H. is very grateful to W. Walters and B. Nordström for help and discussions. F.P. acknowledges support from the Collaborative Research Project MASCHE, part of ESF EUROCORES programme EuroGENESIS. H.H. acknowledges support from the Swedish Research Council (VR) under contract 621-2011-4206. SW and C.J.H. kindly thank H.-T. Janka and B. Müller for collaboration and fruitful discussions. This research has made use of NASA's Astrophysics Data System, the SIMBAD database, operated at CDS, Strasbourg, France, and the Two Micron All Sky Survey, which is a joint project of the University of Massachusetts and the Infrared Processing and Analysis Center/California Institute of Technology, funded by the National Aeronautics and Space Administration and the National Science Foundation.

\section{References}

Alonso, A., Arribas, S., \& Martinez-Roger, C. 1994, A\&AS, 107, 365 Alonso, A., Arribas, S., \& Martinez-Roger, C. 1995, A\&A, 297, 197

Alonso, A., Arribas, S., \& Martinez-Roger, C. 1996, A\&A, 313, 873 Alonso, A., Arribas, S., \& Martínez-Roger, C. 1999, A\&AS, 140, 261 Andrievsky, S. M., Spite, M., Korotin, S. A., et al. 2009, A\&A, 494, 1083 Andrievsky, S. M., Spite, F., Korotin, S. A., et al. 2011, A\&A, 530, A105 Aoki, W., Beers, T. C., Honda, S., \& Carollo, D. 2010, ApJ, 723, L201 Arcones, A., \& Montes, F. 2011, ApJ, 731, 5

Argast, D., Samland, M., Thielemann, F., \& Qian, Y. 2004, A\&A, 416, 997 Arlandini, C., Käppeler, F., Wisshak, K., et al. 1999, ApJ, 525, 886

Arndt, O., Kratz, K.-L., Walters, W. B., et al. 2011, Phys. Rev. C, 84, 061307 Asplund, M., Grevesse, N., Sauval, A. J., \& Scott, P. 2009, ARA\&A, 47, 481 Barklem, P. S., Christlieb, N., Beers, T. C., et al. 2005, A\&A, 439, 129

Belyakova, E. V., \& Mashonkina, L. I. 1997, Astron. Rep., 41, 530

Bergemann et al. 2012, submitted

Bessell, M. S. 2005, ARA\&A, 43, 293

Bisterzo, S., Gallino, R., Straniero, O., Cristallo, S., \& Kaeppeler, F. 2011, MNRAS, 418, 284

Bonifacio, P., Monai, S., \& Beers, T. C. 2000, AJ, 120, 2065

Bonifacio, P., Spite, M., Cayrel, R., et al. 2009, A\&A, 501, 519

Burbidge, E. M., Burbidge, G. R., Fowler, W. A., \& Hoyle, F. 1957, Rev. Mod. Phys., 29, 547

Busso, M., Gallino, R., \& Wasserburg, G. J. 1999, ARA\&A, 37, 239 
Carlsson, J., Jönsson, P., \& Sturesson, L. 1990, Z. Phys. D Atoms Mol. Clusters, 16,87

Casagrande, L., Ramírez, I., Meléndez, J., Bessell, M., \& Asplund, M. 2010, A\&A, 512, A54

Cayrel, R., Depagne, E., Spite, M., et al. 2004, A\&A, 416, 1117

Christlieb, N., Beers, T. C., Barklem, P. S., et al. 2004, A\&A, 428, 1027

Cowan, R. D. 1981, The theory of atomic structure and spectra (University of California Press)

Cowan, J. J., Thielemann, F.-K., \& Truran, J. W. 1991, Phys. Rep., 208, 267

Cowan, J. J., Sneden, C., \& Truran, J. W. 2001, in AIP Conf. Ser.

Cowan, J. J., Sneden, C., Burles, S., et al. 2002, ApJ, 572, 861

Cowan, J. J., Roederer, I. U., Sneden, C., \& Lawler, J. E. 2011, Carnegie Observatories Astr. Ser., 223

Crawford, M. F., Schawlow, A. L., Gray, W. M., \& Kelly, F. M. 1949, Phys. Rev., 75, 1112

Crawford, J. L., Sneden, C., King, J. R., Boesgaard, A. M., \& Deliyannis, C. P. 1998, AJ, 116, 2489

Cutri, R. M., Skrutskie, M. F., van Dyk, S., et al. 2003, 2MASS All Sky Catalog of point sources., NASA/IPAC IRSA

Dahmen, H., \& Penselin, S. 1967, Z. Phys. 200, 456

Dekker, H., D'Odorico, S., Kaufer, A., Delabre, B., \& Kotzlowski, H. 2000, in SPIE Proc., 534

Duncan, R. C., Shapiro, S. L., \& Wasserman, I. 1986, ApJ, 309, 141

Edvardsson, B., Andersen, J., Gustafsson, B., et al. 1993, A\&A, 275, 101

Elbel, M. \& Fischer, W. 1962, Zeitschrift für Physik, 504

Farouqi, K., Kratz, K., Mashonkina, L. I., et al. 2009, ApJ, 694, L49

Farouqi, K., Kratz, K., Pfeiffer, B., et al. 2010, ApJ, 712, 1359

François, P., Depagne, E., Hill, V., et al. 2003, A\&A, 403, 1105

François, P., Depagne, E., Hill, V., et al. 2007, A\&A, 476, 935

Frebel, A., Christlieb, N., Norris, J. E., et al. 2007, ApJ, 660, L117

Freiburghaus, C., Rembges, J.-F., Rauscher, T., et al. 1999a, ApJ, 516, 381

Freiburghaus, C., Rosswog, S., \& Thielemann, F. 1999b, ApJ, 525, L121

Fröhlich, C., Martínez-Pinedo, G., Liebendörfer, M., et al. 2006, PhRvL, 96, 142502

Fuhr, J. R., Martin, G. A., \& Wiese, W. L. 1988, J. Phys. Chem. Ref. Data, 17

Fulbright, J. P. 2000, AJ, 120, 1841

Gallino, R., Bisterzo, S., Straniero, O., Ivans, I. I., \& Käppeler, F. 2006, Mem. Soc. Astron. Ital., 77, 786

Goriely, S., Bauswein, A., \& Janka, H.-T. 2011a, ApJ, 738, L32

Goriely, S., Chamel, N., Janka, H.-T., \& Pearson, J. M. 2011b, A\&A, 531, A78

Gustafsson, B., Edvardsson, B., Eriksson, K., et al. 2008, A\&A, 486, 951

Hansen, C. J., \& Primas, F. 2011, A\&A, 525, L5

Hayek, W., Wiesendahl, U., Christlieb, N., et al. 2009, A\&A, 504, 511

Heger, A., \& Woosley, S. E. 2002 (MPA Proc), 8

Heil, M., Juseviciute, A., Käppeler, F., et al. 2009, PASA, 26, 243

Hill, V., Plez, B., Cayrel, R., et al. 2002, A\&A, 387, 560

Honda, S., Aoki, W., Kajino, T., et al. 2004, ApJ, 607, 474

Honda, S., Aoki, W., Ishimaru, Y., Wanajo, S., \& Ryan, S. G. 2006, ApJ, 643, 1180

Honda, S., Aoki, W., Ishimaru, Y., \& Wanajo, S. 2007, ApJ, 666, 1189

Ishimaru, Y., \& Wanajo, S. 1999, ApJ, 511, L33

Ishimaru, Y., Wanajo, S., Aoki, W., \& Ryan, S. G. 2004, ApJ, 600, L47

Ivans, I. I., Simmerer, J., Sneden, C., et al. 2006, ApJ, 645, 613

Izutani, N., Umeda, H., \& Tominaga, N. 2009, ApJ, 692, 1517

Jackson, D. A., \& Kuhn, H. 1937, RSPSA, 158, 372

Johnson, J. A., \& Bolte, M. 2002, ApJ, 579, 616

Käppeler, F., Beer, H., \& Wisshak, K. 1989, RPPh, 52, 945

Kinman, T., \& Castelli, F. 2002, A\&A, 391, 1039

Kobayashi, C., Umeda, H., Nomoto, K., Tominaga, N., \& Ohkubo, T. 2006, ApJ, 653,1145

Korn, A. J. 2008, in ASPCS, 33

Kratz, K.-L., Bitouzet, J.-P., Thielemann, F.-K., Moeller, P., \& Pfeiffer, B. 1993, ApJ, 403, 216
Kratz, K., Farouqi, K., Pfeiffer, B., et al. 2007, ApJ, 662, 39

Kratz, K.-L., Farouqi, K., Mashonkina, L. I., \& Pfeiffer, B. 2008a, New A Rev., 52,390

Kratz, K.-L., Farouqi, K., Mashonkina, L. I., \& Pfeiffer, B. 2008b, in AIP Conf Proc., 298

Kupka, F., Ryabchikova, T. A., P. N. S. H. W. W. 2000, Baltic Astron., 9, 590

Kurucz, R. L. 1993, in (San Francisco, CA: ASP), 87

Lai, D. K., Bolte, M., Johnson, J. A., et al. 2008, ApJ, 681, 1524

Lind, K., Korn, A. J., Barklem, P. S., \& Grundahl, F. 2008, A\&A, 490, 777

Lodders, K., Palme, H., \& Gail, H.-P. 2009, in Landolt-Börnstein 4.4 (Springer), 44

Masana, E., Jordi, C., \& Ribas, I. 2006, A\&A, 450, 735

Masseron, T. 2006, Ph.D. Thesis, Observatoire de Paris

McWilliam, A. 1998, AJ, 115, 1640

Meyer, B. S. 1993, JPhG, 19, 197

Montes, F., Beers, T. C., Cowan, J., et al. 2007, ApJ, 671, 1685

Moore, C. E., Minnaert, M. G. J., \& Houtgast, J. 1966, The solar spectrum 2935 A to 8770 A (National Bureau of Standards)

Nissen, P. E., \& Schuster, W. J. 1997, A\&A, 326, 751

Nissen, P. E., Hoeg, E., \& Schuster, W. J. 1997, in Hipparcos - Venice '97, (ESA SP), 402, 225

Nissen, P. E., Primas, F., Asplund, M., \& Lambert, D. L. 2002, A\&A, 390, 235

Nissen, P. E., Chen, Y. Q., Asplund, M., \& Pettini, M. 2004, A\&A, 415, 993

Nissen, P. E., Akerman, C., Asplund, M., et al. 2007, A\&A, 469, 319

Önehag, A., Gustafsson, B., Eriksson, K., \& Edvardsson, B. 2009, A\&A, 498, 527

Ott, U., \& Kratz, K.-L. 2008, New A Rev., 52, 396

Perryman, M. A. C., Lindegren, L., Kovalevsky, J., et al. 1997, A\&A, 323, L49

Pickering, J. C., \& Zilio, V. 2001, EPJD, 13, 181

Pignatari, M., Gallino, R., Heil, M., et al. 2010, ApJ, 710, 1557

Prantzos, N., Hashimoto, M., \& Nomoto, K. 1990, A\&A, 234, 211

Preston, G. W., Sneden, C., Thompson, I. B., Shectman, S. A., \& Burley, G. S. 2006, AJ, 132, 85

Primas, F. 2010, in IAU Symp. (Cambridge Journals), 268, 221

Qian, Y.-Z., \& Wasserburg, G. J. 2001, ApJ, 559, 925

Ramírez, I., \& Meléndez, J. 2005, ApJ, 626, 465

Reddy, B. E., Lambert, D. L., \& Allende Prieto, C. 2006, MNRAS, 367, 1329

Roederer, I. U. 2009, AJ, 137, 272

Roederer, I. U., Cowan, J. J., Karakas, A. I., et al. 2010, ApJ, 724, 975

Ross, J. E., \& Aller, L. H. 1972, Sol. Phys., 25, 30

Salaris, M., Groenewegen, M. A. T., \& Weiss, A. 2000, A\&A, 355, 299

Schlegel, D. J., Finkbeiner, D. P., \& Davis, M. 1998, ApJ, 500, 525

Sneden, C. A. 1973, Ph.D. Thesis, The University of Texas at Austin

Sneden, C., Cowan, J. J., Ivans, I. I., et al. 2000, ApJ, 533, L139

Sneden, C., Cowan, J. J., Lawler, J. E., et al. 2003, ApJ, 591, 936

Sneden, C., Cowan, J. J., \& Gallino, R. 2008, ARA\&A, 46, 241

Stephens, A., \& Boesgaard, A. M. 2002, AJ, 123, 1647

Sumiyoshi, K., Terasawa, M., Mathews, G. J., et al. 2001, ApJ, 562, 880

Takahashi, K., Witti, J., \& Janka, H.-T. 1994, A\&A, 286, 857

Travaglio, C., Gallino, R., Arnone, E., et al. 2004, ApJ, 601, 864

Velichko, A. B., Mashonkina, L. I., \& Nilsson, H. 2010, Astron. Lett., 36, 664

Vogt, S. S., Allen, S. L., Bigelow, B. C., et al. 1994, in Proc. SPIE, 2198, 362

Wanajo, S., \& Ishimaru, Y. 2006, Nucl. Phys. A, 777, 676

Wanajo, S., \& Janka, H.-T. 2012, ApJ, 746, 180

Wanajo, S., Kajino, T., Mathews, G. J., \& Otsuki, K. 2001, ApJ, 554, 578

Wanajo, S., Tamamura, M., Itoh, N., et al. 2003, ApJ, 593, 968

Wanajo, S., Janka, H.-T., \& Kubono, S. 2011, ApJ, 729, 46

Wasserburg, G. J., \& Qian, Y. 2000, ApJ, 529, L21

Westin, J., Sneden, C., Gustafsson, B., \& Cowan, J. J. 2000, ApJ, 530, 783

Winteler, C., Käppeli, R., Perego, A., et al. 2012, ApJ, 750, L22

Woosley, S. E., Wilson, J. R., Mathews, G. J., Hoffman, R. D., \& Meyer, B. S. 1994, ApJ, 433, 229 


\section{Appendix A: Line lists}

Here we give the details of our line lists as well as our adopted solar abundances and additional useful information for all the heavy elements studied. Table A.1 provides the atomic information for both of the silver lines. These calculations were made without any assumption about the natural isotopic ratio. If individual isotopic $\mathrm{Ag}$ abundances are needed, these $\log g f$ values should be applied instead of those listed in Table 1 .

Table A.1. Line parameters for the resonance $5 \mathrm{~s}-5 \mathrm{p}$ in AgI.

\begin{tabular}{lccrcc}
\hline \hline Isotope & Lower level & Upper level & Flow-Fup $\begin{array}{c}\lambda_{\text {air }} \\
{[\AA]}\end{array}$ & $\log g f$ \\
\hline 107 & ${ }^{2} \mathrm{~S}_{1 / 2}$ & ${ }^{2} \mathrm{P}_{1 / 2}$ & $0-1$ & 3382.891 & -0.936 \\
107 & ${ }^{2} \mathrm{~S}_{1 / 2}$ & ${ }^{2} \mathrm{P}_{1 / 2}$ & $1-0$ & 3382.884 & -0.936 \\
107 & ${ }^{2} \mathrm{~S}_{1 / 2}$ & ${ }^{2} \mathrm{P}_{1 / 2}$ & $1-1$ & 3382.885 & -0.635 \\
109 & ${ }^{2} \mathrm{~S}_{1 / 2}$ & ${ }^{2} \mathrm{P}_{1 / 2}$ & $0-1$ & 3382.894 & -0.936 \\
109 & ${ }^{2} \mathrm{~S}_{1 / 2}$ & ${ }^{2} \mathrm{P}_{1 / 2}$ & $1-0$ & 3382.886 & -0.936 \\
109 & ${ }^{2} \mathrm{~S}_{1 / 2}$ & ${ }^{2} \mathrm{P}_{1 / 2}$ & $1-1$ & 3382.887 & -0.635 \\
107 & ${ }^{2} \mathrm{~S}_{1 / 2}$ & ${ }^{2} \mathrm{P}_{3 / 2}$ & $0-1$ & 3280.684 & -0.624 \\
107 & ${ }^{2} \mathrm{~S}_{1 / 2}$ & ${ }^{2} \mathrm{P}_{3 / 2}$ & $1-1$ & 3280.678 & -0.925 \\
107 & ${ }^{2} \mathrm{~S}_{1 / 2}$ & ${ }^{2} \mathrm{P}_{3 / 2}$ & $1-2$ & 3280.678 & -0.226 \\
109 & ${ }^{2} \mathrm{~S}_{1 / 2}$ & ${ }^{2} \mathrm{P}_{3 / 2}$ & $0-1$ & 3280.686 & -0.624 \\
109 & ${ }^{2} \mathrm{~S}_{1 / 2}$ & ${ }^{2} \mathrm{P}_{3 / 2}$ & $1-1$ & 3280.679 & -0.925 \\
109 & ${ }^{2} \mathrm{~S}_{1 / 2}$ & ${ }^{2} \mathrm{P}_{3 / 2}$ & $1-2$ & 3280.680 & -0.226 \\
\hline
\end{tabular}

Table A.2. Element and adopted solar abundances.

\begin{tabular}{cc}
\hline \hline Element & $\log \epsilon$ \\
\hline $\mathrm{Sr}$ & 2.87 \\
$\mathrm{Y}$ & 2.21 \\
$\mathrm{Zr}$ & 2.58 \\
$\mathrm{Pd}$ & 1.57 \\
$\mathrm{Ag}$ & 0.94 \\
$\mathrm{Ba}$ & 2.18 \\
$\mathrm{Eu}$ & 0.52 \\
\hline
\end{tabular}

Below we list the solar abundances that we used. These were adopted from Asplund et al. (2009).

We provide the atomic data used for the heavy elements in our line list. The values are taken from VALD (Kupka 2000). The molecular information in our line list was taken from Kurucz's home page and Kurucz (1993).
Table A.3. Atomic data for the strontium to europium: Wavelength, excitation potential, and $\log g f$.

\begin{tabular}{|c|c|c|}
\hline Sr II & $\begin{array}{c}\chi \\
{[\mathrm{eV}]}\end{array}$ & $\begin{array}{r}\log g f \\
{[\mathrm{dex}]}\end{array}$ \\
\hline 3464.45 & 3.04 & 0.49 \\
\hline 4077.71 & 0.00 & 0.17 \\
\hline 4161.79 & 2.94 & -0.50 \\
\hline 4215.52 & 0.00 & -0.14 \\
\hline Y II & $\begin{array}{c}\chi \\
{[\mathrm{eV}]}\end{array}$ & $\begin{array}{r}\log g f \\
\text { [dex] }\end{array}$ \\
\hline 3549.01 & 0.13 & -0.28 \\
\hline 3600.74 & 0.18 & 0.28 \\
\hline 3628.70 & 0.13 & -0.71 \\
\hline 3774.34 & 0.13 & 0.21 \\
\hline 3788.70 & 0.10 & -0.07 \\
\hline 3950.36 & 0.10 & -0.49 \\
\hline 4398.01 & 0.13 & -1.00 \\
\hline 4854.87 & 0.99 & -0.38 \\
\hline 4883.69 & 1.08 & 0.07 \\
\hline 5087.42 & 1.08 & -0.17 \\
\hline 5200.42 & 0.99 & -0.57 \\
\hline $\mathrm{Zr}$ II & $\begin{array}{c}\chi \\
{[\mathrm{eV}]}\end{array}$ & $\begin{array}{r}\log g f \\
\text { [dex] }\end{array}$ \\
\hline 3356.09 & 0.09 & -0.51 \\
\hline 3499.57 & 0.41 & -0.81 \\
\hline 3551.96 & 0.09 & -0.31 \\
\hline 3573.06 & 0.32 & -1.04 \\
\hline 3607.38 & 1.24 & -0.64 \\
\hline 3714.79 & 0.53 & -0.93 \\
\hline 4050.33 & 0.71 & -1.00 \\
\hline 4161.21 & 0.71 & -0.72 \\
\hline 4208.98 & 0.71 & -0.46 \\
\hline 4317.32 & 0.71 & -1.38 \\
\hline 5112.28 & 1.66 & -0.59 \\
\hline Pd I & $\begin{array}{c}\chi \\
{[\mathrm{eV}]}\end{array}$ & $\begin{array}{r}\log g f \\
\text { [dex] }\end{array}$ \\
\hline 3404.58 & 0.814 & 0.320 \\
\hline Ba II & $\begin{array}{c}\chi \\
{[\mathrm{eV}]}\end{array}$ & $\begin{array}{r}\log g f \\
{[\operatorname{dex}]}\end{array}$ \\
\hline 4554.03 & 0.00 & $0.17^{T}$ \\
\hline 4934.08 & 0.00 & $-0.15^{T}$ \\
\hline 5853.67 & 0.60 & $-1.01^{T}$ \\
\hline Eu II & $\begin{array}{c}\chi \\
{[\mathrm{eV}]}\end{array}$ & $\begin{array}{r}\log g f \\
\text { [dex] }\end{array}$ \\
\hline 4129.73 & 0.00 & $0.22^{T}$ \\
\hline 4205.04 & 0.00 & $0.21^{T}$ \\
\hline 6645.06 & 1.38 & $0.12^{T}$ \\
\hline
\end{tabular}

Notes. The " $\mathrm{T}$ " indicates that the value is the total $\log g f$, which for $\mathrm{Ba}$ was split according to McWilliam (1998) and for Eu according to Ivans et al. (2006). 
C. J. Hansen et al.: Silver and palladium help unveil the nature of a second r-process

Appendix B: Stellar parameters

Table B.1. Parameters needed to determine the temperature and gravity.

\begin{tabular}{|c|c|c|c|c|c|c|c|c|c|c|}
\hline Star & $V$ & $K$ & $\pi$ & $\sigma(\pi)$ & $E(B-V)$ & Mass* & $T$ & $g$ & {$[\mathrm{Fe} / \mathrm{H}]$} & $\xi$ \\
\hline BD-01 2916 & 9.31 & 8.03 & 20.20 & 16.60 & 0.00 & - & $4480^{a}$ & $1.20^{a}$ & -1.99 & 2.4 \\
\hline $\mathrm{BD}+82856$ & - & - & - & - & 0.00 & - & $4600^{a}$ & $0.80^{a}$ & -2.09 & 2.0 \\
\hline $\mathrm{BD}+302611$ & 9.13 & 6.09 & 3.45 & 1.31 & 0.02 & - & 4238 & $0.50^{a}$ & -1.20 & 1.7 \\
\hline $\mathrm{BD}+42621$ & 10.5 & 9.76 & 16.10 & 30.50 & 0.00 & - & $4725^{a}$ & $1.50^{a}$ & -2.48 & 1.7 \\
\hline $\mathrm{BD}+541323$ & 9.34 & 7.37 & 1.22 & 1.20 & 0.01 & - & 5213 & $2.01^{c}$ & -1.64 & 1.5 \\
\hline CS22890-024 & 13.41 & 11.44 & - & - & 0.05 & - & 5400 & $2.65^{a}$ & -2.77 & 1.7 \\
\hline CS29512-073 & 13.92 & 12.51 & - & - & 0.05 & - & $5000^{a}$ & $1.85^{a}$ & -2.67 & 1.1 \\
\hline CS30312-100 & 13.05 & 10.88 & - & - & 0.08 & - & 5200 & $2.35^{a}$ & -2.62 & 1.4 \\
\hline CS30312-059 & 13.14 & 10.70 & - & - & 0.12 & - & 5021 & $1.90^{a}$ & -3.06 & 1.5 \\
\hline CS31082-001 ${ }^{b}$ & 11.67 & 9.46 & - & - & 0.00 & - & 4925 & $1.51^{a}$ & -2.81 & 1.4 \\
\hline HD 74462 & 8.69 & 6.05 & 1.55 & 1.16 & 0.05 & - & 4590 & $1.84^{c}$ & -1.48 & 1.1 \\
\hline HD 83212 & 8.33 & 5.61 & 1.96 & 0.98 & 0.05 & - & 4530 & $1.21^{c}$ & -1.25 & 1.8 \\
\hline HD $88609^{b}$ & 8.59 & 6.01 & 0.63 & 1.14 & 0.01 & - & 4568 & $1.01^{c}$ & -2.87 & 1.9 \\
\hline HD 108317 & 8.03 & 6.15 & 4.53 & 1.06 & 0.01 & - & 5360 & 2.76 & -2.11 & 1.2 \\
\hline HD 110184 & 8.27 & 5.35 & 1.00 & 0.99 & 0.02 & - & $4450^{a}$ & $0.50^{c}$ & -2.40 & 2.1 \\
\hline HD $115444^{b}$ & 8.96 & 6.61 & 3.55 & 1.12 & 0.01 & - & 4785 & $1.50^{c}$ & -3.00 & 2.1 \\
\hline HD $122563^{b}$ & 6.20 & 3.73 & 3.76 & - & 0.025 & - & $4560^{a}$ & $0.90^{a}$ & -2.81 & 1.8 \\
\hline HD 122956 & 7.25 & 5.90 & 3.30 & - & 0.083 & - & 4700 & 1.51 & -1.45 & 1.2 \\
\hline HD 126238 & 7.66 & 5.34 & 3.81 & 0.95 & 0.04 & - & 4900 & 1.80 & -1.92 & 1.5 \\
\hline HD 126587 & 9.11 & 6.668 & 1.40 & 1.44 & 0.09 & - & $4700^{a}$ & $1.05^{c}$ & -3.16 & 1.7 \\
\hline HD 128279 & 7.97 & 7.07 & 5.96 & - & 0.10 & - & $5200^{a}$ & $2.20^{a}$ & -2.34 & 1.3 \\
\hline HD 165195 & 7.34 & 4.14 & 2.20 & - & 0.195 & - & $4200^{c}$ & $0.90^{c}$ & -2.10 & 2.1 \\
\hline HD $166161^{b}$ & 8.12 & 5.34 & 3.25 & 1.19 & 0.13 & - & $5250^{a}$ & $2.15^{c}$ & -1.25 & 1.9 \\
\hline HD 175305 & 7.18 & 5.06 & 6.18 & 0.56 & 0.03 & - & 5100 & 2.70 & -1.38 & 1.2 \\
\hline HD 186478 & 9.14 & 6.44 & 1.34 & 1.25 & 0.12 & - & 4730 & $1.50^{c}$ & -2.42 & 1.8 \\
\hline HD 204543 & 8.28 & 5.78 & 1.08 & 1.38 & 0.04 & - & 4700 & $0.80^{a}$ & -1.84 & 2.0 \\
\hline HE $0315+0000$ & 15.52 & 13.20 & - & - & 0.081 & - & 5200 & $2.40^{a}$ & -2.59 & 1.6 \\
\hline HE 0442-1234 & 12.91 & 9.96 & - & - & 0.133 & - & 4530 & $0.65^{a}$ & -2.32 & 1.8 \\
\hline HE 1219-0312 & 15.94 & 13.89 & - & - & 0.00 & - & 4600 & $1.05^{a}$ & -3.21 & 1.4 \\
\hline $\mathrm{BD}+092190$ & 11.15 & 9.91 & 1.04 & 2.79 & 0.0281 & 0.8 & $6450^{a}$ & 4.00 & -2.60 & 1.5 \\
\hline BD-133442 & 10.29 & 9.02 & - & - & 0.044 & 0.8 & 6450 & $4.20^{a}$ & -2.56 & 1.5 \\
\hline CD-3018140 & 9.95 & 8.66 & 7.32 & 1.56 & 0.030 & 0.75 & 6340 & 4.13 & -1.92 & 1.0 \\
\hline CD-33 3337 & 9.08 & 7.67 & 9.11 & 1.01 & -0.0155 & 0.8 & 5952 & 3.95 & -1.55 & 1.4 \\
\hline CD-45 3283 & 10.57 & 8.97 & 15.32 & 1.38 & 0.0001 & 0.8 & $5657^{c}$ & 4.97 & -0.99 & 0.8 \\
\hline CD-57 1633 & 9.53 & 8.09 & 10.68 & 0.91 & 0.0 & 0.8 & 5907 & 4.26 & -1.01 & 1.1 \\
\hline HD 3567 & 9.26 & 7.89 & 9.57 & 1.38 & -0.0028 & 0.82 & 6035 & 4.08 & -1.33 & 1.5 \\
\hline HD 19445 & 8.05 & 6.64 & 25.85 & 1.14 & -0.0014 & 0.70 & 5982 & 4.38 & -2.13 & 1.4 \\
\hline HD 22879 & 6.69 & 5.18 & 41.07 & 0.86 & -0.0056 & 0.8 & 5792 & 4.29 & -0.95 & 1.2 \\
\hline HD 25704 & 8.12 & 6.56 & 19.02 & 0.87 & -0.0211 & 0.8 & 5700 & 4.18 & -1.12 & 1.0 \\
\hline HD 63077 & 5.36 & 3.75 & 65.79 & 0.56 & -0.0225 & 0.8 & 5629 & 4.15 & -1.05 & 0.9 \\
\hline HD 63598 & 7.95 & 6.37 & 20.14 & 1.09 & 0.0 & 0.8 & 5680 & 4.16 & -0.99 & 0.9 \\
\hline HD 76932 & 5.80 & 4.36 & 46.90 & 0.97 & -0.024 & 0.85 & 5905 & 4.08 & -0.97 & 1.3 \\
\hline HD 103723 & 10.07 & 8.66 & 7.63 & 1.62 & 0.038 & 0.88 & 6128 & 4.28 & -0.85 & 1.5 \\
\hline HD 105004 & 10.31 & 8.87 & 2.68 & 4.49 & 0.038 & 0.8 & $5900^{a}$ & $4.30^{c}$ & -0.84 & 1.1 \\
\hline HD $106038^{b}$ & 10.18 & 8.76 & 9.16 & 1.50 & -0.025 & 0.70 & 5950 & 4.33 & -1.48 & 1.1 \\
\hline HD $111980^{b}$ & 8.37 & 6.77 & 12.48 & 1.38 & -0.0113 & 0.79 & 5653 & 3.90 & -1.31 & 1.2 \\
\hline HD 113679 & 9.70 & 8.11 & 6.82 & 1.32 & 0.024 & 0.96 & 5759 & 4.04 & -0.63 & 0.9 \\
\hline HD 116064 & 8.81 & 7.31 & 15.54 & 1.44 & 0.0352 & 0.8 & 5999 & 4.33 & -2.19 & 1.5 \\
\hline HD 120559 & 7.97 & 6.2 & 40.02 & 1.00 & 0.0070 & 0.8 & 5411 & 4.75 & -1.33 & 0.7 \\
\hline HD 121004 & 9.03 & 7.43 & 16.73 & 1.35 & 0.017 & 0.80 & 5711 & 4.46 & -0.73 & 0.7 \\
\hline HD 122196 & 8.73 & 7.28 & 9.77 & 1.32 & 0.032 & 0.78 & 6048 & 3.89 & -1.81 & 1.2 \\
\hline HD $126681^{b}$ & 9.30 & 7.63 & 19.16 & 1.44 & -0.0183 & 0.70 & 5532 & 4.58 & -1.28 & 0.6 \\
\hline HD 132475 & 8.56 & 6.91 & 10.85 & 1.14 & 0.058 & 0.75 & 5838 & 3.90 & -1.52 & 1.5 \\
\hline HD 140283 & 7.21 & 5.59 & 17.44 & 0.97 & 0.021 & 0.75 & 5738 & 3.73 & -2.58 & 1.3 \\
\hline HD 160617 & 8.73 & 7.31 & 8.66 & 1.25 & 0.0155 & 0.82 & 6028 & 3.79 & -1.83 & 1.3 \\
\hline HD 166913 & 8.23 & 6.92 & 16.09 & 1.04 & -0.004 & 0.73 & 6155 & 4.07 & -1.30 & 1.5 \\
\hline HD 175179 & 9.07 & 7.54 & 11.85 & 1.52 & -0.0056 & 0.80 & 5758 & 4.16 & -0.72 & 0.9 \\
\hline HD 188510 & 8.83 & 7.13 & 25.32 & 1.17 & 0.0141 & 0.68 & 5536 & 4.63 & -1.58 & 1.0 \\
\hline HD 189558 & 7.74 & 6.16 & 14.76 & 1.10 & 0.0042 & 0.76 & 5712 & 3.79 & -1.18 & 1.2 \\
\hline
\end{tabular}


Table B.1. continued.

\begin{tabular}{lcccccccccc}
\hline \hline Star & $V$ & $K$ & $\pi$ & $\sigma(\pi)$ & $E(B-V)$ & Mass & $T$ & $g$ & {$[\mathrm{Fe} / \mathrm{H}]$} & $\xi$ \\
\hline HD 195633 & 8.55 & 7.10 & 8.63 & 1.16 & 0.0253 & 1.10 & 6005 & 3.86 & -0.71 & 1.4 \\
HD 205650 & 9.05 & 7.57 & 18.61 & 1.23 & -0.007 & 0.70 & 5842 & 4.49 & -1.19 & 0.9 \\
HD 213657 & 9.66 & 8.35 & 5.68 & 1.54 & 0.0099 & 0.77 & 6208 & 3.78 & -2.01 & 1.2 \\
HD 298986 & 10.05 & 8.74 & 7.68 & 1.43 & 0.000 & 0.76 & 6144 & 4.18 & -1.48 & 1.4 \\
G005-040 & 10.76 & 9.13 & - & - & 0.0366 & 0.8 & 5766 & $4.23^{a}$ & -0.93 & 0.8 \\
G013-009 & 10.0 & 8.74 & 5.75 & 1.55 & 0.027 & 0.76 & 6416 & 3.95 & -2.27 & 1.4 \\
G020-024 & 11.13 & 9.67 & 5.42 & 2.32 & 0.118 & 0.78 & 6482 & 4.47 & -1.89 & 1.5 \\
G064-012 & 11.46 & 10.21 & 1.88 & 2.90 & 0.042 & 0.8 & 6459 & $4.31^{c}$ & -3.10 & 1.5 \\
G064-037 & 11.14 & 9.92 & 2.88 & 3.10 & 0.0127 & 0.8 & 6494 & $3.82^{c}$ & -3.17 & 1.4 \\
G088-032 & 10.78 & 9.54 & 3.07 & 2.32 & -0.0028 & 0.80 & 6327 & 3.65 & -2.50 & 1.5 \\
G088-040 & 8.93 & 7.51 & 12.15 & 1.24 & -0.0084 & 0.8 & 5929 & 4.14 & -0.90 & 1.4 \\
G183-011 & 9.86 & 8.60 & 6.47 & 7.85 & 0.0084 & 0.70 & 6309 & 3.97 & -2.12 & 1.0 \\
\hline
\end{tabular}

Notes. Top: Giants, bottom: Dwarfs. The superscripts a,b, and c indicate the following: ${ }^{(a)}$ Stars with $T_{\text {eff }}$ and $\log g$ derived from excitation potential and ionisation balance. ${ }^{(b)}$ Stars with a special r-process pattern - either r-poor or r-rich. ${ }^{(c)}$ Stellar parameters were altered in accordance with (a) owing to uncertainties in colour, dereddening, and parallax. ${ }^{(*)}$ For the giants we assume $M=1 M_{\odot}$. 
C. J. Hansen et al.: Silver and palladium help unveil the nature of a second r-process

\section{Appendix C: Abundances}

Table C.1 provides an overview of all the abundances and associated uncertainties determined for our sample's dwarf stars. Table C.2 gives the abundances and uncertainties for the giant stars in our sample.

Table C.1. Stellar abundances of Fe, Sr, Y, Zr, Pd, Ag, Ba, and Eu for dwarfs.

\begin{tabular}{|c|c|c|c|c|c|c|c|c|}
\hline Star & {$[\mathrm{Fe} / \mathrm{H}]$} & {$[\mathrm{Sr} / \mathrm{Fe}]$} & {$[\mathrm{Y} / \mathrm{Fe}]$} & {$[\mathrm{Zr} / \mathrm{Fe}]$} & {$[\mathrm{Pd} / \mathrm{Fe}]$} & {$[\mathrm{Ag} / \mathrm{Fe}]$} & {$[\mathrm{Ba} / \mathrm{Fe}]$} & {$[\mathrm{Eu} / \mathrm{Fe}]$} \\
\hline $\mathrm{BD}+092190$ & -2.60 & - & $-0.28 \pm 0.17$ & $-0.02 \pm 0.14$ & $0.72 \pm 0.27$ & - & - & - \\
\hline BD-13 3442 & -2.56 & $0.21 \pm 0.14$ & $-0.02 \pm 0.13$ & $0.44 \pm 0.18$ & - & - & - & - \\
\hline CD-30 18140 & -1.92 & $0.15 \pm 0.11$ & $0.1 \pm 0.12$ & $0.47 \pm 0.14$ & - & - & $-0.10 \pm 0.25$ & - \\
\hline CD-33 3337 & -1.55 & $0.22 \pm 0.11$ & $0.01 \pm 0.13$ & $0.27 \pm 0.17$ & $0.19 \pm 0.27$ & $0.27 \pm 0.25$ & $0.18 \pm 0.14$ & _- \\
\hline CD-45 3283 & -0.99 & $-0.15 \pm 0.30$ & $0.03 \pm 0.15$ & $0.14 \pm 0.17$ & $0.52 \pm 0.19$ & $0.38 \pm 0.25$ & $0.32 \pm 0.15$ & $0.78 \pm 0.17$ \\
\hline CD-57 1633 & -1.01 & $0.00 \pm 0.10$ & $-0.23 \pm 0.12$ & - & $0.17 \pm 0.23$ & $0.20 \pm 0.26$ & $0.13 \pm 0.14$ & $0.55 \pm 0.22$ \\
\hline HD 3567 & -1.33 & $-0.1 \pm 0.20$ & $-0.18 \pm 0.19$ & $0.27 \pm 0.17$ & $0.29 \pm 0.20$ & $0.53 \pm 0.25$ & $0.26 \pm 0.17$ & $0.70 \pm 0.18$ \\
\hline HD 19445 & -2.13 & $0.13 \pm 0.11$ & $-0.1 \pm 0.12$ & $0.29 \pm 0.15$ & $0.00 \pm 0.27$ & - & $-0.02 \pm 0.14$ & $0.37 \pm 0.23$ \\
\hline HD 22879 & -0.95 & $0.33 \pm 0.14$ & $-0.06 \pm 0.12$ & $0.19 \pm 0.17$ & $0.17 \pm 0.21$ & $0.00 \pm 0.26$ & $-0.18 \pm 0.18$ & $0.45 \pm 0.2$ \\
\hline HD 25704 & -1.12 & $0.30 \pm 0.11$ & $-0.05 \pm 0.12$ & $-0.02 \pm 0.17$ & $0.07 \pm 0.21$ & $0.09 \pm 0.26$ & $0.21 \pm 0.15$ & $0.48 \pm 0.17$ \\
\hline HD 63077 & -1.05 & $0.36 \pm 0.25$ & $0.06 \pm 0.13$ & $0.06 \pm 0.17$ & $0.00 \pm 0.22$ & $0.07 \pm 0.26$ & $0.22 \pm 0.15$ & $0.43 \pm 0.17$ \\
\hline HD 63598 & -0.990 & $0.41 \pm 0.14$ & $0.09 \pm 0.12$ & $0.20 \pm 0.22$ & $0.09 \pm 0.19$ & $-0.07 \pm 0.26$ & $0.19 \pm 0.15$ & $0.65 \pm 0.27$ \\
\hline HD 76932 & -0.97 & $<0.27$ & $-0.07 \pm 0.13$ & $0.19 \pm 0.17$ & $0.20 \pm 0.20$ & $0.25 \pm 0.25$ & $0.30 \pm 0.15$ & $0.41 \pm 0.16$ \\
\hline HD 103723 & -0.85 & $0.04 \pm 0.13$ & $-0.27 \pm 0.15$ & $0.05 \pm 0.17$ & $0.29 \pm 0.19$ & $0.21 \pm 0.25$ & $0.17 \pm 0.15$ & $0.43 \pm 0.16$ \\
\hline HD 105004 & -0.84 & $0.10 \pm 0.10$ & $-0.19 \pm 0.12$ & $0.02 \pm 0.17$ & $0.12 \pm 0.19$ & $0.01 \pm 0.27$ & $0.17 \pm 0.18$ & $0.26 \pm 0.20$ \\
\hline HD 106038 & -1.48 & $0.56 \pm 0.17$ & $0.54 \pm 0.14$ & $0.68 \pm 0.17$ & $0.32 \pm 0.21$ & $0.14 \pm 0.25$ & $0.76 \pm 0.17$ & $0.45 \pm 0.16$ \\
\hline HD 113679 & -0.63 & $<0.44$ & $0.08 \pm 0.12$ & $0.22 \pm 0.17$ & $0.14 \pm 0.19$ & $0.08 \pm 0.25$ & $0.25 \pm 0.15$ & $0.08 \pm 0.21$ \\
\hline HD 111980 & -1.32 & $0.45 \pm 0.22$ & $0.23 \pm 0.13$ & $0.44 \pm 0.17$ & $0.17 \pm 0.21$ & $0.10 \pm 0.25$ & $0.48 \pm 0.15$ & $<0.5$ \\
\hline HD 116064 & -2.17 & - & $0.00 \pm 0.13$ & $0.33 \pm 0.14$ & $0.37 \pm 0.21$ & - & $-0.36 \pm 0.17$ & - \\
\hline HD 120559 & -1.31 & $0.22 \pm 0.12$ & $0.25 \pm 0.15$ & $0.34 \pm 0.17$ & $0.44 \pm 0.21$ & $0.48 \pm 0.25$ & $0.22 \pm 0.17$ & $0.71 \pm 0.17$ \\
\hline HD 121004 & -0.73 & $0.40 \pm 0.12$ & $0.16 \pm 0.12$ & $0.51 \pm 0.17$ & $0.32 \pm 0.19$ & $0.16 \pm 0.25$ & $0.36 \pm 0.18$ & $0.50 \pm 0.19$ \\
\hline HD 122196 & -1.81 & $0.03 \pm 0.14$ & $-0.15 \pm 0.12$ & $0.20 \pm 0.16$ & $0.02 \pm 0.27$ & $<0.22$ & $0.02 \pm 0.16$ & $0.22 \pm 0.18$ \\
\hline HD 126681 & -1.28 & $0.20 \pm 0.19$ & $0.34 \pm 0.12$ & $0.56 \pm 0.17$ & $0.51 \pm 0.21$ & $0.28 \pm 0.25$ & $0.55 \pm 0.15$ & $0.47 \pm 0.17$ \\
\hline HD 132475 & -1.52 & $0.34 \pm 0.11$ & $0.17 \pm 0.15$ & $0.46 \pm 0.17$ & $0.34 \pm 0.19$ & $0.20 \pm 0.26$ & $0.40 \pm 0.15$ & $0.43 \pm 0.16$ \\
\hline HD 140283 & -2.58 & $-0.27 \pm 0.10$ & $-0.48 \pm 0.12$ & $-0.20 \pm 0.14$ & - & - & $<-0.62$ & - \\
\hline HD 160617 & -1.83 & $0.04 \pm 0.14$ & $-0.03 \pm 0.12$ & $0.19 \pm 0.21$ & $0.42 \pm 0.21$ & $<0.35$ & $0.41 \pm 0.16$ & - \\
\hline HD 166913 & -1.93 & $0.47 \pm 0.15$ & $0.39 \pm 0.14$ & $0.65 \pm 0.18$ & $0.42 \pm 0.23$ & $<0.63$ & $0.62 \pm 0.14$ & $0.61 \pm 0.16$ \\
\hline HD 175179 & -0.72 & $<1.28$ & $<0.95$ & $0.17 \pm 0.17$ & $0.12 \pm 0.20$ & $0.09 \pm 0.26$ & $0.45 \pm 0.14$ & $0.20 \pm 0.16$ \\
\hline HD 188510 & -1.58 & $-0.04 \pm 0.14$ & $-0.16 \pm 0.13$ & $0.18 \pm 0.17$ & $0.32 \pm 0.19$ & $0.19 \pm 0.26$ & $0.13 \pm 0.14$ & $0.40 \pm 0.18$ \\
\hline HD 189558 & -1.18 & $-0.70 \pm 0.14$ & $0.15 \pm 0.13$ & $0.44 \pm 0.17$ & $0.30 \pm 0.21$ & $0.16 \pm 0.25$ & $0.32 \pm 0.17$ & $0.39 \pm 0.16$ \\
\hline HD 195633 & -0.71 & $<1.11$ & $<0.66$ & $-0.14 \pm 0.17$ & $-0.13 \pm 0.20$ & $-0.03 \pm 0.28$ & $0.10 \pm 0.16$ & $<0.1$ \\
\hline HD 205650 & -1.19 & $-0.02 \pm 0.25$ & $0.05 \pm 0.13$ & $0.19 \pm 0.17$ & $0.22 \pm 0.21$ & $0.14 \pm 0.25$ & $0.20 \pm 0.14$ & $0.45 \pm 0.16$ \\
\hline HD 213657 & -2.01 & $0.04 \pm 0.11$ & $-0.05 \pm 0.12$ & $0.37 \pm 0.15$ & $0.22 \pm 0.27$ & $<0.53$ & $-0.03 \pm 0.15$ & - \\
\hline HD 298986 & -1.48 & $-0.03 \pm 0.17$ & $-0.09 \pm 0.14$ & $0.23 \pm 0.17$ & $0.32 \pm 0.19$ & $0.43 \pm 0.28$ & $0.14 \pm 0.16$ & $0.54 \pm 0.16$ \\
\hline G 01-039 & -2.27 & $0.16 \pm 0.10$ & $-0.07 \pm 0.12$ & $0.29 \pm 0.14$ & - & - & - & - \\
\hline G $05-040$ & -0.93 & $<1.29$ & $<1.09$ & $0.37 \pm 0.17$ & $0.16 \pm 0.21$ & $0.17 \pm 0.26$ & $0.35 \pm 0.14$ & $<0.5$ \\
\hline G 20-024 & -1.90 & $0.22 \pm 0.11$ & $0.17 \pm 0.12$ & $0.56 \pm 0.15$ & $0.77 \pm 0.27$ & - & $0.32 \pm 0.14$ & - \\
\hline G 64-012 & -3.10 & $-0.05 \pm 0.10$ & $0.02 \pm 0.13$ & - & - & - & $-0.35 \pm 0.16$ & - \\
\hline G 64-037 & -3.16 & $-0.06 \pm 0.10$ & $0.03 \pm 0.13$ & $0.52 \pm 0.14$ & - & - & - & - \\
\hline G 88-032 & -2.53 & - & $-0.14 \pm 0.13$ & $0.25 \pm 0.14$ & - & - & - & - \\
\hline G 88-040 & -0.89 & $0.04 \pm 0.10$ & $-0.27 \pm 0.15$ & $0.02 \pm 0.17$ & $0.12 \pm 0.19$ & $0.03 \pm 0.26$ & $0.05 \pm 0.14$ & $0.32 \pm 0.16$ \\
\hline G183-011 & -2.12 & - & $-0.24 \pm 0.12$ & $0.18 \pm 0.17$ & - & - & - & - \\
\hline
\end{tabular}

Notes. The "<" indicates that the abundance is an upper limit. 
Table C.2. Stellar abundances of $\mathrm{Fe}, \mathrm{Sr}, \mathrm{Y}, \mathrm{Zr}, \mathrm{Pd}, \mathrm{Ag}, \mathrm{Ba}$, and $\mathrm{Eu}$ for giants.

\begin{tabular}{|c|c|c|c|c|c|c|c|c|}
\hline Star & {$[\mathrm{Fe} / \mathrm{H}]$} & {$[\mathrm{Sr} / \mathrm{Fe}]$} & {$[\mathrm{Y} / \mathrm{Fe}]$} & {$[\mathrm{Zr} / \mathrm{Fe}]$} & {$[\mathrm{Pd} / \mathrm{Fe}]$} & {$[\mathrm{Ag} / \mathrm{Fe}]$} & {$[\mathrm{Ba} / \mathrm{Fe}]$} & {$[\mathrm{Eu} / \mathrm{Fe}]$} \\
\hline BD-01 2916 & -1.99 & $0.11 \pm 0.18$ & $0.03 \pm 0.20$ & $0.26 \pm 0.16$ & $0.39 \pm 0.20$ & $0.16 \pm 0.22$ & $0.36 \pm 0.15$ & $0.60 \pm 0.18$ \\
\hline BD+42 621 & -2.48 & $-0.18 \pm 0.15$ & $-0.32 \pm 0.19$ & $0.12 \pm 0.17$ & - & $-0.40 \pm 0.32$ & $-0.55 \pm 0.14$ & $0.54 \pm 0.16$ \\
\hline $\mathrm{BD}+82856$ & -2.09 & $-0.01 \pm 0.12$ & $0.04 \pm 0.15$ & $0.05 \pm 0.14$ & $0.12 \pm 0.21$ & $<0.73$ & $0.26 \pm 0.14$ & $0.41 \pm 0.16$ \\
\hline BD+30 2611 & -1.20 & $-0.09 \pm 0.26$ & $-0.35 \pm 0.18$ & $0.28 \pm 0.22$ & $-0.01 \pm 0.21$ & $-0.50 \pm 0.28$ & $0.28 \pm 0.14$ & $0.52 \pm 0.19$ \\
\hline $\mathrm{BD}+541323$ & -1.64 & $0.05 \pm 0.12$ & $-0.08 \pm 0.18$ & $0.01 \pm 0.16$ & $-0.33 \pm 0.20$ & $<-0.21$ & $0.35 \pm 0.14$ & $0.18 \pm 0.16$ \\
\hline CS22890-024 & -2.77 & $-0.06 \pm 0.18$ & $-0.25 \pm 0.16$ & $0.64 \pm 0.16$ & - & - & $-0.30 \pm 0.16$ & $<0.9$ \\
\hline CS29512-073 & -2.67 & $0.28 \pm 0.14$ & $0.09 \pm 0.15$ & $-0.07 \pm 0.16$ & - & - & - & $0.08 \pm 0.22$ \\
\hline CS30312-059 & -3.06 & $0.09 \pm 0.15$ & $-0.32 \pm 0.13$ & $0.43 \pm 0.37$ & - & - & $0.07 \pm 0.16$ & - \\
\hline CS30312-100 & -2.62 & $-0.34 \pm 0.20$ & $-0.79 \pm 0.12$ & $-0.17 \pm 0.17$ & - & - & - & $0.12 \pm 0.21$ \\
\hline CS31082-001 & -2.81 & $0.66 \pm 0.11$ & $0.82 \pm 0.16$ & $0.77 \pm 0.26$ & $1.29 \pm 0.19$ & $1.19 \pm 0.27$ & $1.43 \pm 0.14$ & $1.53 \pm 0.31$ \\
\hline HD 74462 & -1.48 & $0.06 \pm 0.10$ & $0.38 \pm 0.16$ & $0.54 \pm 0.26$ & $0.13 \pm 0.18$ & $-0.33 \pm 0.25$ & $0.37 \pm 0.14$ & $0.52 \pm 0.17$ \\
\hline HD 83212 & -1.25 & $-0.04 \pm 0.17$ & $0.21 \pm 0.22$ & $0.27 \pm 0.22$ & $-0.20 \pm 0.19$ & $-0.53 \pm 0.28$ & $0.32 \pm 0.14$ & $0.27 \pm 0.17$ \\
\hline HD 88609 & -2.87 & $0.04 \pm 0.11$ & $-0.09 \pm 0.23$ & $0.19 \pm 0.14$ & $<0.07$ & $<0.25$ & $-0.93 \pm 0.14$ & $-0.50 \pm 0.16$ \\
\hline HD 108317 & -2.11 & $-0.05 \pm 0.13$ & $-0.22 \pm 0.12$ & $0.07 \pm 0.14$ & $-0.08 \pm 0.20$ & $0.15 \pm 0.32$ & $0.34 \pm 0.17$ & $0.34 \pm 0.18$ \\
\hline HD 110184 & -2.40 & $-0.05 \pm 0.22$ & $0.15 \pm 0.14$ & $0.47 \pm 0.18$ & $0.22 \pm 0.23$ & $0.15 \pm 0.22$ & $-0.06 \pm 0.14$ & $0.22 \pm 0.16$ \\
\hline HD 115444 & -3.00 & $-0.08 \pm 0.16$ & $-0.12 \pm 0.14$ & $0.20 \pm 0.19$ & $0.47 \pm 0.20$ & $<0.35$ & $0.35 \pm 0.14$ & $0.79 \pm 0.16$ \\
\hline HD 122563 & -2.81 & $0.04 \pm 0.13$ & $-0.10 \pm 0.16$ & $0.07 \pm 0.18$ & $0.12 \pm 0.20$ & $0.10 \pm 0.25$ & $-0.85 \pm 0.14$ & $-0.51 \pm 0.16$ \\
\hline HD 122956 & -1.45 & $-0.04 \pm 0.14$ & $0.00 \pm 0.28$ & $-0.01 \pm 0.25$ & $0.32 \pm 0.20$ & $-0.28 \pm 0.22$ & $0.34 \pm 0.14$ & $0.24 \pm 0.16$ \\
\hline HD 126238 & -1.92 & $-0.09 \pm 0.14$ & $-0.27 \pm 0.12$ & $0.06 \pm 0.14$ & $0.24 \pm 0.19$ & $-0.01 \pm 0.28$ & $0.16 \pm 0.14$ & $0.19 \pm 0.16$ \\
\hline HD 126587 & -3.16 & $-0.01 \pm 0.10$ & $-0.20 \pm 0.13$ & $0.19 \pm 0.19$ & $0.42 \pm 0.26$ & $0.40 \pm 0.28$ & $0.08 \pm 0.14$ & $0.31 \pm 0.17$ \\
\hline HD 128279 & -2.34 & $-0.36 \pm 0.11$ & $-0.78 \pm 0.13$ & $-0.35 \pm 0.14$ & $-0.16 \pm 0.20$ & $-0.24 \pm 0.22$ & $-0.43 \pm 0.15$ & $-0.28 \pm 0.16$ \\
\hline HD 165195 & -2.10 & $-0.19 \pm 0.16$ & $-0.01 \pm 0.20$ & $0.09 \pm 0.20$ & $-0.32 \pm 0.20$ & $-0.60 \pm 0.25$ & $0.58 \pm 0.14$ & $0.89 \pm 0.17$ \\
\hline HD 166161 & -1.25 & $0.29 \pm 0.14$ & $0.29 \pm 0.18$ & $0.26 \pm 0.17$ & $0.00 \pm 0.19$ & $0.05 \pm 0.25$ & $0.55 \pm 0.15$ & $0.07 \pm 0.16$ \\
\hline HD 175305 & -1.38 & $0.01 \pm 0.19$ & $0.12 \pm 0.16$ & $0.29 \pm 0.18$ & $0.04 \pm 0.18$ & $0.04 \pm 0.28$ & $0.35 \pm 0.15$ & $0.56 \pm 0.16$ \\
\hline HD 186478 & -2.42 & $0.08 \pm 0.11$ & $0.01 \pm 0.12$ & $0.31 \pm 0.14$ & $0.26 \pm 0.18$ & $0.23 \pm 0.25$ & $0.25 \pm 0.14$ & $0.50 \pm 0.16$ \\
\hline HD 204543 & -1.84 & $-0.07 \pm 0.12$ & $-0.14 \pm 0.15$ & $0.07 \pm 0.18$ & $0.04 \pm 0.18$ & $0.04 \pm 0.25$ & $0.23 \pm 0.15$ & $0.10 \pm 0.17$ \\
\hline HE $0315+0000$ & -2.59 & $0.13 \pm 0.12$ & $0.08 \pm 0.15$ & $0.30 \pm 0.16$ & - & - & - & $0.70 \pm 0.21$ \\
\hline HE 0442-1234 & -2.32 & $-0.18 \pm 0.17$ & $-0.24 \pm 0.16$ & $-0.06 \pm 0.19$ & $0.12 \pm 0.30$ & - & - & $0.28 \pm 0.14$ \\
\hline HE 1219-0312 & -3.21 & $0.01 \pm 0.13$ & $-0.19 \pm 0.21$ & $0.19 \pm 0.14$ & - & - & $0.70 \pm 0.20$ & - \\
\hline
\end{tabular}

Notes. The "<" indicates that the abundance is an upper limit. 